Japan. J. Math.

Vol. 8, No. 1, 1982

\title{
On $l$-adic representations attached to automorphic forms
}

\author{
By Masami ОнтА \\ (Received January 6, 1981)
}

\section{Introduction}

Let $F$ be a totally real number field of finite degree $g$ over $\boldsymbol{Q}$, and $B$ a quaternion algebra over $F$ such that $B \otimes_{Q} R \cong M_{2}(R) \times H^{g-1}$, where $\boldsymbol{H}$ denotes the Hamilton quaternion algebra over $R$. The problem we consider in this paper is to construct $l$-adic representations of the Galois group $\operatorname{Gal}(\bar{F} / F)$ which are related to automorphic forms obtained from $B$. Before going into details, we briefly recall known results. For the space of cusp forms of weight 2 with respect to the congruence subgroup $\Gamma_{0}(N)$ of $S L_{2}(Z)$, the problem was first settled by Eichler [5], who proved that the main part of the (Hasse-Weil) zeta function of the modular curve associated with $\Gamma_{0}(N)$ coincides with the Dirichlet series obtained from the Hecke operators acting on the above space. Eichler's result saw deep generalizations in the work of Shimura. Especially in [16], Shimura proved that the main part of the zeta (or certain $L$ ) function of the so-called Shimura curve coincides with the Dirichlet series of Hecke type corresponding to a space of automorphic forms of weight 2 with respect to the groups obtained from $B$. For higher weight case, when $F=\boldsymbol{Q}$ and $B$ is a division algebra, Kuga and Shimura [8] have shown that the zeta function of a fibre variety, whose base is a modular curve obtained from $B$, can be expressed as a product of Dedekind zeta functions and Dirichlet series' corresponding to automorphic forms of higher weights. In the elliptic modular case, similar problem was studied by Ihara [6] by a different method. It was completed by Deligne [3], who first constructed $l$-adic representations attached to elliptic cusp forms of higher weights. Similar topic was studied by Langlands [10] from the representation theoretic point of view. Langlands [11] has also established the coincidence of the zeta function of a higher dimensional Shimura variety and a product of the $L$-functions attached to automorphic forms, for totally indefinite division quaternion algebras.

The author was partially supported by Sonderforschungsbereich "Theoretische Mathematik", Universität Bonn, during the preparation of this paper. 
Besides these work, there was an unpublished work of Shimura [19]. In that paper, starting from a representation $\rho$ as below, Shimura has constructed $l$-adic representations of $\mathrm{Gal}(\bar{F} / F)$ by interpreting the so-called Eichler-Shimura cohomology groups corresponding to a certain space of autmorphic forms obtained from $B$, in algebro-geometric terms. He has also established the congruence relation for the Hecke operators acting on the $l$-adic representation space he constructed. But it has remained an open problem whether that $l$-adic representation has the property (2) of the theorem below. Our work in this paper was motivated by that work, and studies the same subject as in [19], borrowing many ideas from it. In fact, a large part of this paper is a reformulation of [19]. But in constructing the $l$-adic representation, we use an idea of Langlands [10], namely we use certain étale cohomology groups which are closely related to the EichlerShimura cohomology groups.

We now explain the main result of this paper. Let $F$ and $B$ be as above, and suppose that $B$ is a divison algebra. We start with a representation $\rho$ of $B^{\times}$on $\boldsymbol{Q}^{m}$;

$$
\rho: B^{\times} \longrightarrow \mathrm{GL}_{m}(\boldsymbol{Q})
$$

which satisfies the following conditions:

( $\rho 1) \quad \rho$ is a homomorphism of algebraic groups over $\boldsymbol{Q}$.

( $\rho 2)$ There is an integer $w$ such that $\rho(c)=N_{F / Q}(c)^{w}$ for all $c \in F^{\times}$.

From such a representation $\rho$, we can define the space $\subseteq(S, \rho)$ of automorphic forms for each subgroup $S$ of $B_{A^{+}}^{\times}$which is a product of $B_{\infty+}^{\times}$and an open compact subgroup of $B_{f}^{\times}$(see Notation and terminology below for these symbols). We can define, in the usual manner, the Hecke operators $\mathfrak{I}(\mathfrak{p})$ and $\mathfrak{I}(\mathfrak{p}, \mathfrak{p})$ for finite primes $\mathfrak{p}$ of $F$ such that $\mathfrak{i}) \mathfrak{p}$ does not ramify in $B$, and ii) $S$ contains the unit group of a maximal order of $B_{p}$. Suppose that $\rho$ also satisfies the following conditions:

( $\rho 3)$ For a maximal order o of $B$, there is a $Z$-lattice $X$ in $\boldsymbol{Q}^{m}$ which has the following properties:

(

(o3.2) For any positive integer $a$ and any $\alpha \in \mathfrak{D} \cap B^{\times}$such that $\alpha-1 \in a \mathfrak{D}$, it holds that $(\rho(\alpha)-\rho(1)) X \subseteq a X$.

( $\rho 4)$ There exists a non-degenerate bilinear form (, ) on $\boldsymbol{Q}^{m}$ such that $(\rho(\alpha) x, \rho(\alpha) y)=N_{F / Q}(\nu(\alpha))^{w}(x, y)$ for all $x, y \in \boldsymbol{Q}^{m}$ and $\alpha \in B^{\times}$, where $\nu$ denotes the reduced norm.

Our main result in this paper is the following 
Theorem ((4.3.1)). Let $\rho$ be a representation satisfying the above conditions $(\rho 1)-(\rho 4)$, and suppose that $S$ is stable under the canonical involution. Then, for each rational prime $l$, there exists an l-adic representation

$$
\psi_{S, \rho}: \operatorname{Gal}(\bar{F} / F) \longrightarrow \mathrm{GL}_{2 \mathrm{dim} C} \Subset(S, \rho)\left(\boldsymbol{Q}_{l}\right),
$$

which has the following properties:

(1) For a finite prime $\mathfrak{p}$ of $F$ such that i) $p$ does not divide $l$, ii) $S$ conthe the unit group of a maximal order of $B_{\mathfrak{p}}$, and iii) p does not ramify in $B$, tains representation $\psi_{s, \rho}$ is unramified at $\mathfrak{p}$.

(2) Let $p$ be a finite prime of $F$ as in (1), and $\sigma_{\mathfrak{p}}$ a Frobenius element at $\mathfrak{p}$ in $\operatorname{Gal}(\bar{F} / F)$. Then we have

$$
\operatorname{det}\left(1-\psi_{S, \rho}\left(\sigma_{\mathfrak{p}}\right) T\right)=\operatorname{det}\left(1-\mathfrak{I}(\mathfrak{p}) T+N_{F / Q}(\mathfrak{p}) \mathfrak{T}(\mathfrak{p}, \mathfrak{p}) T^{2} \mid \mathfrak{S}(S, \rho)\right) .
$$

The content of this paper is as follows. In $\S 1$, starting from a representation $\rho$ satisfying $(\rho 1)$ and $(\rho 2)$, we define and study the spaces $\widetilde{S}(S, \rho)$ and the Hecke operators acting on them. This section is of completely expository nature, and use only routine arguments. We mainly follow the method of Shimura [15] and [18]. In § 2, we first study the Eichler-Shimura cohomology groups attached to our spaces $\subseteq(S, \rho)$, and then the (classical) sheaf cohomology groups related to them. We next interpret them in terms of the étale cohomology groups introduced by Langlands [10]. The theory of canonical models (Shimura [17]) is of course indispensable in this part. At the end of $\S 2$, we construct certain $l$-adic representation attached to $\widetilde{S}(S, \rho)$ using Langlands' étale cohomology groups under some condition on $S$. After these two expository sections, we prove in $\S 3$ the congruence relation for our $l$-adic representation ((3.8.5)), which plays the essential role in our theory. Here the key tool is Shimura's congruence relation ([17] 2.23). We also employ Morita's good reduction theorem for Shimura curves (Morita [13]). Before Morita's result, we could prove our assertions (1) and (2) above only for "almost all $\mathfrak{p}$ ". In proving the congruence relation, we use Shimura's map $\omega$, which was originally used to interpret the EichlerShimura cohomology groups into algebro-geometric terms in [19], to interpret the étale cohomology groups in an elementary language. Although it might be possible to eliminate this part by the full use of étale cohomology theory for Morita's $W_{S}$, we do not do this in this paper. We think that Shimura's theorem (3.1.3), which asserts that the kernel of $\omega$ is finite, deserves attention for its own interest. Anyway, the proof of our congruence relation is nothing more than a computation after (3.1.3). Using the results of $\S \S 1-3$, we prove our main theorem above in $\S 4$. In $\S 5$, we construct representations $\rho$ satisfying the conditions $(\rho 1)-(\rho 4)$. 
As is clear from what we have said above, this paper owes much to Shimura's work, and I would like to emphasize our indebtedness to [19]. In concluding this introduction, I would like to express my deepest gratitude to Professor G. Shimura for allowing me to see his important unpublished work, and also for his encouragement and suggestions. Also, I would like to thank Professor Y. Ihara for valuable discussions during the preparation of this paper.

Notation and Terminology. As usual, we denote by $\boldsymbol{Z}, \boldsymbol{Q}, \boldsymbol{R}$ and $\boldsymbol{C}$ the ring of rational integers, the field of rational numbers, the field of real numbers, and the field of complex numbers, respectively. For a finite algebraic number field $F$, we denote by $\mathfrak{r}_{F}$ the ring of integers in $F$. For a finite prime $\mathfrak{p}$ of $F, \mathfrak{r}_{F \mathfrak{p}}$ and $F_{\mathfrak{p}}$ mean, respectively, the $\mathfrak{p}$-completion of $\mathfrak{r}_{F}$ and $F$. For a quaternion algebra $B$ over $F$ and an order $\mathfrak{o}$ of $B$, we use similar notation $\mathfrak{o}_{\mathfrak{p}}$ and $B_{\mathfrak{p}}$ to denote the $\mathfrak{p}$-completion. The adelization of $B$ is denoted by $B_{A}$. Its finite (resp. infinite) component is denoted by $B_{f}$ (resp. $B_{\infty}$ ). We use similar notation for algebraic groups, e.g. the multiplicative group $B^{\times}$of invertible elements in $B$. We denote by $B_{\infty+}^{\times}$the connected component of the identity of $B_{\infty}^{\times}$, and put $B_{A^{+}}^{\times}=B_{\infty+}^{\times} \times B_{f}^{\times}$. For $B$ over $F$ as above, the reduced norm of $B$ over $F$ is denoted by $\nu_{B / F}$. When there is no fear of confusion, we simply write $\nu$ for it. The discriminant of $B$ over $F$ is denoted by $D(B / F)$.

For a field $k$, we denote by $\bar{k}$ an algebraic closure of $k$. For a Galois extension $K$ of $k$, we denote by $\operatorname{Gal}(K / k)$ the Galois group of $K$ over $k$. We let $\operatorname{Gal}(K / k)$ act on $K$ from the right, i.e. $x^{\sigma \tau}=\left(x^{\sigma}\right)^{\tau}$ for $x \in K$ and $\sigma, \tau \in \mathrm{Gal}$ $(K / k)$. For an algebra $R, M_{n}(R)$ denotes the ring of matrices of size $n$ with entries in $R$. The transpose of $A \in M_{n}(R)$ is denoted by ${ }^{t} A$. For a nonnegative integer $n$ and a field $k$, which depends on the context, we denote by $\rho_{n}$ the representation of $G L_{2}(k)$ on the space of symmetric tensors of degree $n$ obtained from $k^{2}$. We identify it with the representation on the space of homogeneous polynomials of degree $n$ in two variables with coefficients in $k$ as in Shimura [18] 8.2.

\section{$\S 1$. The space $\subseteq(S, \rho)$}

1.1. Let $F$ be a totally real number field of degree $g$ over $\boldsymbol{Q}$, and $B$ a division quaternion algebra over $F$ such that $B \otimes_{Q} \boldsymbol{R} \cong M_{2}(\boldsymbol{R}) \times \boldsymbol{H}^{g-1}$, where $\boldsymbol{H}$ denotes the Hamilton quaternion algebra over $\boldsymbol{R}$. We assume that the identity injection of $F$ into $R$ splits $B$. We are going to define and study the space $\widetilde{S}(S, \rho)$ of certain automorphic forms obtained from $B$ in this section. We start with a representation 


$$
\rho: B^{\times} \longrightarrow G L_{m}(\boldsymbol{Q})
$$

which satisfies the following two conditions:

(1.1.1) $\rho$ is a $\mathbf{Q}$-rational homomorphism of algebraic groups over $\boldsymbol{Q}$, if we consider $B^{\times}$as an algebraic group over $\boldsymbol{Q}$ in a natural manner (i.e. $B^{\times}(R)=$ $\left(B \otimes_{Q} R\right)^{\times}$for any $\mathbf{Q}$-algebra $\left.R\right)$.

Here and henceforth, we use the same symbol $B^{\times}$to denote the algebraic group over $\boldsymbol{Q}$, and its $\boldsymbol{Q}$-rational points. The context will always make clear its meaning.

(1.1.2) There exists an integer $w$ such that $\rho(c)=N_{F / Q}(c)^{w}$ for all $c \in F^{\times}$.

1.2. Let $\rho_{R}$ be the representation of $\left(B \otimes_{Q} R\right)^{\times}$obtained from $\rho$ by taking the $R$-valued points. By the assumption on $B,\left(B \otimes_{Q} R\right)^{\times}$is isomorphic to $G L_{2}(\boldsymbol{R}) \times\left(\boldsymbol{H}^{\times}\right)^{g-1}$. We fix such an isomorphism once for all, and identify them. Therefore we have

$$
\rho_{\boldsymbol{R}}: G L_{2}(\boldsymbol{R}) \times\left(\boldsymbol{H}^{\times}\right)^{g-1} \longrightarrow G L_{m}(\boldsymbol{R}) .
$$

Let $\rho_{R}^{+}$be the restriction of $\rho_{R}$ to $G L_{2}^{+}(R) \times\left(H^{\times}\right)^{g-1}$, and $\rho_{R}^{u}$ the restriction of $\rho_{\boldsymbol{R}}$ to $S L_{2}(\boldsymbol{R}) \times\left(\boldsymbol{H}^{u}\right)^{g-1}$, where $G L_{2}^{+}(\boldsymbol{R})=\left\{x \in G L_{2}(\boldsymbol{R}) \mid \operatorname{det}(x)>0\right\}$ and $\boldsymbol{H}^{u}=$ $\left\{x \in H^{\times} \mid \nu(x)=1\right\}, \nu$ being the reduced norm of $\boldsymbol{H}$ over $\boldsymbol{R}$. Now we have a natural decomposition:

$$
G L_{2}^{+}(\boldsymbol{R}) \times\left(\boldsymbol{H}^{\times}\right)^{g-1} \stackrel{\sim}{\longrightarrow}\left(\boldsymbol{R}_{+}^{\times}\right)^{g} \times S L_{2}(\boldsymbol{R}) \times\left(\boldsymbol{H}^{u}\right)^{g-1},
$$

which sends $\left(x_{1}, \cdots, x_{g}\right)$ to $\left(\operatorname{det}\left(x_{1}\right)^{1 / 2}, \nu\left(x_{2}\right)^{1 / 2}, \cdots, \nu\left(x_{g}\right)^{1 / 2}, \operatorname{det}\left(x_{1}\right)^{-1 / 2} x_{1}, \nu\left(x_{2}\right)^{-1 / 2} x_{2}\right.$, $\left.\cdots, \nu\left(x_{g}\right)^{-1 / 2} x_{g}\right)$, where $\boldsymbol{R}_{+}^{\times}$denotes the set of positive real numbers. By the condition (1.1.2), we have

$$
\rho_{R}\left(\left(c_{1}, \cdots, c_{g}\right)\right)=\left(\prod_{i=1}^{g} c_{i}\right)^{w} \text { for all } \quad\left(c_{1}, \cdots, c_{g}\right) \in\left(\boldsymbol{R}_{+}^{\times}\right)^{g} .
$$

On the other hand, since $\rho_{\boldsymbol{R}}^{u}$ is an $\boldsymbol{R}$-rational representation of $S L_{2}(\boldsymbol{R}) \times$ $\left(\boldsymbol{H}^{u}\right)^{g-1}$, we see that

(1.2.2) There exist non-negative integers $m_{k}(1 \leqslant k \leqslant r)$ and $R$-irreducible representations $\sigma_{k}$ of $\left(H^{u}\right)^{g-1}(1 \leqslant k \leqslant r)$, and $\rho_{R}^{u}$ is isomorphic to the direct sum $\oplus_{k=1}^{r} \rho_{m_{k}} \otimes \sigma_{k}$ over $\boldsymbol{R}$, where $\rho_{m_{k}}$ denotes the symmetric tensor representation of degree $m_{k}$ of $S L_{2}(\boldsymbol{R})$.

For each $k(1 \leqslant k \leqslant r)$, we define the representation $\Psi_{k}$ of $G L_{2}^{+}(\boldsymbol{R}) \times\left(\boldsymbol{H}^{\times}\right)^{g-1}$ by

$$
\Psi_{k}\left(\left(x_{1}, \cdots, x_{g}\right)\right)=\left(\operatorname{det}\left(x_{1}\right) \prod_{i=2}^{g} \nu\left(x_{i}\right)\right)^{w / 2} \sigma_{k}\left(\left(\nu\left(x_{2}\right)^{-1 / 2} x_{2}, \cdots, \nu\left(x_{g}\right)^{-1 / 2} x_{g}\right)\right)
$$


Then $\rho_{R}^{+}$is equivalent over $R$ to the direct sum of the representations which $\operatorname{map}\left(x_{1}, \cdots, x_{g}\right) \in G L_{2}^{+}(\boldsymbol{R}) \times\left(\boldsymbol{H}^{\times}\right)^{g-1}$ to $\operatorname{det}\left(x_{1}\right)^{-m_{k} / 2} \rho_{m_{k}}\left(x_{1}\right) \otimes \Psi_{k}\left(\left(x_{1}, \cdots, x_{g}\right)\right)$ for $1 \leqslant k \leqslant r$.

1.3. Let $B_{+}^{\times}$denote the elements of $B^{\times}$whose reduced norms are totally positive. If we denote by $p$ the composite of the natural embedding $B^{\times} \subset$ $\left(B \otimes_{Q} \boldsymbol{R}\right)^{\times}=G L_{2}(\boldsymbol{R}) \times\left(\boldsymbol{H}^{\times}\right)^{g-1}$ and the projection to $G L_{2}(\boldsymbol{R}), B_{+}^{\times}$acts on the complex upper half plane $H$ through $p$ by linear fractional transformations. Let $\Psi$ be a representation of $B_{+}^{\times}$on $\boldsymbol{R}^{d} ; \Psi: B_{+}^{\times} \rightarrow G L_{d}(\boldsymbol{R})$. For a $\boldsymbol{C}^{d}$-valued function $f(z)$ on $H, \gamma \in B_{+}^{\times}$and a natural number $n$, we put

(1.3.1) $\left(f \mid[\gamma]_{n, \Psi}\right)(z)=\operatorname{det}(p(\gamma))^{n / 2} j(p(\gamma), z)^{-n} \Psi(\gamma)^{-1} f(p(\gamma) z)$, where $j(p(\gamma), z)=c z$ $+d$ if $p(\gamma)=\left[\begin{array}{ll}a & b \\ c & d\end{array}\right] \in G L_{2}(\boldsymbol{R})$.

Let $\mathfrak{o}$ be a maximal order of $B$, and $\Gamma$ a congruence subgroup of $\mathfrak{o}_{+}^{\times}=\mathfrak{D} \cap$ $B_{+}^{\times}$. Following Shimura [18] 8.2, we define the space $\widetilde{S}_{n}(\Gamma, \Psi)$, the space of automorphic forms of weight $n$ with the representation $\Psi$ with respect to $\Gamma$, as the space of $C^{d}$-valued functions $f(z)$ on $H$ satisfying the following conditions:

(1.3.2) $f(z)$ is holomorphic,

(1.3.3) $f \mid[\gamma]_{n, \Psi}=f$ for all $\gamma \in \Gamma$.

Now let $\rho, m_{k}$ and $\Psi_{k}$ be as in 1.1 and 1.2. For $\Gamma$ as above, we make the following

Definition (1.3.4). We define the space $\subseteq(\Gamma, \rho)$ as the direct sum of the spaces $\widetilde{S}_{m_{k+2}}\left(\Gamma, \Psi_{k}\right)$ for $1 \leqslant k \leqslant r$, namely, $\mathfrak{S}(\Gamma, \rho)=\oplus_{k=1}^{r} \widetilde{S}_{m_{k+2}}\left(\Gamma, \Psi_{k}\right)$.

REMARK (1.3.5). Put $\Delta=\Gamma \cap F^{\times}$. Then for $c \in \Delta$, we easily see that $f \mid[c]_{m_{k+2}, \Psi_{k}}=\rho(c) f$ for any $k$ and $f \in \mathbb{S}_{m_{k+2}}\left(\Gamma, \Psi_{k}\right)$. Therefore, if $\rho(\Delta) \supseteq\{1\}$, we have $\mathfrak{S}(\Gamma, \rho)=\{0\}$.

REMARK (1.3.6). It is obvious that, up to the canonical isomorphism, the space $\mathfrak{S}(\Gamma, \rho)$ depends only on the equivalence class over $C$ of the representation: $G L_{2}^{+}(\boldsymbol{R}) \times\left(\boldsymbol{H}^{\times}\right)^{g-1} \rightarrow G L_{m}(\boldsymbol{R}) \smile G L_{m}(\boldsymbol{C})$. Let $j: \boldsymbol{H}^{\times} \longrightarrow G L_{2}(\boldsymbol{C})$ be the natural inclusion. Then it is easy to see that, as a representation over $C, \rho_{R}^{+}$is equivalent to a direct sum of the representations of the following form:

$$
\begin{gathered}
G L_{2}^{+}(\boldsymbol{R}) \times\left(H^{\times}\right)^{g-1} \ni\left(x_{1}, \cdots, x_{g}\right) \longmapsto \rho_{n_{1}}\left(x_{1}\right) \otimes \rho_{n_{2}}\left(j\left(x_{2}\right)\right) \\
\otimes \cdots \otimes \rho_{n_{g}}\left(j\left(x_{g}\right)\right) \operatorname{det}\left(x_{1}\right)^{\left(w-n_{1}\right) / 2} \prod_{i=2}^{g} \operatorname{det}\left(j\left(x_{i}\right)\right)^{\left(w-n_{i}\right) / 2}
\end{gathered}
$$

where $n_{i}(1 \leqslant i \leqslant g)$ are non-negative integers. The space of automorphic forms with respect to $\mathfrak{o}_{+}^{\times}$corresponding to this representation in the above 
sense is a subspace of $S\left(\mathfrak{o}^{\times} ; \chi_{0} ;\left\{n_{1}+2, n_{2}, \cdots, n_{g}\right\}\right)$ in the sense of Shimura [15] 3.2, with $\chi_{0}$ the trivial representation (or $\mathfrak{a}=\mathfrak{0}$ in the terminology of loc. cit.); we consider here the functions on $H$ instead of $\boldsymbol{C}-\boldsymbol{R}$.

1.4. We first recall and fix the adelic notation (cf. Shimura [17], [18]). We denote by $B_{A}^{\times}$the adelization of (the $Q$-algebraic group) $B^{\times}$, and by $B_{\infty}^{\times}$ (resp. $B_{f}^{\times}$) the infinite (resp. finite) component of $B_{A}^{\times} ; B_{A}^{\times}=B_{\infty}^{\times} \times B_{f}^{\times}$. Hence $B_{\infty}^{\times}=\left(B \otimes_{Q} R\right)^{\times}=G L_{2}(R) \times\left(H^{\times}\right)^{g-1}$. Let $B_{\infty+}^{\times}$be the connected component of the identity in $B_{\infty}^{\times}$, and put $B_{A+}^{\times}=B_{\infty+}^{\times} \times B_{f}^{\times}$. We denote by $\mathscr{Z}$ the family of the subgroups $S$ of $B_{A+}^{\times}$satisfying the following condition:

$$
S=B_{\infty+}^{\times} \times S_{0} \text { with an open compact subgroup } S_{0} \text { of } B_{f}^{\times} .
$$

Note that the above condition on $S_{0}$ is equivalent to saying that $S_{0}$ is an open subgroup of $\prod \mathfrak{o}_{p}^{\times}$, the product being taken over all the finite primes of $F$, with an order $\mathfrak{D}$ of $B$ (cf. [17] 2.22). For a member $S$ of $\mathscr{Z}$, we have a finite disjoint double coset decomposition (cf. [17] 3.5):

$$
B_{A+}^{\times}=\bigcup_{i=1}^{h} S x_{i} B_{+}^{\times}
$$

Fix $S \in \mathscr{Z}$ and the representatives $\left\{x_{i}\right\}$ as above, and put $S_{i}=x_{i}^{-1} S x_{i}$ and $\Gamma_{S_{i}}=S_{i} \cap B_{+}^{\times}$.

Definition (1.4.3). For $S \in \mathscr{Z}$ and $\left\{x_{i}\right\}$ as above, we define the space $\subseteq\left(S, \rho ;\left\{x_{i}\right\}\right)$ as the direct sum of $\subseteq\left(\Gamma_{S_{i}}, \rho\right)(1 \leqslant i \leqslant h): \subseteq\left(S, \rho ;\left\{x_{i}\right\}\right)=\bigoplus_{i=1}^{h} \subseteq\left(\Gamma_{S_{i}}, \rho\right)$.

REMARK (1.4.4). Let $B_{A+}^{\times}=\bigcup_{i=1}^{h} S x_{i} B_{+}^{\times}=\bigcup_{i=1}^{h} S y_{i} B_{+}^{\times}$be two decompositions of $B_{A+}^{\times}$, and assume that $S x_{i} B_{+}^{\times}=S y_{i} B_{+}^{\times}$for $1 \leqslant i \leqslant h$. Then we have $y_{i}=s_{i} x_{i} \alpha_{i}$ with $s_{i} \in S$ and $\alpha_{i} \in B_{+}^{\times}$. Put $S_{i}=x_{i}^{-1} S x_{i}$ and $S_{i}^{\prime}=y_{i}^{-1} S y_{i}$. Then it is easy to see that $f \mapsto f \mid\left[\alpha_{i}\right]_{m_{k+2}, \psi_{k}}$ determines an isomorphism of $\widetilde{S}_{m_{k}+2}\left(\Gamma_{S_{i}}, \Psi_{k}\right)$ onto $\widetilde{S}_{m_{k}+2}\left(\Gamma_{S_{i}}, \Psi_{k}\right)$, and that this isomorphism depends only on $x_{i}$ and $y_{i}$ (and not on the choice of $\left.\alpha_{i}\right)$. Hence $\subseteq\left(S, \rho ;\left\{x_{i}\right\}\right)$ and $\subseteq\left(S, \rho ;\left\{y_{i}\right\}\right)$ are naturally isomorphic. In the following, when we treat the space $\subseteq\left(S, \rho ;\left\{x_{i}\right\}\right)$ the representatives $\left\{x_{i}\right\}$ are assumed to be fixed, and we simply write it $\widetilde{\varsigma}(S, \rho)$.

REMARK (1.4.5). Since $\Gamma_{S_{i}} \cap F^{\times}=S \cap F^{\times}$for all $i$, we have, by (1.3.5), that $\widetilde{S}(S, \rho)=\{0\}$ if $\rho\left(S \cap F^{\times}\right) \supsetneq\{1\}$. Since this case is of no interest to us, we always assume that $\rho\left(S \cap F^{\times}\right)=\{1\}$ in the rest of this paper.

1.5. Hecke operators. Let $S=B_{\infty+}^{\times} \times S_{0} \in \mathscr{Z}$ be as in (1.4.1), and let o be a maximal order of $B$ such that $S_{0} \subseteq \prod \mathfrak{o}_{p}^{\times}$with the notation of loc. cit.. Let $\left\{\mathfrak{p}_{1}, \cdots, \mathfrak{p}_{n}\right\}$ be the (finite) set of primes of $F$ such that $S \nsupseteq \mathfrak{p}_{p_{i}}^{\times}$. Then we have $S=B_{\infty+}^{\times} \times \prod_{p \neq p_{i}} \mathfrak{a}_{p}^{\times} \times S_{0}^{\prime}$, where the middle product ranges over all the 
finite primes of $F$ excluding $\mathfrak{p}_{1}, \cdots, \mathfrak{p}_{n}$, and $S_{0}^{\prime}$ is an open subgroup of $\prod_{i=1}^{n} \mathfrak{b}_{\mathfrak{p} i}^{\times}$.

Definition (1.5.1). With the notation as above, we put

$$
\begin{aligned}
& L(S)=\prod_{i=1}^{n} \mathfrak{p}_{i}, \\
& V(S)=\left\{x \in B_{A+}^{\times} \mid x^{-1} S_{0}^{\prime} x=S_{0}^{\prime}\right\} .
\end{aligned}
$$

Note that $L(S)$ and $V(S)$ depend only on $S$ and not on the choice of $\mathfrak{o}$.

Recall the double coset decomposition $B_{A+}^{\times}=\bigcup_{i=1}^{h} S x_{i} B_{+}^{\times}$. We first prove the following

Proposition (1.5.2). For any $x \in V(S)$ and $i(1 \leqslant i \leqslant h)$, let $x x_{i}=s x_{j} \alpha$ with $s \in S$ and $\alpha \in B_{+}^{\times}$, and some $j(1 \leqslant j \leqslant h)$. Then the double coset $\Gamma_{S_{j}} \alpha \Gamma_{S_{i}}$ depends only on $S x S$.

Proof. For a fixed $i, j$ as above is uniquely determined by [17] 3.5. If $x x_{i}=s x_{j} \alpha=s^{\prime} x_{j} \alpha^{\prime}$ with $s, s^{\prime} \in S$ and $\alpha, \alpha^{\prime} \in B_{+}^{\times}$, one checks immediately that $\Gamma_{S_{j}} \alpha \Gamma_{S_{i}}=\Gamma_{S_{j}} \alpha^{\prime} \Gamma_{S_{i}}$. Let $x^{\prime}=s_{1} x s_{2}$ be another element of $S x S$, and let $x^{\prime} x_{i}=$ $s^{\prime} x_{j} \alpha^{\prime}$ with $s^{\prime} \in S$ and $\alpha^{\prime} \in B_{+}^{\times}$. Then it is easy to see that $\alpha^{\prime} \in S_{j} \alpha S_{i}$. If we can prove that $S_{j} \alpha S_{i}=S_{j} \alpha \Gamma_{S_{i}}$, then we have $\alpha^{\prime}=s_{j} \alpha \gamma$ with $s_{j} \in S_{j}$ and $\gamma \in \Gamma_{S_{i}}$, and hence $s_{j} \in \Gamma_{S_{j}}$, which will complete the proof. To prove the above assertion, we use [17] 2.19. Let $U=S_{i} \cap \alpha^{-1} S_{j} \alpha=x_{i}^{-1}\left(S \cap x^{-1} S x\right) x_{i}$. By loc. cit., it is sufficient to show that $\nu(U)=\nu(S)$, where $\nu$ denotes the reduced norm from $B_{A}^{\times}$to $F_{A}^{\times}$. But if $x$ is an element of $V(S)$, we have $S \cap x^{-1} S x=$ $B_{\infty+}^{\times} \times \prod_{p \neq p_{i}}\left(\mathfrak{o}_{\mathfrak{p}}^{\times} \cap x^{-1} \mathfrak{o}_{\mathfrak{p}}^{\times} x\right) \times S_{0}^{\prime}$.

Q.E.D.

REMARK (1.5.3). Let $x \in V(S)$ and let $i$ be an index such that $1 \leqslant i \leqslant h$. Then if $x x_{i}=s x_{j} \alpha$ as above, we easily have $\Gamma_{S_{j}} \alpha \Gamma_{S_{i}}=x_{j}^{-1} S x S x_{i} \cap B_{+}^{\times}$.

Let $x \in V(S)$. We now define a $C$-linear endomorphism $\mathfrak{I}(S x S)$ of $\widetilde{S}(S, \rho)$, the Hecke operator attached to $S x S$. Let $f=\left(f_{1}, \cdots, f_{h}\right)$ be an element of $\subseteq(S, \rho)=\bigoplus_{i=1}^{h} \subseteq\left(\Gamma_{S_{i}}, \rho\right)$, and let $f_{i}=\left(f_{i 1}, \cdots, f_{i r}\right)$ with $f_{i k} \in \widetilde{S}_{m_{k+2}}$ $\left(\Gamma_{S_{i}}, \Psi_{k}\right)$ (cf. (1.3.4)). We use the similar notation $g_{i}$ and $g_{i k}$ for other elements $g$ of $\mathfrak{S}(S, \rho)$.

DEFINITION (1.5.4). With the notation as above, $\mathfrak{T}(S x S) f=g$ is defined as follows. For any $i$, let $j$ and $\Gamma_{S_{j}} \alpha \Gamma_{S_{i}}$ be as in (1.5.2), and $\Gamma_{S_{j}} \alpha \Gamma_{S_{i}}=\cup_{l} \alpha_{l} \Gamma_{S_{i}}$ be a disjoint decomposition. Then we define $g_{j k}$ by $g_{j k}=\sum_{l} f_{i k} \mid\left[\alpha_{l}^{-1}\right]_{m_{k}+2, \Psi_{k}}$ (cf. (1.3.1)).

REMARK (1.5.5). If we change the representatives of the decomposition (1.4.2) as in (1.4.4), we have Hecke operators on $\subseteq\left(S, \rho ;\left\{x_{i}\right\}\right)$ and $\widetilde{S}\left(S, \rho ;\left\{y_{i}\right\}\right)$. It is easy to see that these operators are compatible with the natural isomorphism of $\subseteq\left(S, \rho ;\left\{x_{i}\right\}\right)$ onto $\subseteq\left(S, \rho ;\left\{y_{i}\right\}\right)$ defined in (1.4.4). 
REMARK (1.5.6). It follows easily from (1.5.3) that the decomposition $\Gamma_{S_{j}} \alpha \Gamma_{S_{i}}=\bigcup_{l} \alpha_{l} \Gamma_{S_{i}}$ (disjoint) is equivalent to the decomposition $S x S=$ $\cup_{l}\left(x_{j} \alpha_{l} x_{i}^{-1}\right) S$ (disjoint).

Let $\mathfrak{p}$ be a finite prime of $F$ which is prime to $L(S)$, i.e. $S \supseteq \mathfrak{o}_{p}^{\times}$using the notation as above. Take an element $x_{\mathfrak{p}}$ of $\mathfrak{o}_{\mathfrak{p}}$ whose reduced norm is a prime element of $F_{\mathfrak{p}}$, and let $x$ be an element of $B_{A+}^{\times}$whose $\mathfrak{p}$-component is $x_{\mathfrak{p}}$ and whose other components are all 1 . We then denote by $\mathfrak{I}_{S}(\mathfrak{p})=\mathfrak{I}(\mathfrak{p})$ the operator $\mathfrak{I}(S x S)$, which of course depends only on $\mathfrak{p}$. If moreover $\mathfrak{p}$ is prime to the discriminant $D(B / F)$ of $B$ over $F$, we denote by $\mathfrak{I}_{S}(\mathfrak{p}, \mathfrak{p})=\mathfrak{I}(\mathfrak{p}, \mathfrak{p})$ the operator $\mathfrak{I}(S \nu(x) S)$.

Definition (1.5.7). For a finite prime $\mathfrak{p}$ of $F$ which is prime to $L(S) D(B / F)$, we define the $p$-th Hecke polynominal $H_{\mathfrak{p}}(X ; \widetilde{S}(S, \rho))$ on $\widetilde{S}(S, \rho)$ by

$$
H_{\mathfrak{p}}(X ; \mathfrak{S}(S, \rho))=\operatorname{det}\left(1-\mathfrak{I}(\mathfrak{p}) X+N_{F / Q}(\mathfrak{p}) \mathfrak{I}(\mathfrak{p}, \mathfrak{p}) X^{2} \mid \widetilde{S}(S, \rho)\right),
$$

where $N_{F / Q}$ denotes the absolute norm.

By (1.5.5), $H_{p}(X ; \Im(S, \rho))$ depends only on $\mathfrak{p}, S$ and $\rho$.

REMARK (1.5.8). As we remarked in (1.3.6), when $S=B_{\infty+}^{\times} \times \prod \mathfrak{o}_{p}^{\times}$with a maximal order $\mathfrak{o}$ of $B, \mathcal{S}(S, \rho)$ is a direct sum of the subspaces of $S\left(\mathfrak{0}^{\times} ; \chi_{0}\right.$; $\left\{n_{i}\right\}$ )'s of [15] 3.2. The operators $\mathfrak{I}(\mathfrak{p})$ and $\mathfrak{I}(\mathfrak{p}, \mathfrak{p})$ above coincide with (the restrictions of) those given in loc. cit. if $w=0$. On the other hand, if we replace $\rho$ by $\rho^{\prime}=\rho \otimes N_{F / Q}\left(\nu_{B / F}()\right)^{a}$ with an integer $a, \rho^{\prime}$ also satisfies (1.1.1) and (1.1.2) with $w+2 a$ instead of $w$. For general $S$, the spaces $\subseteq(S, \rho)$ and $\Im\left(S, \rho^{\prime}\right)$ are the same, and a simple calculation shows that $H_{p}\left(X ; \widetilde{S}\left(S, \rho^{\prime}\right)\right)=$ $H_{\mathrm{p}}\left(N_{F / Q}(\mathfrak{p})^{a} X ; \Im(S, \rho)\right)$ for all $\mathfrak{p} \times D(B / F) L(S)$.

1.6. Let $S$ and $T$ be members of $\mathscr{Z}$, and assume that $T$ is contained in $S$. We consider here the relation between $\widetilde{S}(S, \rho)$ and $\mathfrak{S}(T, \rho)$, and the Hecke operators acting on them. The result of this subsection will be used in $\S 4$.

If $B_{A+}^{\times}=\bigcup_{i=1}^{h} S x_{i} B_{+}^{\times}$is a disjoint decomposition, we can choose $s_{1}, \cdots, s_{d}$ $\in S$ so that $B_{A+}^{\times}=\bigcup_{k=1}^{d} \bigcup_{i=1}^{h} T s_{k} x_{i} B_{+}^{\times}$(disjoint). Then we have $\left(s_{k} x_{i}\right)^{-1} T\left(s_{k} x_{i}\right)$ $\subseteq x_{i}^{-1} S x_{i}$ and hence $\subseteq\left(\Gamma_{S_{i}}, \rho\right)$ is contained in $\subseteq\left(B_{+}^{\times} \cap\left(s_{k} x_{i}\right)^{-1} T\left(s_{k} x_{i}\right), \rho\right)$. By the diagonal injection, we consider $\mathfrak{S}\left(\Gamma_{S_{i}}, \rho\right)$ as a subspace of $\oplus_{k=1}^{d} \mathfrak{S}\left(B_{+}^{\times} \cap\right.$ $\left.\left(s_{k} x_{i}\right)^{-1} T\left(s_{k} x_{i}\right), \rho\right)$, and hence $\subseteq(S, \rho)$ as a subspace of $\subseteq(T, \rho)$.

Proposition (1.6.1). Let $x$ be an element of $V(S) \cap V(T)$. For a maximal order $\mathfrak{v}$ as in 1.5, we assume that the $\mathfrak{p}$-component $x_{p}$ of $x$ is contained in $\mathfrak{D}_{\mathfrak{p}}^{\times}$for all $\mathfrak{p} \mid L(T) L(S)^{-1}$. Then the restriction of $\mathfrak{I}(T x T)$ to $\mathfrak{S}(S, \rho)$ coincides with $\mathfrak{I}(S x S)$. 
Proof. Put $y_{I}=s_{k} x_{i}$ and $\Gamma_{T_{I}}=y_{I}^{-1} T y_{I} \cap B_{+}^{\times}$. For an element $x \in V(S) \cap$ $V(T)$ as above, let $x y_{I}=t y_{J} \alpha$ with $t \in T, \alpha \in B_{+}^{\times}$, and $y_{J}=s_{m} x_{j}$ with some $m$ and $j$, and let $\Gamma_{T_{J}}$ be defined similarly as above for $y_{J}$. To prove our assertion, it is sufficient to show that $\Gamma_{T_{J}} \alpha \Gamma_{T_{I}}=\bigcup_{l=1}^{e} \alpha_{l} \Gamma_{T_{I}}$ (disjoint) implies $\Gamma_{S_{j}} \alpha \Gamma_{s_{i}}=\bigcup_{l=1}^{e} \alpha_{l} \Gamma_{S_{i}}$ (disjoint).

We first prove that $T \cap\left(S \cap x S x^{-1}\right)=T \cap x T x^{-1}$. Write $T=B_{\infty}^{\times} \times \prod_{p \nmid L(T)}$ $\mathfrak{o}_{\mathfrak{p}}^{\times} \times T_{0}^{\prime}$ as in 1.5 , where the middle product ranges over all the finite primes of $F$ which are prime to $L(T)$. Take an element $t=\left(t_{\infty}, t_{1}, t^{\prime}\right) \in T \cap\left(S \cap x S x^{-1}\right)$ with $t_{\infty} \in B_{\infty+}^{\times}, t_{1} \in \prod_{\mathfrak{p} \backslash L(T)} \mathfrak{o}_{\mathfrak{p}}^{\times}$, and $t^{\prime} \in T_{0}^{\prime}$. If $x=\left(x_{\infty}, x_{1}, x^{\prime}\right) \in V(T)$ with the similar notation, we have $x_{\infty}^{-1} t_{\infty} x_{\infty} \in B_{\infty+}^{\times}, x_{1}^{-1} t_{1} x_{1} \in \prod_{\mathfrak{p} \backslash L(T)} \mathfrak{D}_{p}^{\times}$, and $x^{\prime-1} t^{\prime} x^{\prime} \in T_{0}^{\prime}$ by the assumption on $t$. Hence $t \in x T x^{-1}$ and $t \in T \cap x T x^{-1}$. Since the other inclusion is obvious, we have proved our assertion. Next, write $S=B_{\infty+}^{\times} \times$ $\prod_{p \nmid L(T)} \mathfrak{o}_{p}^{\times} \times S_{0}^{\prime \prime}$ with $S_{0}^{\prime \prime} \subseteq \prod_{\mathfrak{p} \mid L(T)} \mathfrak{b}_{\mathfrak{p}}^{\times}$. Then we have $\left[S \cap x S x^{-1}: T \cap x T x^{-1}\right]=$ $\left[S_{0}^{\prime \prime} \cap x S_{0}^{\prime \prime} x^{-1}: T_{0}^{\prime} \cap x T_{0}^{\prime} x^{-1}\right]$. But since $S_{0}^{\prime \prime}$ is a product of $S_{0}^{\prime}$ and $\mathfrak{b}_{p}^{\times}$with $\mathfrak{p} \mid L(T) L(S)^{-1}$, we have $S_{0}^{\prime \prime} \cap x S_{0}^{\prime \prime} x^{-1}=S_{0}^{\prime \prime}$ by our assumption on $x$. Since $T_{0}^{\prime} \cap x T_{0}^{\prime} x^{-1}=T_{0}^{\prime}$, we have proved that $\left[S \cap x S x^{-1}: T \cap x T x^{-1}\right]=[S: T]$, and hence $\left[S: S \cap x S x^{-1}\right]=\left[T: T \cap x T x^{-1}\right]$.

By (1.5.6), we have $T x T=\bigcup_{l=1}^{e} \xi_{l} T$ (disjoint) with $\xi_{l}=y_{J} \alpha_{l} y_{I}^{-1}$. This is equivalent to saying that $T=\bigcup_{l=1}^{e}\left(\xi_{l} x^{-1}\right)\left(T \cap x T x^{-1}\right)$ (disjoint). By what we have said above, we have $S=\bigcup_{l=1}^{e}\left(\xi_{l} x^{-1}\right)\left(S \cap x S x^{-1}\right)$ (disjoint), and hence $S x S=\bigcup_{l=1}^{e} \xi_{l} S=\bigcup_{l=1}^{e} s_{m} x_{j} \alpha_{l} x_{i}^{-1} S$ (disjoint). This together with (1.5.6) completes the proof.

Q.E.D.

CoRollary (1.6.2). Let $\mathfrak{p}$ be a finite prime of $F$ which is prime to $L(T)$. Then the restriction of $\mathfrak{I}_{T}(\mathfrak{p})$ to $\mathfrak{S}(S, \rho)$ coincides with $\mathfrak{I}_{S}(\mathfrak{p})$. If moreover $\mathfrak{p}$ is prime to $D(B / F)$, the restriction of $\mathfrak{I}_{T}(\mathfrak{p}, \mathfrak{p})$ to $\mathfrak{S}(S, \rho)$ coincides with $\mathfrak{I}_{S}(\mathfrak{p}, \mathfrak{p})$.

Next, we assume that $T$ is a normal subgroup of $S$. In this case, $S$ is contained in $V(T)$, and hence $\mathfrak{I}(T s T)$ are defined for all $s \in S$. It is easy to see that $s \mapsto \mathfrak{I}(T s T)$ is a homomorphism of $S /\left(S \cap F^{\times}\right) T$ into $\operatorname{End}(\subseteq(T, \rho))$.

Corollary (1.6.3). If $T$ is a normal subgroup of $S$, we have $\subseteq(S, \rho)=$ $\widetilde{S}(T, \rho)^{\left(S /\left(S \cap F^{\times}\right) T\right)}$, the set of the elements of $\widetilde{S}(T, \rho)$ which are invariant under all $\mathfrak{I}(T s T)$ with $s \in S$.

Proof. Any $s \in S$ satisfies the condition of (1.6.1), and hence $\subseteq(S, \rho)$ is invariant under $\mathfrak{I}(T s T)$ by (1.6.1). On the other hand, it is a direct computation to show that $\widetilde{S}(T, \rho)^{\left(S /\left(S \cap F^{\times}\right) T\right)}$ is contained in $\widetilde{S}(S, \rho)$, and the details may be left to the reader.

Q.E.D.

1.7. Petersson inner product. In this subsection, we study the Petersson inner product on our space $\subseteq(S, \rho)$ following Shimura [15]. We keep the notation of the previous subsections. 
Let $\sigma_{k}(1 \leqslant k \leqslant r)$ be as in (1.2.2), and let $d_{k}$ be its dimension; $\sigma_{k}:\left(\boldsymbol{H}^{u}\right)^{g-1}$ $\rightarrow G L_{d_{k}}(\boldsymbol{R})$. Since $\left(\boldsymbol{H}^{u}\right)^{g-1}$ is compact, there is a positive definite symmetric matrix $\boldsymbol{\Xi}_{k} \in G L_{d_{k}}(R)$ which satisfies

$$
{ }^{t} \sigma_{k}(h) \Xi_{k} \sigma_{k}(h)=\Xi_{k} \text { for all } h \in\left(H^{u}\right)^{g-1} .
$$

We fix such a matrix $\Xi_{k}$ for each $k$.

Now let $f=\left(f_{i k}\right)$ and $g=\left(g_{i k}\right)$ be elements of $\mathfrak{S}(S, \rho)=\oplus_{i=1}^{h} \oplus_{k=1}^{r} \widetilde{S}_{m_{k+2}}\left(\Gamma_{S_{i}}\right.$, $\Psi_{k}$ ) with $f_{i k}, g_{i k} \in \widetilde{S}_{m_{k}+2}\left(\Gamma_{S_{i}}, \Psi_{k}\right)$.

Definition (1.7.2). For $f$ and $g$ as above, we define the Petersson inner product $\langle f, g\rangle_{s}$ of $f$ and $g$ by

$$
\begin{aligned}
\langle f, g\rangle_{S} & =\sum_{i=1}^{n} \sum_{k=1}^{r}\left\langle f_{i k}, g_{i k}\right\rangle_{\Gamma_{S_{i}}, k}, \\
\left\langle f_{i k}, g_{i k}\right\rangle_{\Gamma_{S_{i}}, k} & =\int_{H / \Gamma_{S_{i}}}{ }^{t} f_{i k}(z) \Xi_{k} \overline{g_{i k}(z)} y^{m_{k}} d x d y,
\end{aligned}
$$

where $z=x+y \sqrt{-1}$, and the integral is over a fundamental domain of $H / \Gamma_{S_{i}}$.

It is easy to see that this is in fact well-defined, i.e. the integral does not depend on the choice of a fundamental domain. Of course, $\langle,\rangle_{S}$ gives a non-degenerate hermitian form on $\mathfrak{S}(S, \rho)$, but in the following, we consider it as a pairing between $\subseteq(S, \rho)$ and another space. To state this, let $\iota$ denote the canonical involution of $B$ or $B_{A+}^{\times}$. Then $B_{A+}^{\times}=\bigcup_{i=1}^{h} S x_{i} B_{+}^{\times}$implies $B_{A+}^{\times}=\bigcup_{i=1}^{h} S^{c} x_{i}^{-\imath} B_{+}^{\times}$, where $x_{i}^{-\imath}=\left(x_{i}^{-1}\right)^{\iota}=\left(x_{i}^{c}\right)^{-1}$. Then it is obvious that $\left(x_{i}^{-c}\right)^{-1} S^{\imath} x_{i}^{-\imath} \cap B_{+}^{\times}=\left(\Gamma_{S_{i}}\right)^{c}$ and that $\subseteq\left(\Gamma_{S_{i}}, \rho\right)=\subseteq\left(\left(\Gamma_{S_{i}}\right)^{\imath}, \rho\right)$. Hence we have $\widetilde{S}\left(S, \rho ;\left\{x_{i}\right\}\right)=\subseteq\left(S^{\iota}, \rho ;\left\{x_{i}^{-\imath}\right\}\right)$.

Proposition (1.7.3). The Petersson inner product defines a pairing

$$
\langle,\rangle_{S}: \Im\left(S, \rho ;\left\{x_{i}\right\}\right) \times \Im\left(S^{\imath}, \rho ;\left\{x_{i}^{-\imath}\right\}\right) \longrightarrow C
$$

which satisfies $\langle\mathfrak{T}(S x S) f, g\rangle_{S}=\left\langle f, \mathfrak{I}\left(S^{\prime} x^{\imath} S^{t}\right) g\right\rangle_{S}$ for all $x \in V(S)$.

Proof. First note that $x \in V(S)$ implies $x^{i} \in V\left(S^{t}\right)$, and the both sides of the equality are meaningful. Let us fix an index $i$ and determine $j$ and $\alpha$ from $x$ as in (1.5.2). Let $\Gamma_{S_{j}} \alpha \Gamma_{S_{i}}=\bigcup_{l} \alpha_{l} \Gamma_{S_{i}}$ and $\Gamma_{S_{i}} \alpha^{-1} \Gamma_{S_{j}}=\bigcup_{m} \beta_{m} \Gamma_{S_{j}}$ be disjoint decompositions. Take elements $f_{i k} \in \mathbb{S}_{m_{k+2}}\left(\Gamma_{S_{i}}, \Psi_{k}\right)$ and $g_{j k} \in \mathbb{S}_{m_{k+2}}\left(\Gamma_{S_{j}}\right.$, $\Psi_{k}$ ). Then a computation similar to that of [15] 3.5 yields:

$$
\left\langle\sum_{l} f_{i k} \mid\left[\alpha_{l}^{-1}\right], g_{j k}\right\rangle_{\Gamma_{S_{j}, k}}=N_{F / Q}(\nu(\alpha))^{w}\left\langle f_{i k}, \sum_{m} g_{j k} \mid\left[\beta_{m}^{-1}\right]\right\rangle_{\Gamma_{S_{i}, k}} .
$$

But for any totally positive element $c \in F^{\times}$, we have $f_{i k} \mid[c]=N_{F / Q}(c)^{-w} f_{i k}$, and hence the above is equal to $\left\langle f_{i k}, \sum_{m} g_{j k} \mid\left[\left(\nu(\alpha) \beta_{m}\right)^{-1}\right]\right\rangle_{T_{i}, k}$, the summation in the blacket being taken over the representatives of the right coset decomposition 
of $\Gamma_{S_{i}} \nu(\alpha) \alpha^{-1} \Gamma_{S_{j}}=\Gamma_{S_{i}} \alpha^{t} \Gamma_{S_{j}}$. On the other hand, let $\Gamma_{S_{i}}^{t} \alpha^{t} \Gamma_{S_{j}}^{t}=\cup_{n} \gamma_{n} \Gamma_{S_{j}}$. Then it is easy to see that $\sum_{m} f\left|\left[\left(\nu(\alpha) \beta_{m}\right)^{-1}\right]_{m_{k}+2, w_{k}}=\sum_{n} f\right|\left[\gamma_{n}^{-1}\right]_{m_{k+2}, w_{k}}$ for all $f \in \widetilde{S}_{m_{k+2}}$ $\left(\Gamma_{S_{j}}, \Psi_{k}\right)=\widetilde{S}_{m_{k+2}}\left(\Gamma_{S_{j}}^{t}, \Psi_{k}\right)$. By (1.5.3), we have $\Gamma_{S_{j}} \alpha \Gamma_{S_{i}}=x_{j}^{-1} S x S x_{i} \cap B_{+}^{\times}$, and hence $\Gamma_{S_{i}}^{\iota} \alpha^{t} \Gamma_{S_{j}}^{\iota}=\left(x_{i}^{-\imath}\right)^{-1} S^{t} x^{t} S^{t} x_{j}^{-t} \cap B_{+}^{\times}$, which completes the proof. Q.E.D.

Next, we suppose that there is an element $\eta \in B_{+}^{\times}$such that $\eta^{-1} S \eta=S^{c}$. Then we have $\mathfrak{S}\left(S^{i}, \rho ;\left\{\eta^{-1} x_{i} \eta\right\}\right)=\oplus_{k=1}^{r} \oplus_{i=1}^{h} \mathbb{S}_{m_{k+2}}\left(\eta^{-1} \Gamma_{S_{i}} \eta, \Psi_{k}\right)$, and $\mathbb{S}_{m_{k+2}}\left(\Gamma_{S_{i}}\right.$, $\left.\Psi_{k}\right) \ni f \mapsto f \mid[\eta] \in \mathbb{S}_{m_{k+2}}\left(\eta^{-1} \Gamma_{S_{i}} \eta, \Psi_{k}\right)$ determines an isomorphism $I_{\eta}: \mathcal{S}\left(S, \rho ;\left\{x_{i}\right\}\right)$ $\widetilde{\rightarrow}\left(S^{\imath}, \rho ;\left\{\eta^{-1} x_{i} \eta\right\}\right)$. With this notation, we have the following easy

LEMMA (1.7.4). For any $x \in V(S)$, we have $\eta^{-1} x \eta \in V\left(S^{\prime}\right)$ and $I_{\eta} \circ \mathfrak{I}(S x S)$ $=\mathfrak{I}\left(S^{\imath}\left(\eta^{-1} x \eta\right) S^{\imath}\right) \circ I_{\eta}$.

Summing up we have the following

Corollary (1.7.5). Suppose that there is an element $\eta \in B_{+}^{\times}$such that $\eta^{-1} S \eta=S^{t}$. Then the Hecke polynomials $H_{p}(X ; \widetilde{S}(S, \rho))((1.5 .7))$ are polynomials with real coefficients.

\section{§. Cohomology groups attached to $\widetilde{S}(S, \rho)$}

As in $\S 1$, we fix a representation $\rho$ satisfying (1.1.1) and (1.1.2), and consider the spaces $\subseteq(S, \rho)$ for subgroups $S \in \mathscr{Z}$ of $B_{A+}^{\times}$. We keep the notation used in $\S 1$. In this section, we first study the Eichler-Shimura cohomology groups (cf. [15], [18]), and then the étale cohomology groups introduced by Langlands [10], attached to $\subseteq(S, \rho)$.

2.1. Eichler-Shimura cohomology groups. Let $f=\left(f_{i k}\right)$ be an element of $\widetilde{S}(S, \rho)$ with $f_{i k} \in \widetilde{S}_{m_{k+2}}\left(\Gamma_{S_{i}}, \Psi_{k}\right)(1 \leqslant i \leqslant h, 1 \leqslant k \leqslant r)$ using the notation as in $\S 1$. Denoting by $d_{k}$ the dimension of the representation $\Psi_{k}$, we let $\omega_{i k}$ be the $\boldsymbol{R}^{d_{k}} \otimes \boldsymbol{R}^{m_{k}+1}$-valued 1-form $\operatorname{Re}\left(f_{i k} \otimes{ }^{t}\left[z^{m_{k}}, \cdots, z, 1\right] d z\right)$ on $H$, where Re means the real part. Then the automorphy condition (1.3.3) implies

$$
\omega_{i k} \circ \alpha=\left(\operatorname{det}(p(\alpha))^{-m_{k} / 2} \Psi_{k}(\alpha) \otimes \rho_{m_{k}}(\alpha)\right) \omega_{i k} \quad \text { for all } \quad \alpha \in \Gamma_{S_{i}} .
$$

Identifying $\boldsymbol{R}^{m}$, the representation space of $\rho$, with $\oplus_{k=1}^{r} \boldsymbol{R}^{d_{k}} \otimes \boldsymbol{R}^{m_{k}+1}$, we see that $\left(\omega_{i 1}, \cdots, \omega_{i r}\right)$ is an $\boldsymbol{R}^{m}$-valued 1 -form on $H$ which satisfies

$$
\left(\omega_{i 1}, \cdots, \omega_{i r}\right) \circ \alpha=\rho(\alpha)\left(\omega_{i 1}, \cdots, \omega_{i r}\right) \text { for all } \alpha \in \Gamma_{s_{i}} .
$$

Put $\bar{\Gamma}_{S_{i}}=\Gamma_{S_{i}} / S_{i} \cap F^{\times}$. Since we have assumed that $\rho\left(\Gamma_{S_{i}} \cap F^{\times}\right)=\{1\}$ ((1.4.5)), $\boldsymbol{R}^{m}$ is a $\bar{\Gamma}_{S_{i}}$-module by $\rho$. We then have a natural $\boldsymbol{R}$-linear homomorphism

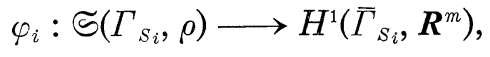


which sends $f_{i k} \in \widetilde{S}_{m_{k+2}}\left(\Gamma_{S_{i}}, \Psi_{k}\right)$ to the class of the 1-cocycle $\gamma \mapsto \int_{z_{0}}^{\gamma\left(z_{0}\right)} \omega_{i k}$ in $H^{1}\left(\bar{\Gamma}_{S_{i}}, \boldsymbol{R}^{d_{k}} \otimes \boldsymbol{R}^{m_{k}+1}\right)$ with the above notation (cf. [18] 8.2). Here of course, we denote by $H^{1}\left(\bar{\Gamma}_{S_{i}}, \boldsymbol{R}^{m}\right)$ the usual group cohomology.

Proposition (2.1.4). $\quad \varphi_{i}$ is an isomorphism of $\boldsymbol{R}$-vector spaces for each $i$ $(1 \leqslant i \leqslant h)$, and hence taking the direct sum, we have a natural isomorphism

$$
\varphi: \Im(S, \rho) \stackrel{\sim}{\longrightarrow} \bigoplus_{i=1}^{h} H^{1}\left(\bar{\Gamma}_{S_{i}}, \boldsymbol{R}^{m}\right)
$$

of $\boldsymbol{R}$-vector spaces.

Proof. By 1.2, each $\Psi_{k}$ maps $\Gamma_{s_{i}}$ into a compact subgroup of $G L_{d_{k}}(\boldsymbol{R})$, and hence our assertion is a special case of [18] Th. 8.4.

Q.E.D.

We next translate the action of the Hecke operators to the cohomology groups. We take an element $x \in V(S)((1.5 .1))$, and let $\mathfrak{I}(S x S)_{i j}: \mathfrak{S}\left(\Gamma_{S_{i}}, \rho\right) \rightarrow$ $\mathfrak{S}\left(\Gamma_{S_{j}}, \rho\right)$ be the restriction of $\mathfrak{I}(S x S)$ to $\mathfrak{S}\left(\Gamma_{S_{i}}, \rho\right)$ with the notation of (1.5.4).

Proposition (2.1.5). The notation being as above, let $x x_{i}=s x_{j} \alpha$ be as in (1.5.2). We define the map

$$
\mathfrak{I}^{\prime}(S x S)_{i j}: H^{1}\left(\bar{\Gamma}_{S_{i}}, \boldsymbol{R}^{m}\right) \longrightarrow H^{1}\left(\bar{\Gamma}_{S_{j}}, \boldsymbol{R}^{m}\right)
$$

as the composite of the following three maps:

(i) the restriction: $H^{1}\left(\bar{\Gamma}_{S_{i}}, \boldsymbol{R}^{m}\right) \longrightarrow H^{1}\left(\bar{\Gamma}_{S_{i}}, \cap \alpha^{-1} \Gamma_{S_{j}} \alpha, \boldsymbol{R}^{m}\right)$,

(ii) the corestriction: $H^{1}\left(\Gamma_{S_{i}} \cap \alpha^{-1} \Gamma_{S_{j}} \alpha, \boldsymbol{R}^{m}\right) \longrightarrow H^{1}\left(\alpha^{-1} \Gamma_{S}{ }^{1} \alpha, \boldsymbol{R}^{m}\right)$,

(iii) the map: $\left.H^{1} \overline{\left(\alpha^{-1} \Gamma_{S_{j}} \alpha\right.}, \boldsymbol{R}^{m}\right) \longrightarrow H^{1}\left(\bar{\Gamma}_{S_{j}}, \boldsymbol{R}^{m}\right)$ which corresponds to the homomorphism $\rho(\alpha): \boldsymbol{R}^{m} \rightarrow \boldsymbol{R}^{m}$ and the change of the group $\Gamma_{S_{j}} \rightarrow \alpha^{-1} \Gamma_{S_{j}} \alpha$ which sends $\gamma$ to $\alpha^{-1} \gamma \alpha$.

Then we have $\mathfrak{I}^{\prime}(S x S)_{i j} \circ \varphi_{i}=\varphi_{j} \circ \mathfrak{I}(S x S)_{i j}$ for all $i$.

Proof. This is nothing more than a reformulation of [18] Prop. 8.5. Indeed, first recall the decomposition $\Gamma_{S_{j}} \alpha \Gamma_{S_{i}}=\bigcup_{l=1}^{e} \alpha_{l} \Gamma_{S_{i}}$ (disjoint). For $\gamma \in \Gamma_{s_{j}}$ and $l$, define $\gamma_{l}^{\prime} \in \Gamma_{S_{i}}$ by the relation $\gamma^{-1} \alpha_{l}=\alpha_{l^{\prime}} \gamma_{l}^{\prime-1}$ with some $l^{\prime}$. For an $\boldsymbol{R}^{m}$-valued 1-cocycle $u$ on $\bar{\Gamma}_{S_{i}}$, we see by a direct computation that the image of the class of $u$ under $\mathfrak{I}^{\prime}(S x S)_{i j}$ is the class of the 1-cocycle which sends $\gamma$ to $\sum_{l=1}^{e} \rho\left(\alpha_{l}\right) u\left(\gamma_{l}^{\prime}\right)$. One then deduces our assertion as in [18] Prop. 8.5.

Q.E.D.

We define the endomorphism $\mathbb{I}^{\prime}(S x S)$ of $\oplus_{i=1}^{h} H^{1}\left(\bar{\Gamma}_{S_{i}}, \boldsymbol{R}^{m}\right)$ as the direct sum of $\mathfrak{I}^{\prime}(S x S)_{i j} ; \mathfrak{I}^{\prime}(S x S)=\bigoplus_{i=1}^{h} \mathfrak{I}^{\prime}(S x S)_{i j}$.

To construct $l$-adic representations, we need the existence of certain lattices in $\boldsymbol{R}^{m}$, and we consider the following condition on $\rho$ :

(2.1.6) For a maximal order o of $B$, there exists a Z-lattice $X$ in $\boldsymbol{R}^{m}$ which 
satisfies the following two conditions:

(2.1.6.1) For any $\alpha \in \mathfrak{D} \cap B^{\times}, X$ is $\rho(\alpha)$-stable.

(2.1.6.2) For any positive integer $a$ and any $\alpha \in \mathfrak{D} \cap B^{\times}$such that $\alpha-1 \in$ an, we have $(\rho(\alpha)-\rho(1)) X \subseteq a X$.

REMARK (2.1.7). We note that the validity of (2.1.6) for one $\mathfrak{D}$ implies the validity of it for other maximal orders. The existence of $X$ satisfying (2.1.6.1) is proved to be automatic. But we do not know whether (2.1.6.2) is automatic in general. Examples of such $\rho$ (together with an additional property (4.1.1)) will be discussed in $\S 5$.

Now suppose that $\rho$ satisfies (2.1.6) and fix a prime number $l$. Then, by (1.1.1), we see that $X \otimes_{Z} Z_{l}$ is an $\mathfrak{o}_{l}^{\times}=\left({ }^{\circ} \otimes_{Z} Z_{l}\right)^{\times}$-module, and $X \otimes_{Z}\left(R \times \prod_{p} Z_{p}\right)$ is an $\left(\mathfrak{o} \otimes_{Z}\left(R \times \prod_{p} Z_{p}\right)\right)^{\times}$-module by means of $\rho$. Hence if $S=B_{\infty+}^{\times} \times S_{0} \in \mathscr{Z}$ with $S_{0} \subseteq \prod \mathfrak{p}_{\mathfrak{p}}^{\times}$, then the latter module appearing in the above is an $S$-module by $\rho$. Moreover if we denote by $A_{l}$ the elements of $B_{A+}^{\times}$whose -components are contained in $\mathfrak{b}_{\mathfrak{l}}^{\times}$for all primes $\mathfrak{l}$ of $F$ above $l, X \otimes_{Z} Z_{l}$ is an $A_{l}$-module by $\rho$. In the following, we always assume that, in the double coset decomposition (1.4.2), $x_{i}$ are taken from $A_{l}$ (which is of course possible). In this case, $X / l^{n} X$ is a $\bar{\Gamma}_{S_{i}}$-module for each $i(1 \leqslant i \leqslant h)$, and we can define the operator $\mathfrak{I}^{\prime}(S x S)=\oplus_{i=1}^{h} \mathfrak{I}^{\prime}(S x S)_{i j}$ on $\oplus_{i=1}^{h} H^{1}\left(\bar{\Gamma}_{S_{i}}, X / l^{n} X\right)$ exactly in the same manner as in (2.1.5) for any $x \in A_{l} \cap V(S)$.

2.2. Let $\Gamma_{1}$ and $\Gamma_{2}$ be subgroups of $P G L_{2}(R)$ which give Fuchsian groups of the first kind acting on $H$. We suppose that $\Gamma_{1}$ and $\Gamma_{2}$ do not have elliptic elements. Let $\alpha$ be an element of $P G L_{2}(R)$, and put $\Gamma_{3}=\Gamma_{1} \cap \alpha^{-1} \Gamma_{2} \alpha$. We suppose that the indices of $\Gamma_{3}$ in $\Gamma_{1}$ and $\alpha^{-1} \Gamma_{2} \alpha$ are finite.

For any (discrete) $\Gamma_{1}$-module $M$, the natural quotient $H \times M / \Gamma_{1}$ is (the étale space attached to) a sheaf on $H / \Gamma_{1}$. We denote by $H^{i}\left(H / \Gamma_{1}, H \times M / \Gamma_{1}\right)$ the usual cohomology groups of $H / \Gamma_{1}$ with coefficients in this sheaf, and use similar notation for $\Gamma_{2}$ and $\Gamma_{3}$. For any $\Gamma_{1}$-module $M$, we have a cartesian diagram:

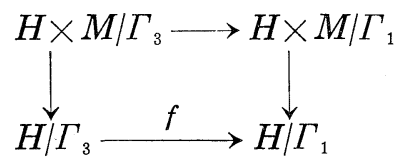

where all the maps are natural projections. This diagram gives a canonical isomorphism of $H \times M / \Gamma_{3}$ onto $f^{*}\left(H \times M / \Gamma_{1}\right)$, the inverse image by $f$, and hence we have the canonical map:

$$
c^{i}: H^{i}\left(H / \Gamma_{1}, H \times M / \Gamma_{1}\right) \longrightarrow H^{i}\left(H / \Gamma_{3}, H \times M / \Gamma_{3}\right) .
$$

Next, for any $\alpha^{-1} \Gamma_{2} \alpha$-module $M$, we have a similar cartesian diagram: 


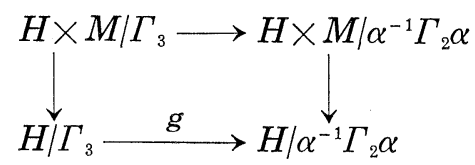

from which we obtain a canonical isomorphism of $H \times M / \Gamma_{3}$ onto $g^{*}(H \times$ $M / \alpha^{-1} \Gamma_{2} \alpha$ ). We then have the trace map for the cohomology (cf. [1] IX 5, XVII 6.2):

$$
t^{i}: H^{i}\left(H / \Gamma_{3}, H \times M / \Gamma_{3}\right) \longrightarrow H^{i}\left(H / \alpha^{-1} \Gamma_{2} \alpha, H \times M / \alpha^{-1} \Gamma_{2} \alpha\right) .
$$

Finally, suppose that $M$ is a $\left\langle\Gamma_{2}, \alpha\right\rangle$-module and consider the cartesian dialgram:

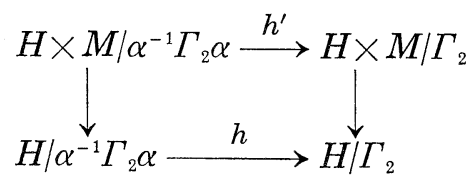

where $h$ (resp. $h^{\prime}$ ) sends the class of $z \in H$ (resp. $(z, m) \in H \times M$ ) to the class of $\alpha z$ (resp. $(\alpha z, \alpha m)$ ). From this diagram, we obtain a canonical isomorphism of $H \times M / \alpha^{-1} \Gamma_{2} \alpha$ onto $h^{*}\left(H \times M / \Gamma_{2}\right)$, and hence we have the trace map:

$$
t^{\prime i}: H^{i}\left(H / \alpha^{-1} \Gamma_{2} \alpha, H \times M / \alpha^{-1} \Gamma_{2} \alpha\right) \longrightarrow H^{i}\left(H / \Gamma_{2}, H \times M / \Gamma_{2}\right) .
$$

Lemma (2.2.4). Let $\Gamma$ be one of $\Gamma_{k}(1 \leqslant k \leqslant 3)$. Then for any $\Gamma$-module $M$, there is a natural isomorphism: $H^{i}(\Gamma, M) \stackrel{\sim}{\longrightarrow} H^{i}(H / \Gamma, H \times M / \Gamma)$ for $i=0$ or 1 , and the following diagram commutes for any $\left\langle\Gamma_{1}, \Gamma_{2}, \alpha\right\rangle$-module $M$ and for $i=0$ or 1 :

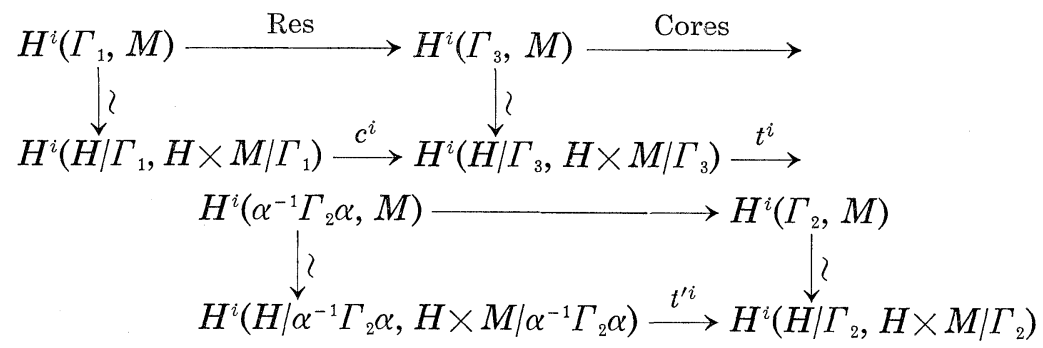

where the upper horizontal arrows are defined as in (2.1.5), and the lower ones are defined by (2.2.1)-(2.2.3).

Proof. The first assertion is well known; the assertion for $H^{0}$ is obvious, and both $H^{1}$ classify principal homogeneous spaces under $H \times M / \Gamma$ over $H / \Gamma$, since $H$ is simply connected. This defines an isomorphism of exact 
o-functors $M \mapsto\left\{H^{i}(\Gamma, M)\right\}_{i=0,1}$ and $M \mapsto\left\{H^{i}(H \mid \Gamma, H \times M / \Gamma)\right\}_{i=0,1}$ from the category of $\Gamma$-modules.

We now prove the commutativity of the middle square; the rest can be proved similarly. It is known that $M \mapsto\left\{H^{i}\left(\Gamma_{3}, M\right)\right\}$ is a universal $\partial$-functor from the category of $\alpha^{-1} \Gamma_{2} \alpha$-modules to that of abelian groups. On the other hand, the corestriction and the trace map are morphisms of $\partial$-functors. Hence to prove the commutativity, it is sufficient to show it for $H^{0}$. This can be checked easily from the definitions of the corestriction and the trace map.

Q.E.D.

Now let us return to the situation considered in 2.1. There, we have defined the operator $\mathfrak{I}^{\prime}(S x S)_{i j}$ for the group cohomologies with coefficients in $\boldsymbol{R}^{m}$ or $X / l^{n} X$ under certain conditions.

Corollary (2.2.5). Suppose that $\rho$ satisfies (2.1.6), and let $M$ be either $\boldsymbol{R}^{m}$ or $X \mid l^{n} X$. Take an element $x \in V(S)$ which is assumed to lie in $A_{l}$ when $M=X / l^{n} X$. Fix $i$, and let $j$ and $\alpha$ be as in (1.5.2) for $x$. Suppose also that $\Gamma_{S_{i}}$ and $\Gamma_{S_{j}}$ have no non-trivial elliptic elements. We then define the operator

$$
\mathfrak{I}^{\prime \prime}(S x S)_{i j}: H^{1}\left(H / \Gamma_{S_{i}}, H \times M / \Gamma_{S_{i}}\right) \longrightarrow H^{1}\left(H / \Gamma_{S_{j}}, H \times M / \Gamma_{S_{j}}\right)
$$

as the composite of the following two maps:

(i) The canonical map: $H^{1}\left(H / \Gamma_{S_{i}}, H \times M / \Gamma_{S_{i}}\right) \rightarrow H^{1}\left(H / \Gamma_{S_{i}} \cap \alpha^{-1} \Gamma_{S_{j}} \alpha\right.$, $\left.H \times M / \Gamma_{S_{i}} \cap \alpha^{-1} \Gamma_{S_{j}} \alpha\right)$ defined in the same way as (2.2.1).

(ii) Consider the cartesian diagram:

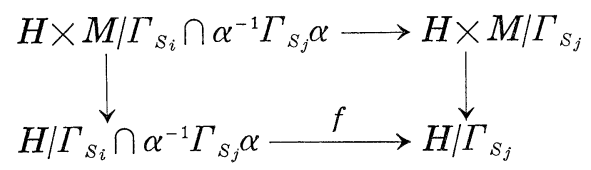

where two vertical maps are natural projections, and the upper (resp. the lower) horizontal map sends the class of $(z, m) \in H \times M$ (resp. $z \in H$ ) to the class of $(p(\alpha) z, \rho(\alpha) m)$ (resp. $p(\alpha) z)$. This diagram gives the canonical isomorphism of $H \times M / \Gamma_{S_{i}} \cap \alpha^{-1} \Gamma_{s_{j}} \alpha$ onto $f^{*}\left(H \times M / \Gamma_{S_{j}}\right)$, and hence we have the trace map: $H^{1}\left(H / \Gamma_{S_{i}} \cap \alpha^{-1} \Gamma_{S_{j}} \alpha, H \times M / \Gamma_{S_{i}} \cap \alpha^{-1} \Gamma_{S_{j}} \alpha\right) \rightarrow H^{1}\left(H / \Gamma_{S_{j}}, H \times M / \Gamma_{S_{j}}\right)$.

Then $\mathfrak{T}^{\prime}(S x S)_{i j}$ and $\mathfrak{I}^{\prime \prime}(S x S)_{i j}$ commute with the natural isomorphisms of the group cohomologies to the sheaf cohomologies ((2.2.4)).

Proof. This follows from (2.2.4) and the transitivity of the trace map (cf. [1] XVII 6.2.3).

Q.E.D.

We define the endomorphism $\mathfrak{I}^{\prime \prime}(S x S)$ of $\oplus_{i=1}^{h} H^{1}\left(H / \Gamma_{S_{i}}, H \times M / \Gamma_{S_{i}}\right)$ by $\mathfrak{I}^{\prime \prime}(S x S)=\oplus_{i=1}^{h} \mathfrak{T}^{\prime \prime}(S x S)_{i j}$.

2.3. Before defining the étale cohomology groups in the next sub- 
section, we have to recall the theory of canonical models [17]. Let $\nu$ be the reduced norm from $B_{A+}^{\times}$to $F_{A}^{\times}$. For an element $x \in B_{A+}^{\times}$, we denote by $\sigma(x)$ the element of $\operatorname{Gal}\left(F_{a b} / F\right)$ which corresponds to $\nu(x)^{-1}$ by class field theory, where $F_{a b}$ denotes the maximal abelian extension of $F$. For a member $S$ of $\mathscr{Z}$, let $k_{S}$ be the subfield of $F_{a b}$ fixed under all $\sigma(s)$ with $s \in S ; k_{S}$ is a finite abelian extension of $F$. We first recall the main result of [17] restricted to our situation:

Theorem (2.3.1) (Shimura [17] 2.5). For each $S \in \mathscr{Z}$, we can attach a pair $\left(V_{S}, \varphi_{S}\right)$, where

(2.3.1.1) $V_{S}$ is a proper, smooth and geometrically irreducible curve over $k_{S}$. (2.3.1.2) Put $\Gamma_{S}=S \cap B_{+}^{\times}$. Then $\varphi_{s}$ is a $\Gamma_{s^{-}}$-invariant holomorphic map from $H$ onto $V_{S}(C)$ which induces a biholomorphic isomorphism of $H / \Gamma_{S}$ onto $V_{S}(C)$. Moreover, for $S, T \in \mathscr{Z}$ and $x \in B_{A+}^{\times}$such that $x S x^{-1} \subseteq T$, there is a morphism $J_{T S}(x): V_{S} \rightarrow V_{T}^{\sigma(x)}$ over $k_{S}$, where $V_{T}^{\sigma(x)}$ is the conjugate of $V_{T}$ by $\sigma(x)$ (i.e. $V_{T}^{\sigma(x)}$ is the base change of $V_{T}$ by Spec $\left.(\sigma(x))\right)$, and the system $\left\{V_{S}, \varphi_{S}, J_{T S}(x),(S, T \in\right.$ $\left.\left.\mathscr{Z}, x \in B_{A+}^{\times}\right)\right\}$satisfies the following conditions:

(2.3.1.3) $J_{S S}(s)$ is the identity if $s \in S$.

(2.3.1.4) $\quad J_{T S}(x)^{\sigma(y)} \circ J_{S R}(y)=J_{T R}(x y)$ if $x S x^{-1} \subseteq T$ and $y R y^{-1} \subseteq S$.

(2.3.1.5) $\quad J_{T S}(\alpha)\left[\varphi_{S}(z)\right]=\varphi_{T}(\alpha(z))$ if $\alpha \in B_{+}^{\times}$and $\alpha S \alpha^{-1} \subseteq T$.

There is one more important property which describes a certain reciprocity law at the isolated fixed points of $B_{+}^{\times}$, which also characterizes the system $\left\{V_{S}, \varphi_{S}, J_{T S}(x),\left(S, T \in \mathscr{Z}, x \in B_{A+}^{\times}\right)\right\}$. But we do not state it here because we will not need it explicitly in this paper.

We next recall the homomorphism $\tau$ defined in [17] 2.7. Let $L_{S}$ denote the field of rational functions of $V_{S}$ defined over $k_{S}$, and let $\mathscr{L}_{S}$ be the set of meromorphic functions on $H$ defined by $\left\{f \circ \varphi_{S} \mid f \in L_{S}\right\}$. We denote by $\mathscr{L}$ the union of all $\mathscr{L}_{S}(S \in \mathscr{Z})$. For an element $x \in B_{A+}^{\times}$, we put

$$
\left(f \circ \varphi_{S}\right)^{\tau(x)}=f^{\sigma(x)} \circ J_{S T}(x) \circ \varphi_{T} \text { for } f \in L_{S} \text { with } T=x^{-1} S x .
$$

This defines a homomorphism $\tau$ of $B_{A+}^{\times}$to $\operatorname{Aut}(\mathscr{L} \mid F)$, and $B_{A+}^{\times}$acts on $\mathscr{L}$ from the right by $\tau$. This $\tau$ has the following properties:

$$
\begin{gathered}
\tau(x)=\sigma(x) \text { on } F_{a b} . \\
h^{\tau(\alpha)}(z)=h(\alpha(z)) \text { for } \alpha \in B_{+}^{\times}, h \in \mathscr{L} \text { and } z \in H .
\end{gathered}
$$

TheOREM (2.3.5) '([17] 2.8). Let $\overline{F^{\times} B_{\infty+}^{\times}}$be the closure of $F^{\times} B_{\infty_{+}}^{\times}$in $B_{A_{+}}^{\times}$. If we topologize Aut $(\mathscr{L} \mid F)$ as in [17] 2.7, $\tau$ is continuous, $\tau\left(\overline{F^{\times} B_{\infty+}^{\times}}\right)=\{i d\}$, and $\tau$ induces a topological isomorphism of $B_{A_{+}}^{\times} / \overline{F^{\times} B_{\infty+}^{\times}}$onto $\operatorname{Aut}(\mathscr{L} / F)$. For $S \in \mathscr{Z}$, we also have $F^{\times} S=\left\{x \in B_{A+}^{\times} \mid \tau(x)=i d\right.$ on $\left.\mathscr{L}_{S}\right\}$. 
In the following, we identify $L_{S}$ with $\mathscr{L}_{S}$ by $\varphi_{S}$, and hence we consider $\tau(x)$ as an isomorphism of $L_{S}$ onto $L_{T}$ with $T=x^{-1} S x$. Under this convention, we denote by $[\tau(x)]=[\tau(x)]_{S T}$ the isomorphism of the scheme $V_{T}$ onto $V_{S}$ defined by $f \circ[\tau(x)]=f^{\tau(x)}$ for all $f \in L_{S}$. Note that $[\tau(x)]$ is not a $k_{S}$-morphism in general, but is an $F$-morphism if we consider $V_{S}$ and $V_{T}$ as $F$-schemes by means of the morphism: $\operatorname{Spec}\left(k_{S}\right) \rightarrow \operatorname{Spec}(F)$ which corresponds to the identity injection of $F$ into $k_{S}$. The relation between $[\tau(x)]$ and $J_{S T}(x)$ can be illustrated by the following commutative diagram:

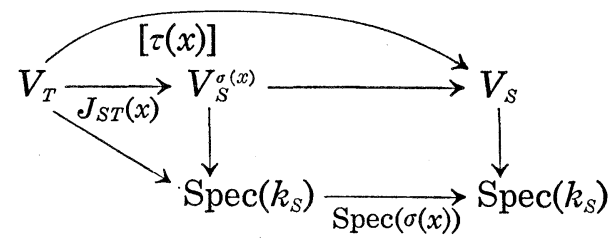

where the small square defines $V_{S}^{o(x)}$.

In the rest of this paper, we will also make the following convention: for $x S x^{-1} \subseteq T$ as in (2.3.1), we will use the same symbol $J_{T S}(x)$ to denote the $k_{T}$-morphism of $V_{S}$ onto $V_{T}^{\sigma(x)}$ such that the resulting pullback of a function $f \in L_{T}^{\sigma(x)} \subseteq L_{T}^{\sigma(x)} \otimes_{k_{T}} k_{S}$ is $f \circ J_{T S}(x)$. Let us fix $S, T \in \mathscr{Z}$, and suppose that $T$ is a normal subgroup of $S$. Then, by (2.5.3), $\tau$ induces an isomorphism of $S /\left(S \cap F^{\times}\right) T$ onto Gal $\left(L_{T} / L_{S}\right)$. Hence, for any $s \in S,[\tau(s)]=[\tau(s)]_{T T}$ is an automorphism of the scheme $V_{T}$ over $V_{S}$. It is obvious that $[\tau()]$ induces an isomorphism of $S /\left(S \cap F^{\times}\right) T$ onto $\operatorname{Aut}\left(V_{T} / V_{S}\right)$, and $S$ acts on $V_{T}$ from the left by $[\tau()]$.

2.4. In this subsection, we define certain étale sheaves and cohomology groups on $V_{S}$ following the method of Langlands [10]. Let $\rho$ be the representation introduced in 1.1. From now on, until the end of this paper, we assume that $\rho$ also satisfies the condition (2.1.6). Fix a member $S$ of $\mathscr{Z}$, and a maximal order $\mathfrak{o}$ of $B$ such that $S \subseteq B_{\infty+}^{\times} \times \prod \mathfrak{o}_{p}^{\times}$. We also assume, until 4.3, that $S$ satisfies the following condition:

(2.4.1) For any $x \in B_{A+}^{\times}, \Gamma_{S_{x}}$ has no non-trivial elliptic elements, where $S_{x}=$ $x^{-1} S x$.

Fix a prime number $l$. For each positive integer $n$, we put

$$
S_{n}=S \cap\left(B_{\infty+}^{\times} \times \prod_{\mathfrak{p} \backslash l} \mathfrak{o}_{\mathfrak{p}}^{\times} \times \prod_{\mathfrak{l} \mid l}\left(1+l^{n} \mathfrak{o}_{\mathfrak{l}}\right)\right),
$$

where the left product ranges over all the finite primes of $F$ which do not divide $l$, and the second one ranges over the rest of the finite primes of $F$. We then obtain a system of curves: $\cdots \rightarrow V_{S_{n+1}} \rightarrow V_{S_{n}} \rightarrow \cdots \rightarrow V_{S_{0}}=V_{S}$, where the morphisms are $J(1)$ (we will omit the subscript for $J$ when there is no 
fear of confusion). By the assumption (2.4.1), we see that $V_{S_{n}}$ is an étale Galois covering of $V_{S}$, and [ $\tau()$ ] induces an isomorphism of $S /\left(S \cap F^{\times}\right) S_{n}$ onto $\operatorname{Aut}\left(V_{S_{n}} / V_{S}\right)$, for each $n$.

Now let $X$ be the lattice in $\boldsymbol{R}^{m}$ as in (2.1.6), and consider the constant group scheme $V_{S_{n}} \times X / l^{n} X$ over $V_{S_{n}}$. We let $S$ act on this group scheme by $s \mapsto[\tau(s)] \times \rho(s)$ (cf. 2.1); this action is of course compatible with the action of $S$ on $V_{S_{n}}$. Since $S \cap F^{\times}$and $S_{n}$ act trivially on $X / l^{n} X$ by (1.4.5) and (2.1.6.2), the above action factors through $S /\left(S \cap F^{\times}\right) S_{n}$.

Definition (2.4.3). We denote by $F_{S, n}$ (or simply by $F_{n}$ when there is no fear of confusion) the quotient scheme $\left(S /\left(S \cap F^{\times}\right) S_{n}\right) \backslash V_{S_{n}} \times X / l^{n} X$.

$F_{n}$ is naturally equipped with the structure of a finite étale group over $V_{S}$, and we easily see that

$$
F_{n} \underset{V_{S}}{\times} V_{S_{n}} \cong V_{S_{n}} \times X / l^{n} X \text { canonically. }
$$

For $m \geqslant n$, we see that the natural injection $\tilde{i}_{m}^{n}: X / l^{n} X \rightarrow X / l^{m} X$ which sends $x$ mod $l^{n} X$ to $l^{m-n} x \bmod l^{m} X$ (resp. the canonical projection $\tilde{j}_{n}^{m}: X / l^{m} X$ $\left.\rightarrow X / l^{n} X\right)$ induces a monomorphism $i_{m}^{n}: F_{n} \rightarrow F_{m}$ (resp. an epimorphism $j_{n}^{m}: F_{m}$ $\rightarrow F_{n}$ ) of $V_{s}$-group schemes, because we could have formed the quotient from $V_{S_{m}}$ in defining $F_{n}$. Note that the base change of $i_{m}^{n}$ (resp. $j_{n}^{m}$ ) to $V_{S_{m}}$ is identified with $\tilde{i}_{m}^{n}$ (resp. $\tilde{j}_{n}^{m}$ ) by (2.4.4). We have thus defined an inductive system $\left(F_{n}, i_{m}^{n}\right)$ (resp. a projective system $\left(F_{n}, j_{n}^{m}\right)$ ) of finite étale groups over $V_{S}$. Considering $V_{S}$ as an $F$-scheme by $\operatorname{Spec}\left(k_{S}\right) \rightarrow \operatorname{Spec}(F)$, we can define the following two étale cohomology groups:

$$
\begin{aligned}
& \underset{\lim }{\rightarrow} H_{\dot{e} t}^{1}\left(V_{S} \underset{F}{\otimes} \bar{F}, F_{n} \underset{F}{\otimes} \bar{F}\right), \\
& \lim _{\leftarrow} H_{\dot{e} t}^{1}\left(V_{S} \underset{F}{\otimes} \bar{F}, F_{n} \underset{F}{\otimes} \bar{F}\right),
\end{aligned}
$$

where we have identified the group schemes $F_{n} \otimes_{F} \bar{F}$ and the étale sheaves represented by them, and $\lim \left(\right.$ resp. $\lim$ ) is taken relative to $\left\{i_{m}^{n}\right\}$ (resp. $\left\{j_{n}^{m}\right\}$ ). The Galois group Gal $(\bar{F} / \vec{F})$ acts on these cohomology groups from the right in a natural manner.

2.5. Let the notation be as in 2.4. In defining the cohomology groups, we considered the base changes of $V_{S}$ and $F_{n}$ from $F$ to $\bar{F}$. But the field of definition of $V_{S}$ is $k_{S}$, and we have $\operatorname{Gal}\left(k_{S} / F\right)=\left\{\sigma\left(x_{1}\right), \cdots, \sigma\left(x_{h}\right)\right\}$ with $\left\{x_{i}\right\}$ as in (1.4.2) by [17] 3.5. As before, we fix such a set of representatives $\left\{x_{i}\right\}$ satisfying the condition stated in 2.1. Then we have:

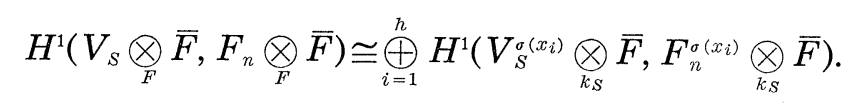


Put $S_{i, n}=x_{i}^{-1} S_{n} \dot{x}_{i}(1 \leqslant i \leqslant h)$. Let $[\tau(s)]^{\sigma\left(x_{i}\right)}$ be the automorphism of $V_{S_{n}}^{\sigma\left(x_{i}\right)}=$ $V_{S_{n}} \otimes_{k_{S_{n}}, \sigma\left(x_{i}\right)} k_{S_{n}}$ defined by $[\tau(s)] \times \operatorname{Spec}(\sigma(s))$. Then the following diagram commutes for all $s \in S$ :

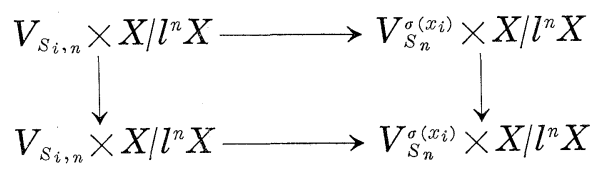

where two horizontal morphisms are $J\left(x_{i}\right) \times \rho\left(x_{i}\right)$, the left vertical morphism is $\left[\tau\left(x_{i}^{-1} s x_{i}\right)\right] \times \rho\left(x_{i}^{-1} s x_{i}\right)$, and the right vertical morphism is $[\tau(s)]^{\sigma\left(x_{i}\right)} \times \rho(s)$. Taking the quotient by the equivariant actions of $S_{i} /\left(S_{i} \cap F^{\times}\right) S_{i, n}$ and $S /\left(S \cap F^{\times}\right) S_{n}$, respectively, we have the following commutative diagram:

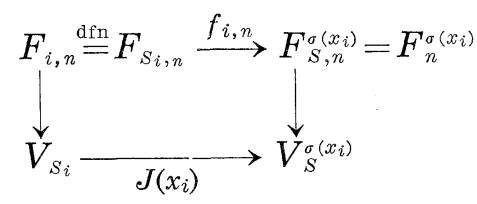

The above isomorphisms $\left\{f_{i, n}\right\}$ commute with $\left\{i_{m}^{n^{\sigma\left(x_{i^{\prime}}\right)}}\right\},\left\{j_{n}^{m^{\sigma\left(x_{i^{\prime}}\right.}}\right\}$ and the similar morphisms defined for $F_{i, n}$. This defines a natural isomorphism of cohomology groups:

$$
H^{1}\left(V_{S} \otimes_{F} \bar{F}, F_{n} \otimes_{F} \bar{F}\right) \cong \bigoplus_{i=1}^{h} H^{1}\left(V_{S_{i}} \otimes_{k_{S}} \bar{F}, F_{i, n} \bigotimes_{k_{S}} \bar{F}\right),
$$

which commutes with lim and lim. By the comparison theorem of the étale

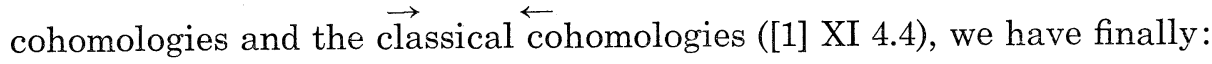

$$
\begin{aligned}
H^{1}\left(V_{S} \underset{F}{\otimes} \bar{F}, F_{n} \underset{F}{\otimes} \bar{F}\right) & \cong \bigoplus_{i=1}^{h} H^{1}\left(H \mid \Gamma_{S_{i}}, H \times\left(X \mid l^{n} X\right) / \Gamma_{S_{i}}\right) \\
& \cong \bigoplus_{i=1}^{h} H^{1}\left(\bar{\Gamma}_{S_{i}}, X \mid l^{n} X\right)
\end{aligned}
$$

which commutes with lim and lim.

Put $X_{i}=\rho\left(x_{i}^{-1}\right)\left(X \overrightarrow{\otimes_{Z}}\left(\boldsymbol{R} \times \prod_{p}^{\leftarrow} \boldsymbol{Z}_{p}\right)\right) \cap \boldsymbol{Q}^{m}$. Then $X_{i}$ is a $Z$-lattice in $\boldsymbol{Q}^{m}$ which is $\Gamma_{S_{i}}$-stable. Since $x_{i} \in A_{l}, X_{i} / l^{n} X_{i}$ are canonically isomorphic to $X / l^{n} X$ as $\Gamma_{S_{i}}$-modules. From the short exact sequence: $0 \rightarrow X_{i} \stackrel{l^{n}}{\rightarrow} X_{i} \rightarrow X_{i} / l^{n} X_{i} \rightarrow 0$, we have the exact sequence:

$$
0 \rightarrow H^{1}\left(\bar{\Gamma}_{S_{i}}, X_{i}\right) \otimes \boldsymbol{Z} / l^{n} \boldsymbol{Z} \rightarrow H^{1}\left(\bar{\Gamma}_{S_{i}}, X_{i} / l^{n} X_{i}\right) \rightarrow{ }_{l^{n}} H^{2}\left(\bar{\Gamma}_{S_{i}}, X_{i}\right) \rightarrow 0
$$

where ${ }_{l^{n}} G$ denotes the kernel of the multiplication by $l^{n}$ for an abelian group $G$. Since the torsion subgroup of $H^{2}\left(\bar{\Gamma}_{s_{i}}, X_{i}\right)$ is finite (cf. [18] Prop. 8.2), and the image of $H^{1}\left(\bar{\Gamma}_{S_{i}}, X_{i}\right)$ in $H^{1}\left(\bar{\Gamma}_{S_{i}}, \boldsymbol{R}^{m}\right)$ is a lattice by [18] Prop.8.6, we have: 
(2.5.6) The maximal divisible subgroup $D$ of $\lim H^{1}\left(V_{S} \otimes_{F} \bar{F}, F_{n} \otimes_{F} \bar{F}\right)$ is isomorphic to $\left(\boldsymbol{Q}_{l} / \boldsymbol{Z}_{l}\right)^{2 d}$, and

$$
\lim _{\leftarrow} H^{1}\left(V_{S} \underset{F}{\otimes} \bar{F}, F_{n} \underset{F}{\otimes} \bar{F}\right) \underset{Z_{l}}{\otimes} \boldsymbol{Q}_{l} \cong \boldsymbol{Q}_{l}^{2 d},
$$

with $d=\operatorname{dim}_{c} \subseteq(S, \rho)$. It is obvious that the representations $\operatorname{Gal}(\bar{F} / F) \rightarrow$ $\operatorname{Aut}(D) \cong G L_{2 d}\left(Z_{l}\right)$ and $\operatorname{Gal}(\bar{F} / F) \rightarrow \operatorname{Aut}_{Q_{l}}\left(\lim _{\leftarrow} H^{1}\left(V_{S} \otimes_{F} \bar{F}, F_{n} \otimes_{F} \bar{F}\right) \otimes_{Z_{l}} \boldsymbol{Q}_{l}\right) \cong$ $G L_{2 d}\left(\boldsymbol{Q}_{l}\right)$ are equivalent.

Definition (2.5.8). We denote by $\varphi_{S, o}$ (the equivalence class of ) one of these equivalent representations.

2.6. Lemma (2.6.1). The notation being as in 2.4, let $x$ be an element of $A_{l} \cap V(S)$ such that the p-components of $x$ are contained in $\mathfrak{p}_{p}^{\times}$for all $\mathfrak{p} \mid L(S)$ (cf. (1.5.1) and 2.1). For non-negative integers $n$, put $U_{n}=S_{n} \cap x^{-1} S_{n} x$, where $S_{0}=S$. We write $U$ for $U_{0}$. Then we have $k_{S_{n}}=k_{U_{n}}$, and $U_{n}=U \cap S_{n}=$ $U \cap x^{-1} S_{n} x$ for all $n$. Also we have that the natural maps: $U /\left(U \cap F^{\times}\right) U_{n} \rightarrow$ $S /\left(S \cap F^{\times}\right) S_{n}$ and $x\left(U /\left(U \cap F^{\times}\right) U_{n}\right) x^{-1} \rightarrow S /\left(S \cap F^{\times}\right) S_{n}$ are isomorphisms for all $n$.

Proof. Since $\nu\left(U_{n}\right)=\nu\left(S_{n}\right)$, the first assertion is obvious. The second assertion can be checked easily, and the injectivity of the above maps follows from this. We prove the surjectivity by showing that the groups in question have the same order. By the assumption on $x$, we have $S=U S_{n}$, and hence $\left[S: S_{n}\right]=\left[U: U_{n}\right]$. But $\left[\left(S \cap F^{\times}\right) S_{n}: S_{n}\right]=\left[S \cap F^{\times}: S_{n} \cap F^{\times}\right]=$ $\left[\left(U \cap F^{\times}\right) U_{n}: U_{n}\right]$, and hence our assertion follows.

Q.E.D.

Fix an element $x$ as above, and consider the commutative diagram:

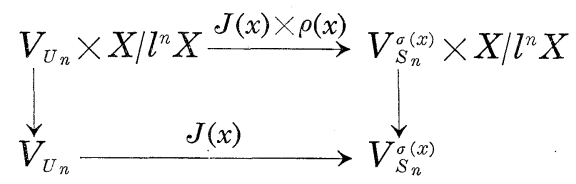

As in (2.5.1), we have $(J(x) \times \rho(x))([\tau(u)] \times \rho(u))=\left(\left[\tau\left(x u x^{-1}\right)\right]^{\sigma(x)} \times \rho\left(x u x^{-1}\right)\right)(J(x)$ $\times \rho(x))$ for all $u \in U$. Taking the quotient by the equivariant actions of $x\left(U /\left(U \cap F^{\times}\right) U_{n}\right) x^{-1} \cong S /\left(S \cap F^{\times}\right) S_{n}$, we obtain the following cartesian diagram:

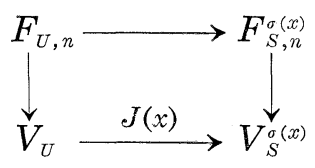

Also from the diagram: 


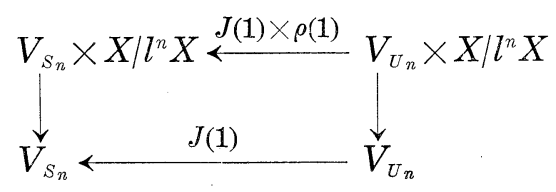

we obtain, by a similar procedure, the following cartesian diagram:

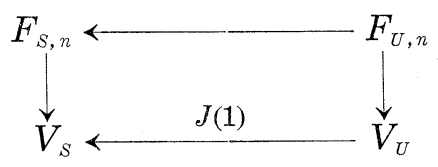

From (2.6.3) (resp. (2.6.5)), we obtain a canocnical isomorphism of the étale sheaf $J(x)^{*} F_{S, n}^{o(x)}\left(\right.$ resp. $\left.J(1)^{*} F_{S, n}\right)$ to $F_{U, n}$ (resp. $F_{U, n}$ ). For brevity, we write $\bar{V}_{S}$ (resp. $\bar{F}_{S, n}$ ) for $V_{S} \otimes_{F} \bar{F}$ (resp. $F_{S, n} \otimes_{F} \bar{F}$ ) in the following. We also use the similar notation for $V_{U}$ and $V_{S}^{\sigma(x)}$ etc..

Definiton (2.6.6). Using the notation as above, we define the endomorphism $\mathfrak{T}^{*}(S x S)_{n}$ of $H^{1}\left(\bar{V}_{S}, \bar{F}_{S, n}\right)$ as the composite of the following two maps:

i) the cononical map: $H^{1}\left(\bar{V}_{S}, \bar{F}_{S, n}\right) \rightarrow H^{1}\left(\bar{V}_{U}, \bar{F}_{U, n}\right)$,

ii) the trace map: $H^{1}\left(\bar{V}_{U}, \bar{F}_{U, n}\right) \rightarrow H^{1}\left(\bar{V}_{S}^{\sigma(x)}, \bar{F}_{S, n}^{\sigma(x)}\right)=H^{1}\left(\bar{V}_{S}, \bar{F}_{S, n}\right)$ (cf. [1] IX 5 , XVII 6.2), for $x \in B_{A^{+}}^{\times}$as in (2.6.1).

It is obvious that $\mathfrak{I}^{*}(S x S)_{n}$ commute with $\lim _{\rightarrow}\left(\right.$ resp. $\left.\lim _{\leftarrow}\right)$ relative to $\left\{i_{m}^{n}\right\}$ (resp. $\left\{j_{n}^{m}\right\}$ ).

Proposition (2.6.7). Let $\varphi: H^{1}\left(\bar{V}_{S}, \bar{F}_{S, n}\right) \rightarrow \oplus_{i=1}^{n} H^{1}\left(H / \Gamma_{S_{i}}, H \times\left(X / l^{n} X\right) / \Gamma_{S_{i}}\right)$ be the isomorphism in (2.5.4). Then we have $\varphi \circ \mathfrak{T}^{*}(S x S)_{n}=\mathfrak{I}^{\prime \prime}(S x S)_{n} \circ \varphi$ for all $x \in B_{A+}^{\times}$satisfying the condition stated in (2.6.1), where $\mathbb{I}^{\prime \prime}(S x S)_{n}$ is the operator $\mathfrak{T}^{\prime \prime}(S x S)$ defined in 2.2 for $M=X \mid l^{n} X$.

Proof. $\mathfrak{T}^{*}(S x S)_{n}$ is the direct sum of the operators $\mathfrak{T}^{*}(S x S)_{n, i j}$ :

$$
H^{1}\left(V_{S}^{\sigma\left(x_{i}\right)} \otimes_{k_{S}} \bar{F}, F_{S, n}^{\sigma\left(x_{i}\right)} \underset{k_{S}}{\otimes} \bar{F}\right) \longrightarrow H^{1}\left(V_{S}^{\sigma\left(x_{j}\right)} \underset{k_{S}}{\otimes} \bar{F}, F_{S, n}^{\sigma\left(x_{j}\right)} \bigotimes_{k_{S}} \bar{F}\right)
$$

with $j$ determined by the relation $x x_{i}=s x_{j} \alpha$ as before. For each $i$, put $U_{i, n}$ $=x_{i}^{-1} U_{n} x_{i}$, and consider the commutative diagram:

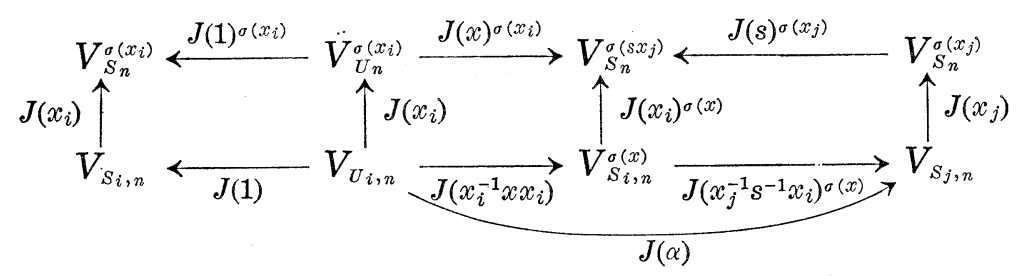


and a system of constant group schemes over it:

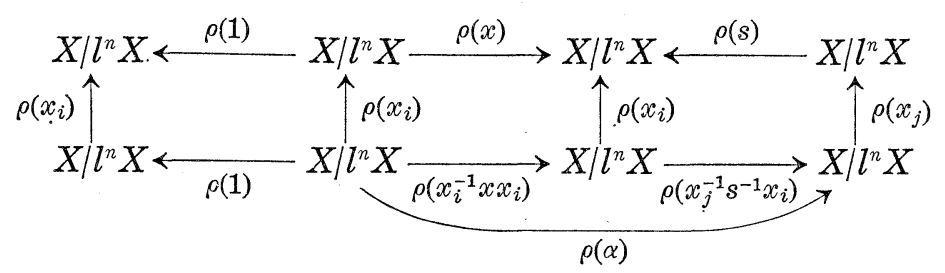

As above and 2.5, taking the quotients by the equivariant actions of $U /\left(U \cap F^{\times}\right) U_{n} \cong S /\left(S \cap F^{\times}\right) S_{n} \cong x\left(U /\left(U \cap F^{\times}\right) U_{n}\right) x^{-1}$ and their conjugates, we obtain the following commutative diagram:

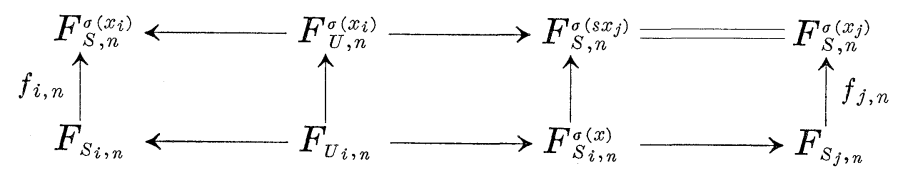

over the diagram of the curves:

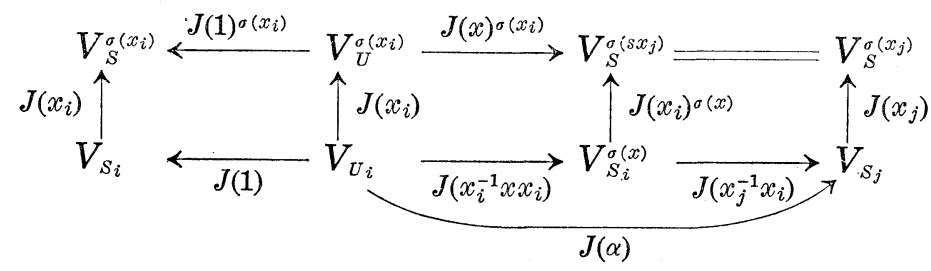

Since $\Gamma_{U_{i}}=\Gamma_{S_{i}} \cap \alpha^{-1} \Gamma_{S_{j}} \alpha$, we obtain our assertion by the comparison theorem.

Q.E.D.

\section{$\S 3$. The congruence relation}

3.1. The goal of this section is the congruence relation for our representation $\varphi_{S, \rho}((3.8 .5))$. We begin with a preparatory result in this subsection. Let the notation be as in 2.4. By (2.4.4), we have the canonical map:

$$
\omega_{n}: H^{1}\left(\bar{V}_{s}, \bar{F}_{n}\right) \longrightarrow H^{1}\left(\bar{V}_{S_{n}}, X / l^{n} X\right),
$$

where we use the abbreviated notation $\bar{V}_{S_{n}}$ instead of $V_{S_{n}} \otimes_{F} \bar{F}$ as in 2.6, and $X / l^{n} X$ instead of $X / l^{n} X \times \bar{V}_{s_{n}}$. For $n \leqslant m$, we have the natural map $H^{1}\left(\bar{V}_{s}, \bar{F}_{n}\right)$ $\rightarrow H^{1}\left(\bar{V}_{S}, \bar{F}_{m}\right)$ which is induced by $i_{m}^{n}$. We also have the map $H^{1}\left(\bar{V}_{S_{n}}, X / l^{n} X\right)$ $\rightarrow H^{1}\left(\bar{V}_{S_{m}}, X / l^{n} X\right) \rightarrow H^{1}\left(\bar{V}_{S_{m}}, X / l^{m} X\right)$, where the first map is the canonical map, and the latter one is induced by $\tilde{i}_{m}^{n}$. They are of course compatible with the map (3.1.1). Taking the inductive limits relative to these maps, we obtain: 


$$
\text { (1) } \lim _{\rightarrow} H^{1}\left(\bar{V}_{S}, \bar{F}_{n}\right) \longrightarrow \lim _{\rightarrow} H^{1}\left(\bar{V}_{S_{n}}, X / l^{n} X\right) .
$$

TheOREM (3.1.3) (Shimura). The kernel of the above map $\omega$ is finite.

Since the proof is not short, we divide it in several steps. We first interpret the problem in an elementary language. Let $B_{A+}^{\times}=\bigcup_{k, i} S_{n} s_{k} x_{i} B_{+}^{\times}=$ $\bigcup_{I=(k, i)} S_{n} y_{I} B_{+}^{\times}$be a disjoint decomposition with $s_{k} \in S$ and $y_{I}=s_{k} x_{i}$ when $I=$ $(k, i)$. We may suppose that $s_{1}=1$. Putting $S_{I, n}=y_{I}^{-1} S_{n} y_{I}$ for each $I$, we have the natural isomorphism $H^{1}\left(\bar{V}_{S_{n}}, X / l^{n} X\right) \cong \oplus_{I} H^{1}\left(\bar{\Gamma}_{S_{I, n}}, X / l^{n} X\right)$ by (2.5.4). For each $i(1 \leqslant i \leqslant h)$, let $\omega_{i, n}$ be the restriction: $H^{1}\left(\bar{\Gamma}_{S_{i}}, X / l^{n} X\right) \rightarrow H^{1}\left(\bar{\Gamma}_{s_{i}, n}\right.$, $\left.X / l^{n} X\right)$. Then it is easy to see that this corresponds, via (2.5.3) and (2.5.4), to the map: $H^{1}\left(V_{S_{i}} \otimes_{k_{S}} \bar{F}, F_{i, n} \otimes_{k_{S}} \bar{F}\right) \rightarrow H^{1}\left(V_{S_{i}, n} \otimes_{k_{S_{n}}} \bar{F}, X / l^{n} X\right)=H^{1}\left(V_{S_{I, n}} \otimes_{k_{S_{n}}} \bar{F}\right.$, $\left.X / l^{n} X\right)$ with $I=(1, i)$ for summands in (3.1.1), and the inductive limit of the latter can be identified with the natural inductive limit of the former. Therefore to prove (3.1.3), it is sufficient to prove the following.

THEOREM (3.1.4). The kernel of $\lim _{\rightarrow} \omega_{i, n}=\omega_{i}$ is finite for each $i$.

Let $\mathfrak{o}$ be a maximal order of $B$ such that $S \subseteq B_{\infty_{+}}^{\times} \times \prod \mathfrak{o}_{p}^{\times}$as before, and put $\mathfrak{o}_{i}=x_{i}^{-1}\left(B_{\infty} \times \prod \mathfrak{o}_{\mathfrak{p}}\right) x_{i} \cap B$. Then $\mathfrak{o}_{i}$ is also a maximal order of $B$, and $\Gamma_{S_{i}}$ is a congruence subgroup of $\mathfrak{b}_{i+}^{\times}=\left\{\gamma \in \mathfrak{o}_{i}^{\times} \mid \nu(\gamma)\right.$ is totally positive $\}$. We also note that $\Gamma_{S_{i}, n}=\left\{\gamma \in \Gamma_{S_{i}} \mid \gamma-1 \in l^{n} \mathfrak{D}_{i}\right\}$.

3.2. Let us now take a maximal order o of $B$, and a lattice $X$ in $\boldsymbol{Q}^{m}$ satisfying (2.1.6) for this $\mathfrak{D}$. Let $\Gamma$ be a congruence subgroup of $\mathfrak{o}_{+}^{\times}$such that $\rho\left(\Gamma \cap F^{\times}\right)=\{1\}$, and put $\Gamma_{n}=\left\{\gamma \in \Gamma \mid \gamma-1 \in l^{n} \mathfrak{o}\right\}$ for $n \geqslant 0$. We will show, in the next subsection, the following assertion:

(3.2.1) The kernel of the natural map $\xi: \lim _{\rightarrow} H^{1}\left(\bar{\Gamma}, X \mid l^{n} X\right) \rightarrow \underset{\rightarrow}{\lim } H^{1}\left(\bar{\Gamma}_{n}, X \mid l^{n} X\right)$ is finite,

which will conclude the proof of (3.1.3). We first reduce the problem to subgroups of special type.

LEMMA (3.2.2). Let $\Gamma \supseteq \Gamma^{\prime}$ be two congruence subgroups of $\mathfrak{b}_{+}^{\times}$such that $\rho\left(\Gamma \cap F^{\times}\right)=\{1\}$. If (3.2.1) holds for $\Gamma^{\prime}$, then it also holds for $\Gamma$.

Proof. From the exact sequence similar to (2.5.5), we have a natural injective map $\eta: H^{1}(\bar{\Gamma}, X) \otimes \boldsymbol{Q}_{l} / \boldsymbol{Z}_{l} \longrightarrow \lim H^{1}\left(\bar{\Gamma}, X / l^{n} X\right)$ and a similar map $\eta^{\prime}$ for $\Gamma^{\prime}$. Consider the following commutative diagram:

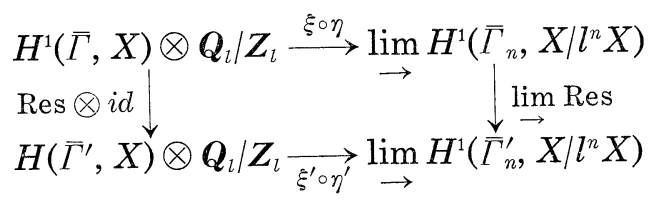


where $\xi^{\prime}$ is the map defined similarly as $\xi$ for $\Gamma^{\prime}$. By our assumption, the kernel of $\xi^{\prime} \circ \eta^{\prime}$ is finite. Since the cokernels of $\eta$ and $\eta^{\prime}$ are finite, it is enough to show that the kernel of the left vertical map is finite. But since $H^{1}(\bar{\Gamma}, X)$ is finitely generated, it is enough to show that the restriction: $H^{1}(\bar{\Gamma}, X) \otimes \boldsymbol{R} \rightarrow H^{1}\left(\bar{\Gamma}^{\prime}, X\right) \otimes \boldsymbol{R}$ is injective. By [18] Prop. 8.6, this can be identified with the restriction: $H^{1}\left(\bar{\Gamma}, \boldsymbol{R}^{m}\right) \rightarrow H^{1}\left(\bar{\Gamma}^{\prime}, \boldsymbol{R}^{m}\right)$. Via the isomorphism (2.1.3), this can be identified with the natural inclusion: $\widetilde{S}(\Gamma, \rho) \longrightarrow \widetilde{S}\left(\Gamma^{\prime}, \rho\right)$.

Q.E.D.

By this lemma, we may restrict ourselves to the principal congruence subgroups $\Gamma(N)=\left\{\gamma \in \mathfrak{D}_{+}^{\times} \mid 1-\gamma \in N\right.$ o $\}$ with positive integers $N$.

LEMMA (3.2.3) (cf. [15] 3.3). Put $\mathfrak{D}^{u}=\{\alpha \in \mathfrak{D} \mid \nu(\alpha)=1\}$, and $\Gamma^{\prime}(N)=\left\{\gamma \in \mathfrak{D}^{u} \mid\right.$ $\gamma-1 \in N_{\mathfrak{D}\}}$. Then there is a subgroup $E$ of the unit group $\mathfrak{x}_{F}^{\times}$of the integer ring $\mathfrak{x}_{F}$ of $F$ such that $E \Gamma^{\prime}(N)$ is a congruence subgroup of $\mathfrak{o}_{+}^{\times}$which is contained in $\Gamma(N)$.

Proof. Let $E_{+}$be the group of totally positive units of $F$, and put $E=$ $\left\{\varepsilon \in E_{+} \mid 1-\varepsilon \in N \mathfrak{r}_{F}\right\}$. Then it is easy to see that $E \Gamma^{\prime}(N)=\left\{\gamma \in \Gamma(N) \mid \nu(\gamma) \in E^{2}\right\}$. By a theorem of Chevalley [2], there exists a positive integer $m$ such that $\{\varepsilon \in E \mid \varepsilon \equiv 1 \bmod m\} \subseteq E^{2}$, and hence we see that $\Gamma(m N) \subseteq E \Gamma^{\prime}(N) . \quad$ Q.E.D.

Note that, taking $N$ sufficiently large, we may suppose that $F^{\times} \cap \Gamma^{\prime}(N)$ $=\{1\}$ in proving (3.2.1).

By the inflation-restriction sequence, the kernel of the restriction: $H^{1}\left(\bar{\Gamma}, X / l^{n} X\right) \rightarrow H^{1}\left(\bar{\Gamma}_{n}, X / l^{n} X\right)$ is isomorphic to $H^{1}\left(\bar{\Gamma} / \bar{\Gamma}_{n}, X / l^{n} X\right)$. Hence to prove (3.2.1), it is sufficient to show that $\left|H^{1}\left(\bar{\Gamma} / \bar{\Gamma}_{n}, X / l^{n} X\right)\right| \leqslant M$ for all $n$ with a constant $M$ which does not depend on $n$, for the groups $\Gamma=E \Gamma^{\prime}(N)$ as above. Since the natural map: $\Gamma^{\prime}(N) / \Gamma^{\prime}(N)_{n} \rightarrow \bar{\Gamma} / \bar{\Gamma}_{n}$ is surjective, and its kernel stabilizes $X / l^{n} X$, the inflation maps $H^{1}\left(\bar{\Gamma} / \bar{\Gamma}_{n}, X / l^{n} X\right)$ injectively into $H^{1}\left(\Gamma^{\prime}(N) / \Gamma^{\prime}(N)_{n}, X / l^{n} X\right)$, and hence our problem is reduced to the subgroups $\Gamma^{\prime}(N)$ for $N$ large.

3.3. We change the notation and put $\Gamma=\left\{\gamma \in \mathfrak{D}^{u} \mid \gamma-1 \in N \mathfrak{o}\right\}$, and $\Gamma_{n}=$ $\left\{\gamma \in \Gamma \mid \gamma-1 \in l^{n} \mathfrak{D}\right\}$ with $N$ sufficiently large. Take and fix a finite extension $k$ of $\boldsymbol{Q}$ satisfying the following three conditions:

(3.3.1) $\quad k$ is totally real, and linearly disjoint with $F$ over $\boldsymbol{Q}$.

(3.3.2) Any prime factor of $l N N_{F / Q}(D(B / F))$ splits completely in $k$.

(3.3.3) $\quad[k: \boldsymbol{Q}] \geqslant 2$.

Such a $k$ of course exists. By the first two conditions, $B^{\prime}=B \otimes_{Q} k$ is a division quaternion algebra over the totally real field $K=F \otimes_{Q} k$. Since $\rho$ is a homomorphism of algebraic groups over $\boldsymbol{Q}$, we have, by taking the $k$-valued points: 


$$
\rho_{k}: B^{\prime \times}=(B \underset{Q}{\otimes} k)^{\times} \longrightarrow G L_{m}(k) .
$$

Put $\mathfrak{D}^{\prime}=\mathfrak{D} \otimes_{Z} \mathfrak{x}_{k}$ and $X^{\prime}=X \otimes_{Z} \mathfrak{x}_{k}$. Take a prime $\mathfrak{l}$ of $k$ which divides $l$, and put $\mathfrak{b}_{\mathfrak{l}}^{\prime}=\mathfrak{D} \otimes_{Z} \mathfrak{x}_{k \mathfrak{l}}$ and $X_{\mathfrak{l}}^{\prime}=X \otimes_{Z} \mathfrak{x}_{k \mathfrak{l}}$, where $\mathfrak{r}_{k \mathfrak{l}}$ is the $\mathfrak{l}$-adic completion of $\mathfrak{r}_{k}$. We also put $\mathfrak{D}^{\prime \prime}=\mathfrak{v}_{\mathfrak{r}}^{\prime} \cap B^{\prime}$ and $X^{\prime \prime}=X_{\mathfrak{r}}^{\prime} \cap\left(X \otimes_{Z} k\right)$.

LEMMA (3.3.5). Using the notation as above, we have

(3.3.5.1) For any $\alpha \in \mathfrak{D}^{\prime \prime} \cap B^{\prime \times}, X^{\prime \prime}$ is $\rho_{k}(\alpha)$-stable.

(3.3.5.2) For any $\alpha \in \mathfrak{D}^{\prime \prime} \cap B^{\prime \times}$ such that $\alpha-1 \in \mathfrak{I}^{n} \mathfrak{o}^{\prime \prime}$, we have $\left(\rho_{k}(\alpha)-\rho_{k}(1)\right) X^{\prime \prime}$ $\subseteq \mathfrak{l}^{n} X^{\prime \prime}$.

Proof. For any $\alpha \in \mathfrak{o}_{\mathfrak{l}}^{\prime} \cap\left(B^{\prime} \otimes_{k} k_{\mathfrak{l}}\right)^{\times}$, we have $\rho_{k}(\alpha) X_{\mathfrak{l}}^{\prime} \subseteq X_{\mathfrak{l}}^{\prime}$ since $\mathfrak{l}$ is of degree one. If $\alpha$ is as in (3.3.5.1), then we have $\rho_{k}(\alpha) X^{\prime \prime} \subseteq X_{\mathfrak{\imath}}^{\prime} \cap\left(X \otimes_{Z} k\right)=X^{\prime \prime}$, which proves the first assertion. The second assertion can be proved similarly.

Q.E.D.

Note that the natural map: $X / l^{n} X \rightarrow X^{\prime \prime} / \mathfrak{L}^{n} X^{\prime \prime}$ is an isomorphism.

Let $N=\prod_{i=1}^{m} p_{i}^{e_{i}}$ be the prime decomposition of $N$, and choose one prime $\mathfrak{p}_{i}$ above $p_{i}$ in $k$ for each $i(1 \leqslant i \leqslant m)$. Put $\Gamma^{\prime}=\left\{\gamma \in \mathfrak{D}^{\prime} \mid \nu_{B^{\prime} / K}(\gamma)=1, \gamma-1 \in \mathfrak{p}_{i}^{e_{i}} \mathfrak{D}^{\prime}\right.$ for $1 \leqslant i \leqslant m\}$, and $\Gamma_{n}^{\prime}=\left\{\gamma \in \Gamma^{\prime} \mid \gamma-1 \in \mathfrak{I}^{n} \mathfrak{D}^{\prime}\right\}$. By the above lemma, $X^{\prime \prime} / \mathfrak{l}^{n} X^{\prime \prime}$ is a $\Gamma^{\prime} \mid \Gamma_{n}^{\prime}$-module by $\rho_{k}$. By the injection of $B$ into $B^{\prime}=B \otimes_{Q} k$, which sends $x$ to $x \otimes 1$, we may identify $B$ with a subring of $B^{\prime}$. It is easy to see that

$$
B^{\times} \cap \Gamma^{\prime}=\Gamma \quad \text { and } \quad B^{\times} \cap \Gamma_{n}^{\prime}=\Gamma_{n} .
$$

It is obvious that the action of $\Gamma / \Gamma_{n}$ on $X^{\prime \prime} / \mathfrak{l}^{n} X^{\prime \prime} \cong X / l^{n} X$ through the natural map $\Gamma / \Gamma_{n} \prec \Gamma^{\prime} / \Gamma_{n}^{\prime}$ and $\rho_{k}$ coincides with the original action by $\rho$.

Lemma (3.3.7). The natural map $\Gamma / \Gamma_{n} \longleftrightarrow \Gamma^{\prime} / \Gamma_{n}^{\prime}$ is an isomorphism.

Proof. We have to prove the surjectivity. Put $B^{\prime u}=\left\{\alpha \in B^{\prime \times} \mid \nu_{B^{\prime} / K}(\alpha)=\right.$ 1\}. Consider the subgroups of the adelization $B_{A}^{\prime u}$ of $B^{\prime u}$ defined by $S^{\prime}=B_{\infty}^{\prime u}$ $\times \prod_{q \neq \mathfrak{q}_{i}}\left\{x_{q} \in \mathfrak{O}_{q}^{\prime} \mid \nu\left(x_{q}\right)=1\right\} \times \prod_{i=1}^{m}\left\{x_{\mathfrak{q}_{i}} \in \mathfrak{D}_{\mathfrak{p}_{i}}^{\prime} \mid \nu\left(x_{\mathfrak{p}_{i}}\right)=1, x_{\mathfrak{p}_{i}}-1 \in \mathfrak{p}_{i}^{e_{i} \mathfrak{O}_{\mathfrak{p}_{i}}^{\prime}}\right\}$ and $S_{n}^{\prime}=\left\{x \in S^{\prime} \mid\right.$

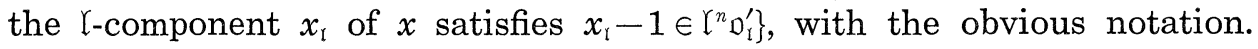
Then we have $\Gamma^{\prime}=B^{\prime \times} \cap S^{\prime}$ and $\Gamma_{n}^{\prime}=B^{\prime \times} \cap S_{n}^{\prime}$. By the approximation theorem (cf. [17] 3.4), we have $S_{n}^{\prime} B^{\prime u}=B_{A}^{\prime u}$, and hence the natural inclusion $\Gamma^{\prime} \mid \Gamma_{n}^{\prime} \hookrightarrow$ $S^{\prime} / S_{n}^{\prime}$ is an isomorphism. This shows that the index $\left[\Gamma^{\prime}: \Gamma_{n}^{\prime}\right]$ is equal to the index of $\left\{x \in \mathfrak{D}_{\mathfrak{l}}^{\prime} \mid \nu(x)=1, x-1 \in \mathfrak{I}^{A} \mathfrak{D}_{\mathfrak{l}}^{\prime}\right\}$ in $\left\{x \in \mathfrak{b}_{\mathfrak{l}}^{\prime} \mid \nu(x)=1, x-1 \in \mathfrak{I}^{e} \mathfrak{l}_{\mathfrak{l}}^{\prime}\right\}$ with $e_{l}=$ $\operatorname{ord}_{l}(N)$ and $A_{n}=\operatorname{Max}\left(e_{l}, n\right)$. Since we assumed (3.3.2), the same computation for $\Gamma$ and $\Gamma_{n}$ yields $\left[\Gamma^{\prime}: \Gamma_{n}^{\prime}\right]=\left[\Gamma: \Gamma_{n}\right]$.

Q.E.D.

Corollary (3.3.8). $\quad H^{1}\left(\Gamma / \Gamma_{n}, X / l^{n} X\right) \cong H^{1}\left(\Gamma^{\prime} / \Gamma_{n}^{\prime}, X^{\prime \prime} / \mathfrak{L}^{n} X^{\prime \prime}\right)$ for all $n$.

Now the inflation maps $H^{1}\left(\Gamma^{\prime} \mid \Gamma_{n}^{\prime}, X^{\prime \prime} / \mathfrak{L}^{n} X^{\prime \prime}\right)$ injectively into $H^{1}\left(\Gamma^{\prime}\right.$, $\left.X^{\prime \prime} / \mathfrak{L}^{n} X^{\prime \prime}\right)$. From the exact sequence: $0 \rightarrow \mathfrak{l n}^{n} X^{\prime \prime} \rightarrow X^{\prime \prime} \rightarrow X^{\prime \prime} / \mathfrak{L}^{n} X^{\prime \prime} \rightarrow 0$, we have the exact sequence: $H^{1}\left(\Gamma^{\prime}, X^{\prime \prime}\right) \rightarrow H^{1}\left(\Gamma^{\prime}, X^{\prime \prime} / \mathfrak{l}^{n} X^{\prime \prime}\right) \stackrel{\psi}{\rightarrow} H^{2}\left(\Gamma^{\prime}, \mathfrak{l}^{n} X^{\prime \prime}\right) \cong H^{2}\left(\Gamma^{\prime}, X^{\prime \prime}\right)$. 
Since the middle group is torsion, it is mapped into the torsion subgroup $H^{2}\left(\Gamma^{\prime}, X^{\prime \prime}\right)_{\text {tors }}$ of $H^{2}\left(\Gamma^{\prime}, X^{\prime \prime}\right)$. But the groups $H^{i}\left(\Gamma^{\prime}, X^{\prime \prime}\right)$ are finitely generated over $\mathfrak{r}_{k i} \cap k$ for all $i$, by Serre [14] Prop. 18, and hence the order of the image of $\psi$ does not exceed that of $H^{2}\left(\Gamma^{\prime}, X^{\prime \prime}\right)_{\text {tors }}$ which is finite and independent of $n$. On the other hand, we see, by an argument similar to that of [18] 8.4, that the kernel of the natural map: $H^{1}\left(\Gamma^{\prime}, X^{\prime \prime}\right) \rightarrow H^{1}\left(\Gamma^{\prime}, X^{\prime \prime} \otimes_{Z_{(l)}} C\right)$ is torsion, and hence finite, where $\boldsymbol{Z}_{(l)}=\boldsymbol{Z}_{l} \cap \boldsymbol{Q}$. By the same reason as in the proof of (2.2.4), the latter group is isomorphic to the sheaf cohomology $H^{1}\left(H^{d} / \Gamma^{\prime}\right.$, $\left.H^{d} \times\left(k^{m} \otimes_{Q} C\right) / \Gamma^{\prime}\right)$ with $d=[k: Q]$, where $\Gamma^{\prime}$ acts on $k^{m} \otimes_{Q} C$ through its action on the first factor by $\rho_{k}$. But this action of $\Gamma^{\prime}$ is extendable to a rational representation:

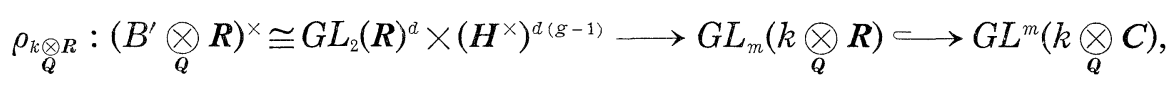

and $\Gamma^{\prime}$ can be considered as an irreducible discrete subgroup of $S L_{2}(\boldsymbol{R})^{d}$. Therefore the above sheaf cohomology group vanishes by Matsushima, Shimura [12] Th. 7.1 because we assumed that $d \geqslant 2$. This together with (3.3.8) concludes that there exists a constant $M$ which is independent of $n$ such that $\left|H^{1}\left(\Gamma\left|\Gamma_{n}, X\right| l^{n} X\right)\right| \leqslant M$ for all $n \geqslant 0$, and our proof of (3.1.3) is now complete.

3.4. Shimura's congruence relation and Morita's result. We quote here Shimura's congruence relation for modular correspondences on $V_{S}$ and Morita's good reduction theorem for Shimura curves. They are key to the whole of our theory as we will see below.

Take $S, T \in \mathscr{Z}$ and $x \in B_{A+}^{\times}$, and put $U=S \cap x^{-1} T x$. We define the proper correspondence $X_{T S}(x)$ on $V_{S} \times V_{T}^{\sigma(x)}$ by

$$
X_{T S}(x)=J_{T U}(x) \circ{ }^{t} J_{S U}(1),
$$

where $t$ means the transpose of the correspondence. $X_{T S}(x)$ is defined over $k_{U}$. Fix $S$ and a maximal order $\mathfrak{o}$ of $B$ such that $S \subseteq B_{\infty+}^{\times} \times \prod \mathfrak{p}_{\mathfrak{p}}^{\times}$. Take and fix a finite prime $\mathfrak{p}$ of $F$ which is prime to $D(B / F) L(S)$. We fix a prime divisor $\mathfrak{R}$ of $\overline{\boldsymbol{Q}}$ which lies above $\mathfrak{p}$, and denote by $\tilde{X}$ the reduction modulo $\mathfrak{P}$ of an algebro-geometric object $X$ over $\overline{\boldsymbol{Q}}$. Finally, let $\pi$ be the $N_{F / Q}(\mathfrak{p})$-th power automorphism of the residue field of $\mathfrak{R}$, and $\Phi_{S}$ the Frobenius correspondence on $\tilde{V}_{S} \times \tilde{V}_{S}^{\pi}$.

Theorem (3.4.2) (Shimura [17] 2.23). The notation and the assumption being as above, let $x_{\mathfrak{p}}$ be an element of $\mathfrak{o}_{\mathfrak{p}}$ such that $\nu\left(x_{\mathfrak{p}}\right)$ is a prime element of $F_{p}$, and $x$ an element of $B_{A+}^{\times}$whose $\mathfrak{p}$-component is $x_{\mathfrak{p}}$ and whose other components are all equal to 1 . Suppose that $\tilde{V}_{S}$ is smooth, and $\tilde{J}_{S S}\left(\mathcal{L}(x)^{-1}\right)$ is a morphism. Then the following congruence relation holds for $\mathfrak{p}$ : 


$$
\tilde{X}_{S S}(x)={ }^{t} \Phi_{S}^{\pi^{-1}}+{ }^{t} \tilde{J}_{S S}\left(\nu(x)^{-1}\right)^{\pi^{-1}} \circ \Phi_{S}={ }^{t} \Phi_{S}^{\pi^{-1}}+\tilde{J}_{S S}(\nu(x))^{\pi} \circ \Phi_{S} .
$$

Next, for a member $S$ of $\mathscr{Z}$, let $P_{S}$ be the set of finite primes of $k_{S}$ which are prime to $D(B / F) L(S)$. We denote by $\mathfrak{r}_{S}$ the subring of $k_{S}$ consisting of those elements which are integral at all the finite primes in $P_{S}$. For $\mathfrak{q} \in P_{S}$, we denote by $\mathfrak{r}_{S q}$ the localization of $\mathfrak{r}_{S}$ at $\mathfrak{q}$.

Theorem (3.4.3) (Morita [13] Main Theorems 1 and 2, Ihara [7]). The notation being as above, we have the following two assertions.

i) There exists a projective smooth curve $W_{S}$ over $\mathfrak{r}_{S}$ whose general fibre is isomorphic to $V_{S}$.

ii) For $S, T \in \mathscr{Z}, x \in B_{A+}^{\times}$and $\mathfrak{q} \in P_{S}$ such that $x S x^{-1} \subseteq T$ and $\nu(x) \in$ $\nu(S) F_{a}^{\times} F^{\times}$, there exists an $\mathfrak{r}_{S q}-$ morphism of $W_{S} \otimes_{\mathfrak{r}_{S}} \mathfrak{r}_{S q}$ to $W_{T}^{\sigma(x)} \otimes_{\mathfrak{r}_{T}^{\sigma(x)}} \mathfrak{x}_{S q}$ whose base change to $k_{S}$ is identified with $J_{T S}(x)$ via the isomorphism in i).

This is a sharpening of Morita's original result, which says that the above assertions i) and ii) hold taking $N_{F / Q}(L(S))$ instead of $L(S)$. To deduce the refined result from Morita's, we need (1) a result of Lamprecht [9] (Korollar 5), and (2) a result of Ihara [7] (p. 308, lines 7-8). We use (1) to prove that if (3.4.3) holds for a small normal subgroup of $S$, then it also holds for $S$. On the other hand, for a small $S$, (3.4.3) follows from Morita's result combined with (2). We also have an alternative method to prove the second step above, which is closer to Morita's. However, we do not discuss them here in details. We hope to have another occasion to treat them.

Combining (3.4.2) and (3.4.3), we obtain the following

Corollary (3.4.4). Let $\mathfrak{p}$ be a finite prime of $F$ which is prime to

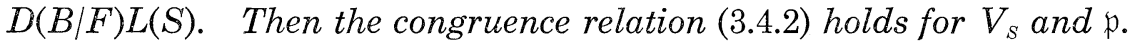

3.5. In 2.6 , we defined the operator $\mathfrak{I}^{*}(S x S)_{n}$ on $H^{1}\left(\bar{V}_{S}, \bar{F}_{n}\right)$ for $x \in A_{l} \cap$ $V(S)$ satisfying the condition in (2.6.1). Such an $x$ also satisfies the same condition for $S_{n}$, and hence we can define $\mathfrak{T}^{*}\left(S_{n} x S_{n}\right)_{n}$ on $H^{1}\left(\bar{V}_{S_{n}}, X / l^{n} X\right)$. In view of (2.6.1) and [1] XVII 6.2.3 (Var 2), these operators commute with the canonical map (3.1.1). Also for $n \leqslant m$, the operators $\mathfrak{I}^{*}\left(S_{n} x S_{n}\right)_{n}$ and $\mathfrak{I}^{*}\left(S_{m} x S_{m}\right)_{m}$ commute with the map: $H^{1}\left(\bar{V}_{S_{n}}, X / l^{n} X\right) \rightarrow H^{1}\left(\bar{V}_{S_{m}}, X / l^{n} X\right) \rightarrow$ $H^{1}\left(\bar{V}_{S_{m}}, X / l^{m} X\right)$, where the first map is the canonical map, and the second one is induced by $\tilde{i}_{m}^{n}$. Taking inductive limits, we obtain two endomorphisms of the Galois modules:

$$
\begin{aligned}
& \mathfrak{I}^{*}(S x S)=\underset{\rightarrow}{\lim } \mathfrak{I}^{*}(S x S)_{n} \in \operatorname{End}\left(\lim H^{1}\left(\bar{V}_{S}, \bar{F}_{n}\right)\right), \\
& \mathfrak{I}^{* *}(S x S)=\lim _{\rightarrow} \mathfrak{T}^{*}\left(S_{n} x S_{n}\right)_{n} \in \operatorname{End}\left(\lim _{\rightarrow} H^{1}\left(\bar{V}_{S_{n}}, X / l^{n} X\right)\right),
\end{aligned}
$$

where the first (resp. the second) inductive limit is taken relative to $\left\{i_{m}^{n}\right\}$ 
(resp. the above maps). These two operators commute with the map $\omega$ ((3.1.2)).

Now since $V_{S_{n}} \times_{V_{S}} F_{n}$ is isomorphic to the constant group scheme $V_{S_{n}} \times$ $X / l^{n} X$ over $V_{S_{n}}$, the isomorphism $H^{1}\left(\bar{V}_{S_{n}},\left(V_{S_{n}} \times_{V_{S}} F_{n}\right) \otimes_{F} \bar{F}\right) \cong H^{1}\left(\bar{V}_{S_{n}}, X / l^{n} X\right)$ is compatible with the action of $\operatorname{Gal}(\bar{F} / F)$, and hence so is $\omega$. Since the Galois group Gal $(\bar{F} / F)$ acts on the cohomologies via its action on the second factors of $\bar{V}_{S}=V_{S} \otimes_{F} \bar{F}$ and $\bar{F}_{n}=F_{n} \otimes_{F} \bar{F}$ etc., the operators $\mathfrak{I}^{*}(S x S)$ and $\mathfrak{I}^{* *}(S x S)$ are compatible with the action of $\operatorname{Gal}(\bar{F} / F)$. But we have an isomorphism:

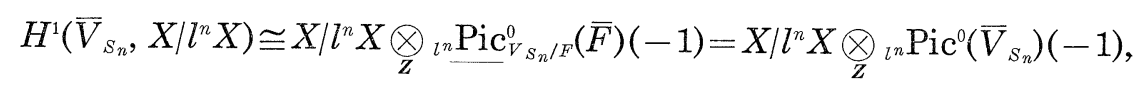

as $\operatorname{Gal}(\bar{F} / F)$-modules, where $\operatorname{Pic}_{V_{S_{n} / F}}^{0}$ denotes the identity component of the Picard scheme of the $F$-scheme $V_{S_{n}}$, and $(-1)$ denotes the Tate twist by $T_{l}\left(\boldsymbol{G}_{m}\right)^{\otimes(-1)}$. Here Gal $(\bar{F} / F)$ acts on the groups on the right hand side as $i d \otimes$ (the natural action). This gives an isomorphism of $\operatorname{Gal}(\bar{F} / F)$-modules:

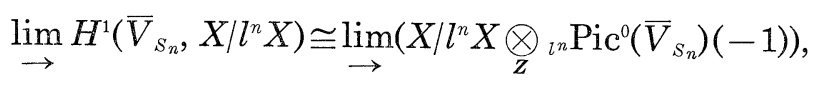

where the inductive limit in the right hand side is the natural one.

We now state consequences of the results of Morita and Shimura.

Proposition (3.5.5). The l-adic representation $\varphi_{S, \rho}$ is unramified at all the finite primes of $F$ which are prime to $l D(B / F) L(S)$.

Proof. By (3.1.3), it is enough to prove the corresponding assertion for $\lim _{\rightarrow} H^{1}\left(\bar{V}_{S_{n}}, X / l^{n} X\right)$. Since $k_{S_{n}}$ is unramified outside $l L(S)$ over $F$, our asser-

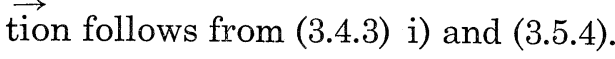

Q.E.D.

Let $\mathfrak{p}$ be a finite prime of $F$ which is prime to $l D(B / F) L(S)$. Then Shimura's congruence relation for $V_{S_{n}}$ for all $n$ implies the following assertion, in which we define the operator $B$ whose property will be studied below.

Proposition (3.5.6). For $\mathfrak{p}$ as above, take an element $x$ of $B_{A_{+}}^{\times}$as in (3.4.2). We denote by the same symbol $\mathfrak{T}^{*}(S x S)$ the endomorphism of the maximal divisible subgroup $D$ of $\lim _{\rightarrow} H^{1}\left(\bar{V}_{s}, \bar{F}_{n}\right)$ induced by the above $\mathfrak{I}^{*}(S x S)$. Then we have

$$
\mathfrak{T}^{*}(S x S)=A+B
$$

on $D$ with $A=\varphi_{S, o}\left(\sigma_{p}^{-1}\right)$, where $\sigma_{p}$ is a Frobenius element at $\mathfrak{p}$ in $\operatorname{Gal}(\bar{F} / F)$, and $B$ is an endomorphism of $D$.

Proof. Let $\xi_{p}$ be the endomorphism of $\mathrm{Pic}_{V_{S_{n} / F} / F}^{0}$ attached to the cor- 
respondence $X_{S_{n} S_{n}}(x)$; if we denote by $\delta_{p}$ the homomorphism of $\mathrm{Pic}_{V_{S_{n}} / k_{S_{n}}}^{0}$ to $\mathrm{Pic}_{V_{S_{n}}^{\sigma(x)} / k_{S_{n}}}$ defined by $X_{S_{n} S_{n}}(x)$ in the usual sense, then $\xi_{\mathrm{p}}$ is equal to $\prod_{I} \delta_{\mathfrak{p}}^{\sigma\left(y_{I}\right)}$ on $\mathrm{Pic}_{V S_{n} / F}^{0} \otimes_{F} k_{S_{n}} \cong \prod_{I} \mathrm{Pic}_{V_{S}}^{\sigma}{ }_{S}^{\left(y_{I}\right) / k_{S_{n}}}=\prod_{I}\left(\mathrm{Pic}_{V_{S_{n} / k_{S_{n}}}^{0}}\right)^{\sigma\left(y_{I}\right)}$, with $\left\{y_{I}\right\}$ as in 3.1. Now it follows from [1] XVII (6.3.18.2) that $\mathfrak{I}^{*}\left(S_{n} x S_{n}\right)_{n}$ and $i d \otimes \xi_{\mathfrak{p}} \otimes i d$ on $X / l^{n} X$ $\otimes_{Z l^{n}} \operatorname{Pic}^{0}\left(\bar{V}_{S_{n}}\right) \otimes_{Z} T_{l}\left(G_{m}\right)^{\otimes(-1)}$ commute with the isomorphism (3.5.3). In the following, we will identify the groups in (3.5.3), and hence identify $\mathfrak{I}^{*}\left(S_{n} x S_{n}\right)_{n}$ with $i d \otimes \xi_{p} \otimes i d$. Let $\mathfrak{P}$ be a prime divisor of $\overline{\boldsymbol{Q}}$ above $\mathfrak{p}$, and $\sigma_{\mathfrak{p}}$ a Frobenius element for $\mathfrak{P}$ over $\mathfrak{p}$ in $\operatorname{Gal}(\bar{F} / F)$. For $P=a \otimes t \otimes s \in X / l^{n} X \otimes_{Z{ }^{n}} \operatorname{Pic}^{0}\left(\bar{V}_{S_{n}}\right)$ $(-1)$, we put $\tilde{P}=a \otimes \tilde{t} \otimes \tilde{s}$ with the notation of 3.4. Then (3.4.2) and (3.4.4) implies that

$$
\widetilde{\mathfrak{I}} *\left(S_{n} x S_{n}\right)_{n} \tilde{P}=N_{F / Q}(\mathfrak{p})\left(a \otimes \tilde{t}^{-1} \otimes \tilde{s}\right)+\left(a \otimes \tilde{J}_{S_{n} S_{n}}\left(\nu(x)^{-1}\right)^{*} \tilde{t}^{n} \otimes \tilde{s}\right),
$$

where $*$ denotes the pullback of the divisor, and we consider $J_{S_{n} S_{n}}\left(\nu(x)^{-1}\right)$ as an automorphism of $V_{S_{n}}$ over $F$; it coincides with $\bigsqcup_{I} J_{S_{n} S_{n}}\left(\nu(x)^{-1}\right)^{\sigma\left(y_{I}\right)}$ on $\bar{V}_{S_{n}}$ $\cong \coprod_{I} V_{S_{n}}^{\sigma\left(y_{I}\right)} \otimes_{k_{S_{n}}} \bar{F}$. Since the reduction modulo $\mathfrak{P}$ is injective on $X / l^{n} X \otimes_{Z}$ ${ }_{{ }^{n}} \mathrm{Pic}^{0}\left(\bar{V}_{S_{n}}\right)(-1)$, we conclude that $\mathfrak{I}^{*}\left(S_{n} x S_{n}\right)_{n} P=A_{n} P+B_{n} P$, where $A_{n} P=$ $N_{F / Q}(\mathfrak{p})\left(a \otimes t^{\sigma_{\mathfrak{p}}^{-1}} \otimes s\right)=a \otimes t^{\sigma_{\mathfrak{p}}^{-1}} \otimes s^{\sigma_{\mathfrak{p}}^{-1}}$, and $B_{n}$ is an endomorphism of the above group. This shows that $\mathfrak{I}^{* *}(S x S)=\left(\right.$ action of $\left.\sigma_{\mathfrak{p}}^{-1}\right)+B^{\prime}$ with $B^{\prime}=\lim B_{n}$. Restricting this equality to the image of $\omega$, we obtain our assertion. $\overrightarrow{\text { Q.E.D. }}$

Proposition (3.5.7). The notation being as above, we have

$$
B A=N_{F / Q}(\mathfrak{p}) \mathfrak{T}^{*}(S \nu(x) S)
$$

on the maximal divisible subgroup of $\lim _{\rightarrow} H^{1}\left(\bar{V}_{s}, \bar{F}_{n}\right)$.

Proof. With the notation as in the proof of (3.5.6), we need to prove that $B_{n} A_{n}=N_{F / Q}(\mathfrak{p}) \mathfrak{I}^{*}\left(S_{n} \nu(x) S_{n}\right)_{n}$. Let $\eta_{\mathfrak{p}}$ be the endomorphism of $\mathrm{Pic}_{V_{S_{n} / F}}^{0}$ attached to the correspondence $X_{S_{n} S_{n}}(\nu(x))=J_{S_{n} S_{n}}(\nu(x))$. Then by the same reason as above, we have to prove that $\left(\widetilde{B_{n} A_{n} P}\right)=N_{F / Q}(\mathfrak{p})\left(i d \otimes \tilde{\eta}_{\mathfrak{p}} \otimes i d\right)(\tilde{P})$ for all $P \in X / l^{n} X \otimes_{Z l^{n}} \operatorname{Pic}^{0}\left(\bar{V}_{S_{n}}\right)(-1)$, which is obvious from (3.4.2). Q.E.D.

3.6. To compare the operator $B$ and $\varphi_{S, \rho}\left(\sigma_{p}\right)$, we need some calculations. Fix $n$, and recall the double coset decomposition $B_{A+}^{\times}=\bigcup_{I} S_{n} y_{I} B_{+}^{\times}=\bigcup_{(k, i)}$ $S_{n} s_{k} x_{i} B_{+}^{\times}$as in 3.1. We assume that $s_{1}=1$. We define the map $\lambda_{n}^{\prime}$ as the composite of the natural map: $H^{1}\left(\bar{V}_{S}, \bar{F}_{n}\right) \rightarrow \oplus_{I} H^{1}\left(V_{S_{n}}^{\sigma(y)} \otimes_{k_{S n}} \bar{F}, X / l^{n} X\right)($ cf. 2.5), and a (non-canonical) isomorphism: $\oplus_{I} H^{1}\left(V_{S_{n}} \otimes_{k_{S_{n}}} \bar{F}, X / l^{n} X\right) \rightarrow \oplus_{I} X / l^{n} X \otimes_{Z}$ ${ }_{l^{n}} \operatorname{Pic}^{0}\left(V_{S_{n}}^{\sigma\left(y_{I}\right)} \otimes_{k_{S_{n}}} \bar{F}\right)$. For simplicity, we write $\mathscr{G}_{I, n}$ for the summands of the latter group. We also define the map $\lambda_{n}$ as the composite of the above $\lambda_{n}^{\prime}$ and the projection from $\oplus_{I} \mathscr{G}_{I, n}$ to the partial sum $\oplus_{i} \mathscr{G}_{i, n}$, where $\mathscr{G}_{i, n}=X / l^{n} X$ $\otimes_{Z l^{n}} \operatorname{Pic}^{0}\left(V_{S_{n}}^{\sigma\left(x_{i}\right)} \otimes_{k_{S_{n}}} \bar{F}\right)$. Here, to define $\lambda_{n}^{\prime}$ and $\lambda_{n}$, we are fixing isomorphisms of $\mu_{l^{n}}^{\otimes(-1)}$ onto $\boldsymbol{Z} / l^{n} \boldsymbol{Z}$ which are compatible with the natural inductive limits, 
where $\mu_{l^{n}}$ denotes the group of $l^{n}$-th roots of unity in $\bar{F}$. Then $\lambda_{n}^{\prime}$ and $\lambda_{n}$ are compatible with the natural inductive limits, and by (3.1.3) and its proof, the kernels of $\lim \lambda_{n}^{\prime}$ and $\lim \lambda_{n}$ are finite. We denote by $\chi_{n}$ and $\chi_{\infty}$ the usual cyclotomic characters of $\operatorname{Gal}(\bar{F} / F)$ defined by

$$
\begin{aligned}
& \chi_{n}: \operatorname{Gal}(\bar{F} / F) \longrightarrow \operatorname{Aut}\left(\mu_{l^{n}}\right) \cong\left(Z / l^{n} Z\right)^{\times}, \\
& \chi_{\infty}: \operatorname{Gal}(\bar{F} / F) \longrightarrow \operatorname{Aut}\left(\bigcup_{n=1}^{\infty} \mu_{l^{n}}\right) \cong Z_{l}^{\times} .
\end{aligned}
$$

Lemma (3.6.2). For an element $P$ in $H^{1}\left(\bar{V}_{s}, \bar{F}_{n}\right)$ and $\sigma \in \operatorname{Gal}(\bar{F} / F)$, put $\lambda_{n}(P)=\left(t_{1}, \cdots, t_{h}\right)$ and $\lambda_{n}\left(P^{\sigma}\right)=\left(u_{1}, \cdots, u_{h}\right)$ with $t_{i}, u_{i} \in \mathscr{G}_{i, n}$. Take an element $x \in B_{A+}^{\times}$such that $\sigma(x)=\sigma$ on $k_{S_{n}}$. For each $i(1 \leqslant i \leqslant h)$, determine $j$ and $s \in S$ by the relation $x x_{i}=s x_{j} \alpha$ with $\alpha \in B_{+}^{\times}$. Then we have

$$
u_{j}=\chi_{n}(\sigma)^{-1}\left(\rho\left(s^{-1}\right) \otimes J_{S_{n} S_{n}}(s)^{\sigma\left(x_{j}\right)^{*}}\right) t_{i}^{\sigma}
$$

where $\operatorname{Gal}(\bar{F} / F)$ acts on $\mathscr{G}_{i, n}$ as $i d \otimes($ the natural action).

Proof. From the definition of $F_{n}$, we see that the image of $\lambda_{n}^{\prime}$ is invariant under the action $s \mapsto \rho\left(s^{-1}\right) \otimes\left([\tau(s)] \otimes_{F} i d_{\bar{F}}\right)^{*}$ of $S$ on $X / l^{n} X \otimes_{l^{n}} \operatorname{Pic}^{0}\left(\bar{V}_{S_{n}}\right)$ $\cong \oplus_{I} \mathscr{G}_{I, n}$. But from the definition of $\tau((2.3 .2)$, cf. also (2.3.6)), we easily see that $[\tau(s)] \otimes_{F} i d_{\bar{F}}$ is identified with $\coprod_{I} J_{S_{n} S_{n}}(s)^{\sigma\left(y_{I}\right)}$ via the identification $\bar{V}_{S_{n}} \cong$ $\amalg_{I} V_{S_{n}}^{o\left(y_{I}\right)} \otimes_{k_{S_{n}}} \bar{F}$. Our conclusion follows from this at once.

Q.E.D.

REMARK (3.6.3). By the proof, we see that the above $s$ could be any element $s$ of $S$ such that $\sigma(s)=\sigma\left(x_{j}^{-1} x x_{i}\right)$ on $k_{S_{n}}$.

Next, we recall that there is a natural isomorphism of $\oplus_{i} H^{1}\left(V_{S_{n}}^{\sigma\left(x_{i}\right)} \otimes_{k_{S_{n}}}\right.$ $\left.\bar{F}, X / l^{n} X\right)$ onto $\oplus_{i} H^{1}\left(V_{S_{i, n}} \otimes_{k_{S_{n}}} \bar{F}, X / l^{n} X\right)$ (cf. 2.5), and hence a natural isomorphism of $\oplus_{i} \mathscr{G}_{i, n}$ onto $\oplus_{i} \mathscr{H}_{i, n}$ with $\mathscr{H}_{i, n}=X / l^{n} X \otimes_{{ }^{n}} \operatorname{Pic}^{0}\left(V_{S_{i}, n} \otimes_{k_{S_{n}}} \bar{F}\right)$, the latter isomorphism being given by $\oplus_{i} \rho\left(x_{i}^{-1}\right) \otimes J_{S_{n} S_{i}, n}\left(x_{i}\right)^{*}$. Define the map $\pi_{n}$ as the composite of $\lambda_{n}$ and this isomorphism:

$$
\pi_{n}: H^{1}\left(\bar{V}_{S}, \bar{F}_{n}\right) \longrightarrow \oplus_{i} \mathscr{H}_{i, n} .
$$

Corollary (3.6.5). The notation being as above, we put $\pi_{n}(P)=\left(t_{1}^{\prime}, \cdots, t_{h}^{\prime}\right)$ and $\pi_{n}\left(P^{\sigma}\right)=\left(u_{1}^{\prime}, \cdots, u_{h}^{\prime}\right)$ with $t_{i}^{\prime}$, $u_{i}^{\prime} \in \mathscr{H}_{i, n}$. Take $x \in B_{A+}^{\times}$as above, and for each $i(1 \leqslant i \leqslant h)$, determine $j$ by the relation $x x_{i}=s^{\prime} x_{j} \alpha$ with $s^{\prime} \in S$ and $\alpha \in B_{+}^{\times}$. Then for any $s \in S$ such that $\sigma(s)=\sigma\left(x_{j}^{-1} x x_{i}\right)$ on $k_{S_{n}}$, we have

$$
u_{j}^{\prime}=\chi_{n}(\sigma)^{-1}\left(\rho\left(x_{j}^{-1} s^{-1} x_{i}\right) \otimes J_{S_{i}, n S_{j}, n}\left(x_{i}^{-1} s x_{j}\right)^{*}\right) t_{i}^{\prime \sigma} .
$$

Proof. This follows from (3.6.3) and the definition of the isomorphism of $\mathscr{G}_{i, n}$ to $\mathscr{H}_{i, n}$.

Q.E.D.

REMARK (3.6.6). In Shimura's work [19], the formula above was the 
definition of the action of the Galois group $\operatorname{Gal}(\bar{F} / F)$.

3.7. We next compute the effect of the operator $B_{n}$ on the image of $\pi_{n}$. We keep the notation of 3.4 and 3.5. Especially we fix a finite prime $\mathfrak{p}$ of $F$ which is prime to $l D(B / F) L(S)$, and a prime $\mathfrak{P}$ of $\overline{\boldsymbol{Q}}$ lying above $\mathfrak{p}$. We also fix an element $x \in B_{A+}^{\times}$as in (3.4.2) for this $\mathfrak{p}$. For an element $t_{I}$ of $\mathscr{G}_{I, n}$, we denote by $\tilde{t}_{I}$ the element of $\tilde{\mathscr{G}}_{I, n}=X / l^{n} X \otimes_{l^{n}} \widetilde{P i c}^{0}\left(V_{S_{n}}^{\sigma\left(y_{I}\right)} \otimes_{k_{S_{n}}} \bar{F}\right)$ obtained by reduction modulo $\mathfrak{R}$.

Lemma (3.7.1). For an element $P$ of $H^{1}\left(\bar{V}_{S}, \bar{F}_{n}\right)$, put $\lambda_{n}(P)=\left(t_{1}, \cdots, t_{h}\right)$. We denote by $\left(v_{1}, \cdots, v_{h}\right)$ the projection of $\lambda_{n}^{\prime} B_{n}(P)$ to $\oplus_{i} \mathscr{G}_{i, n}$. Let $\sigma_{p}$ be a Frobenius element for $\mathfrak{B}$ over $\mathfrak{p}$ in $\operatorname{Gal}(\bar{F} / F)$. Then if $x x_{i}=s x_{j} \alpha$ with $s \in S$ and $\alpha \in B_{+}^{\times}$, we have

$$
v_{j}=\left(\rho\left(s^{-1}\right) \otimes J_{S_{n} S_{n}}\left(\nu(x)^{-1} s\right)^{\sigma\left(x_{j}\right)^{*}}\right) t_{i}^{\sigma_{p}} .
$$

Proof. The assertion is trivial when the genus of $V_{S_{n}}$ is zero, and hence we assume that it is not zero. In this case, $J_{S_{n} S_{n}}\left(\nu(x)^{-1} s\right)$ has good reduction at $\mathfrak{B}$ because of the theory of the minimal models. We have to prove that $\tilde{v}_{j}=\left(\rho\left(s^{-1}\right) \otimes \tilde{J}_{S_{n} S_{n}}\left(\nu(x)^{-1} s\right)^{\sigma\left(x_{j}\right)^{*}}\right) \tilde{t}_{i}^{\pi}$. By the congruence relation (3.4.2) for $S_{n}$, we see that $B_{n}$ maps $\tilde{t}_{i}$ to $\left(i d \otimes\left(\tilde{J}_{S_{n} S_{n}}\left(\nu(x)^{-1}\right)^{\sigma\left(x_{i}\right)}\right)^{\pi^{-1 *}}\right) \tilde{t}_{i}^{\pi} \in \mathscr{G}_{J, n}$ with $\sigma\left(y_{J}\right)=\sigma\left(s x_{j}\right)$ on $k_{S_{n}}$. Hence composing this with the action $\left(\rho\left(s^{-1}\right) \otimes \tilde{J}_{S_{n} S_{n}}(s)^{\sigma\left(x_{j}\right)^{*}}\right)$ by $s$, which leaves the image of $\lambda_{n}^{\prime}$ invariant, we obtain our assertion. Q.E.D.

Transposing this action to $\oplus_{i} \mathscr{H}_{i, n}$, we obtain the following

Corollary (3.7.2). The notation being as above, put $\pi_{n}(P)=\left(t_{1}^{\prime}, \cdots, t_{h}^{\prime}\right)$. We denote by $\left(v_{1}^{\prime}, \cdots, v_{h}^{\prime}\right)$ the image of the above $\left(v_{1}, \cdots, v_{h}\right)$ to $\oplus_{i} \mathscr{H}_{i, n}$ under het natural isomorphism of $\oplus_{i} \mathscr{G}_{i, n}$ to $\oplus_{i} \mathscr{H}_{i, n}$. Then we have

$$
v_{j}^{\prime}=\left(\rho\left(x_{j}^{-1} s^{-1} x_{i}\right) \otimes J_{S_{i}, n S_{j}, n}\left(x_{i}^{-1} \nu(x)^{-1} s x_{j}\right)^{*}\right) t_{i}^{\prime \sigma_{\mathfrak{p}}} .
$$

Now from the relation $x x_{i}=s x_{j} \alpha$, we see that

$$
x_{i}^{-1} \nu(x)^{-1} s x_{j}=\nu\left(x_{i}^{-1} x x_{i}\right)^{-1} x_{i}^{-1} s x_{j}=x_{i}^{\prime} s^{-\iota} x_{j}^{-\iota} \nu(\alpha)^{-1} .
$$

For an idele $y \in F_{A}^{\times}$, let $i l(y)$ denote the ideal of $F$ corresponding to $y$. Put $A_{i}=N_{F^{\prime} / Q}\left(i l\left(\nu\left(x_{i}\right)\right)\right)$. Then we have $\chi_{n}(\sigma(x))=\chi_{n}\left(\left[N_{F^{\prime} / Q}\left(\nu\left(x_{i}^{-1} x x_{i}\right)\right)^{-1}, \boldsymbol{Q}\right]\right)=$ $\left(A_{i} A_{j}^{-1} N_{F^{\prime} / Q}\left(\nu\left(x_{i}^{-1} s x_{j}\right)\right)\right)_{l} \bmod l^{n}$, where [, $\left.\boldsymbol{Q}\right]$ denotes the Artin symbol for $\boldsymbol{Q}$, and $y_{l}$ denotes the $l$-component of an idele $y \in \boldsymbol{Q}_{A}^{\times}$. By (1.1.2), we have

$$
\chi_{n}(\sigma(x))^{-w}=\left(A_{i}^{-w} A_{j}^{w} \rho\left(\nu\left(x_{j}^{-1} s^{-1} x_{i}\right)\right)\right)_{l} \bmod l^{n},
$$

and hence

$$
\left(\rho\left(x_{j}^{-1} s^{-1} x_{i}\right)\right)_{l} \bmod l^{n}=\chi_{n}(\sigma(x))^{-w}\left(A_{i}^{w} A_{j}^{-w} \rho\left(x_{j}^{\iota} s^{\iota} x_{i}^{-\iota}\right)\right)_{l} \bmod l^{n} .
$$


We have thus obtained the following

Corollary (3.7.3). The notation being as in (3.7.2), we have

$$
v_{j}^{\prime}=\chi_{n}\left(\sigma_{p}\right)^{w+1}\left(\chi_{n}\left(\sigma_{p}\right)^{-1} A_{i}^{w} A_{j}^{-w} \rho\left(x_{j}^{\iota} s^{\imath} x_{i}^{-\imath}\right) \otimes J_{S_{i}, n S_{j}, n}\left(x_{i}^{\imath} s^{-\imath} x_{j}^{-\imath}\right)^{*}\right) t_{i}^{\prime \sigma_{p}} .
$$

3.8. We assume here that $S$ is stable under the canonical involution $\iota ;$ $S=S^{\iota}$. For $\left\{x_{i}\right\}$ as in (1.4.2), put $y_{i}=x_{i}^{-\bullet}$ for each $i(1 \leqslant i \leqslant h)$. For each $i$, we define $\bar{i}, s_{i} \in S$ and $\alpha_{i} \in B_{+}^{\times}$by the relation $y_{i}=s_{i} x_{i} \alpha_{i}$. It is obvious that $i \mapsto \bar{i}$ is a permutation of $\{1, \cdots, h\}$ of order two. Since $\Gamma_{S_{y_{i}}}=\Gamma_{S_{i}}=\Gamma_{S_{x_{i}}}=\alpha_{i}^{-1} \Gamma_{S_{\bar{i}}} \alpha_{i}$, with $S_{x_{i}}=x_{i}^{-1} S x_{i}$ etc., we see that $f \mapsto A_{i}^{-w} f \mid\left[\alpha_{i}\right]$ gives an isomorphism of $\widetilde{S}_{m_{k}+2}\left(\Gamma_{S_{i}}, \Psi_{k}\right)$ onto $\widetilde{S}_{m_{k}+2}\left(\Gamma_{S_{i}}, \Psi_{k}\right)$ for each $k(1 \leqslant k \leqslant r)$. It is easy to see that this isomorphism does not depend on the choice of $\alpha_{i}$. Taking the direct sum of these isomorphisms over all $i$ and $k$, we obtain an automorphism $\mathfrak{Y}$ of $\widetilde{S}(S, \rho)$ :

$$
\mathfrak{V}: \Im(S, \rho) \stackrel{\sim}{\longrightarrow}(S, \rho) .
$$

Since $\alpha_{i} \in A_{l}$ (cf. 2.1), we have the following cartesian diagram:

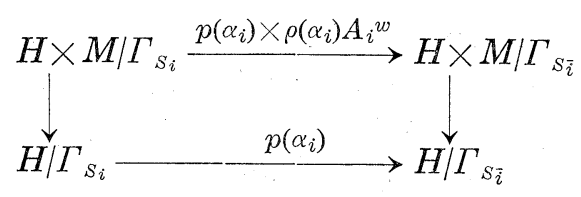

for $M=\boldsymbol{R}^{m}$ or $X / l^{n} X$. As in (2.2.4), we see that the direct sum of the canonical maps: $\left.H^{1}\left(H / \Gamma_{S_{\bar{i}}}, H \times \boldsymbol{R}^{m} / \Gamma_{S_{\bar{i}}}\right) \rightarrow H^{1}\left(H / \Gamma_{S_{i}}, p\left(\alpha_{i}\right) *\left(H \times \boldsymbol{R}^{m}\right) / \Gamma_{S_{\bar{i}}}\right)\right) \cong$ $H^{1}\left(H / \Gamma_{S_{i}}, H \times \boldsymbol{R}^{m} / \Gamma_{S_{i}}\right)$ corresponds to $\mathfrak{V}$ via the isomorphisms in (2.2.4) and (2.1.4). Similarly from the cartesian diagram:

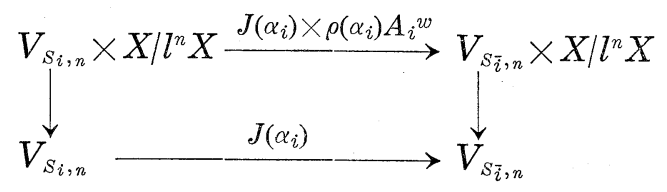

we can form the quotient by the equivariant actions of $S_{i} /\left(S_{i} \cap F^{\times}\right) S_{i, n}$ and $S_{\bar{i}} /\left(S_{\bar{i}} \cap F^{\times}\right) S_{\bar{i}, n}=\alpha_{i}\left(S_{i} /\left(S_{i} \cap F^{\times}\right) S_{i, n}\right) \alpha_{i}^{-1}$ as before, and obtain the following cartesian diagram:

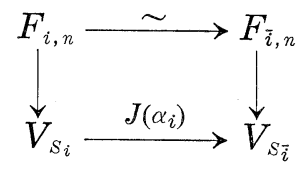

We then obtain an isomorphism of $H^{1}\left(V_{S_{\bar{i}}} \otimes_{k_{S}} \bar{F}, F_{\bar{i}, n} \otimes_{k_{S}} \bar{F}\right)$ onto $H^{1}\left(V_{S_{i}} \otimes_{k_{S}} \bar{F}\right.$, $\left.F_{i, n} \otimes_{k_{S}} \bar{F}\right)$ by the canonical map. Identifying $H^{1}\left(\bar{V}_{S}, \bar{F}_{n}\right)$ with $\oplus_{i} H^{1}\left(V_{S_{i}} \otimes_{k_{S}} \bar{F}\right.$, 
$\left.F_{i, n} \otimes_{k_{S}} \bar{F}\right)$ by the isomorphism (2.5.3), we denote by $\mathfrak{Y}_{l, n}$ the automorphism of $H^{1}\left(\bar{V}_{s}, \bar{F}_{n}\right)$ corresponding to the direct sum of the above defined isomorphisms. We put $\mathfrak{Y}_{l}=\lim _{\rightarrow} \mathfrak{Y}_{l, n} \in \operatorname{Aut}\left(\lim _{\rightarrow} H^{1}\left(\bar{V}_{s}, \bar{F}_{n}\right)\right)$.

Theorem (3.8.5). Suppose that $S$ is stable under the canonical involution (and satisfies (2.4.1)). Let $\mathfrak{p}$ be a finite prime of $F$ which is prime to $l D(B / F) L(S)$, and $\sigma_{\mathfrak{p}}$ a Frobenius element at $\mathfrak{p}$ in $\mathrm{Gal}(\bar{F} / F)$. Then we have

$$
\mathfrak{I}^{*}(S x S)=\varphi_{S, \rho}\left(\sigma_{\mathfrak{p}}^{-1}\right)+\chi_{\infty}\left(\sigma_{\mathfrak{p}}\right)^{w+1} \mathfrak{Y}_{l} \varphi_{S, \rho}\left(\sigma_{\mathfrak{p}}\right) \mathfrak{Y}_{l}^{-1},
$$

on the maximal divisible subgroup of $\lim H^{1}\left(\bar{V}_{S}, \bar{F}_{n}\right)$, where $x$ is as in (3.4.2) for $\mathfrak{p}$.

Proof. We have to prove that the operator $B$ in (3.5.6) coincides with the second factor of the right hand side of the above equality. By (3.1.3), it is sufficient to prove the corresponding assertion for the image of $\lim \pi_{n}$ : $\lim H^{1}\left(\bar{V}_{s}, \bar{F}_{n}\right) \rightarrow \oplus_{i} \lim \mathscr{H}_{i, n}$. But by the definition of $\mathfrak{V}_{l}$, it commutes with $\vec{\oplus} \lim \left(A_{i}^{-w} \rho\left(\alpha_{i}^{-1}\right) \otimes \overrightarrow{J_{S_{i}, n} S_{i}, n}\left(\alpha_{i}\right)^{*}\right)$ on $\oplus_{i} \lim \mathscr{H}_{i, n}$ via $\lim \pi_{n}$. In view of the explicit descriptions (3.6.5) and (3.7.3), if $x x_{i}=s x_{j} \alpha \overrightarrow{\text { and }} x^{-i} x_{\bar{i}}=\bar{s} x_{\bar{j}} \bar{\alpha}$ as before,

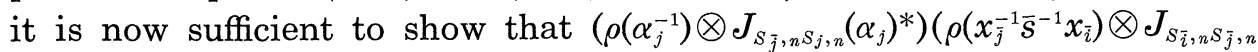
$\left.\left.\left(x_{\bar{i}}^{-1} \bar{s} x_{\bar{j}}\right)^{*}\right)\left(\rho\left(\alpha_{i}\right) \otimes J_{S_{i}, n s_{\bar{i}, n}}\left(\alpha_{i}^{-1}\right)^{*} t_{i}^{\prime}\right)^{\sigma_{p}}=\rho\left(x_{j}^{\imath} s^{\imath} x_{i}^{-\imath}\right) \otimes J_{S_{i}, n S_{j}, n}\left(x_{i}^{t} s^{-\imath} x_{j}^{-\imath}\right)^{*}\right) t_{i}^{\sigma_{p}}$, for all $i, n$ and $t_{i}^{\prime} \in \mathscr{H}_{i, n}$. Put $s^{\prime}=s_{i} \bar{s}_{j}^{-1}$. Then the left hand side of the above is equal to $\left(\rho\left(x_{j}^{t} s^{\prime-1} x_{i}^{-t}\right) \otimes J_{S_{i, n} s_{j, n}}\left(x_{i}^{t} s^{\prime} x_{j}^{-t}\right)^{*}\right) t_{i}^{\sigma_{\sigma_{p}}}$. On the other hand, $\sigma(\bar{s})=$ $\sigma\left(s_{i}^{-1} s^{-t} s_{j}\right)$ on $F_{a b}$. By (3.6.5), changing $\bar{s}$ by $s_{i}^{-1} s^{-t} s_{j}$ in the left hand side of the above, we obtain our conclusion.

Q.E.D.

\section{$\S 4$. The $l$-adic representation attached to $\mathbb{S}(S, \rho)$}

4.1. In previous sections, starting from a representation $\rho$ satisfying (1.1.1), (1.1.2) and (2.1.6), we constructed and studied the representation $\varphi_{S, \rho}$ of $\operatorname{Gal}(\bar{F} / F)$. In order to ensure that $\varphi_{S, \rho}$ has a good property, we now impose one more condition on $\rho$ :

(4.1.1) There exists a non-degenerate bilinear form (, ): $\boldsymbol{Q}^{m} \times \boldsymbol{Q}^{m} \rightarrow \boldsymbol{Q}$ on $\boldsymbol{Q}^{m}$ which satisfies $(\rho(\alpha) x, \rho(\alpha) y)=N_{F / Q}(\nu(\alpha))^{w}(x, y)$ for all $x, y \in \boldsymbol{Q}^{m}$ and $\alpha \in B^{\times}$, where $w$ is an integer in (1.1.2).

Let $X$ be a lattice in $\boldsymbol{Q}^{m}$ as in (2.1.6) (for a maximal order $\left.\mathfrak{0}\right)$. Then, multiplying (, ) by an integer if necessary, we may suppose that the following condition is also satisfied:

$$
(X, X) \subseteq Z
$$


Put $\hat{\boldsymbol{Z}}=\boldsymbol{R} \times \prod_{p} \boldsymbol{Z}_{p}$, where the product in the right hand side ranges over all the rational primes, and $\hat{X}=X \otimes_{Z} \hat{Z}$. Then (,) induces a bilinear pairing $():, \hat{X} \times \hat{X} \rightarrow \hat{Z}$. For any $s \in\left(\mathfrak{o} \otimes_{Z} \hat{Z}\right)$ and $x, y \in \hat{X}$, we have

$$
(\rho(s) x, \rho(s) y)=N_{F / Q}(\nu(s))^{w}(x, y) .
$$

Now let $S$ be a member of $\mathscr{Z}$ which is contained in $\left(\mathfrak{0} \otimes_{Z} \hat{Z}\right)^{\times}$. Then $\hat{X} / l^{n} \hat{X} \cong X / l^{n} X$ is, as before, an $S$-module by $\rho$, and (, ) induces a pairing

$$
(,)_{n}: X / l^{n} X \times X / l^{n} X \longrightarrow Z / l^{n} Z
$$

which satisfies $(\rho(s) x, \rho(s) y)_{n}=N_{F / Q}(\nu(s))^{w}(x, y)_{n}$, where the latter product means the product of the $l$-component of $N_{F / Q}(\nu(s))^{w}$ and $(x, y)_{n}$ in the obvious sense. We now let $S$ act on $Z / l^{n} Z$ by $s \mapsto \eta(s)=\left(\right.$ multiplication by $\left.N_{F / Q}(\nu(s))^{w}\right)$, and hence consier $(,)_{n}$ as a pairing of $S$-modules which satisfies the following compatibility condition:

$$
(\rho(s) x, \rho(s) y)_{n}=\eta(s)(x, y)_{n} .
$$

Suppose, as before, that $S$ satisfies the condition (2.4.1). From (, $)_{n}$, we obtain a pairing of constant group schemes over $V_{S_{n}}$ :

$$
\left(X / l^{n} X \times V_{S_{n}}\right) \times\left(X / l^{n} X \times V_{S_{n}}\right) \longrightarrow Z / l^{n} \boldsymbol{Z} \times V_{S_{n}} .
$$

If we let $S /\left(S \cap F^{\times}\right) S_{n}$ act on $X / l^{n} X \times V_{S_{n}}$ (resp. $Z / l^{n} Z \times V_{S_{n}}$ ) by $\rho() \times[\tau()]$ (resp. $\eta() \times[\tau()])$, the above pairing is compatible with these actions. Taking the quotient by these actions, we obtain a pairing of finite étale group schemes over $V_{S}$, which we denote by $\langle,\rangle_{n}$ :

$$
\langle,\rangle_{n}: F_{n} \times F_{n} \longrightarrow\left(S /\left(S \cap F^{\times}\right) S_{n}\right) \backslash\left(Z / l^{n} Z \times V_{S_{n}}\right) .
$$

It is obvious that the base change of $\langle,\rangle_{n}$ to $V_{S_{n}}$ is identified with the pairing (4.1.6).

Lemma (4.1.8). $\quad\left(S /\left(S \cap F^{\times}\right) S_{n}\right) \backslash\left(\boldsymbol{Z} / l^{n} \boldsymbol{Z} \times V_{S_{n}}\right)$ is isomorphic to $\boldsymbol{\mu}_{l^{n}}{ }^{\otimes(-w)}$ as

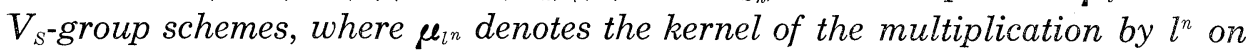
the multiplicative group $\boldsymbol{G}_{m}$.

Proof. Since $k_{S_{n}}$ corresponds to $F^{\times} \nu\left(S_{n}\right)$ by class field theory, it contains all the $l^{n}$-th roots of unity in $\overline{\boldsymbol{Q}}$, and hence there is an isomorphism $f$ of $\boldsymbol{\mu}_{l^{n}}^{\otimes(-w)}$ onto the constant group scheme $\boldsymbol{Z} / l^{n} \boldsymbol{Z}$ over $k_{S_{n}}$. Since $\tau(s)=\sigma(s)$ on $F_{a b}((2.3 .3))$, it is enough to show that the following diagram commutes for all $s \in S$ : 


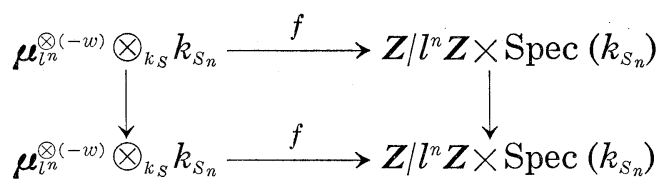

where the left (resp. the right) vertical morphism is $i d \times \operatorname{Spec}(\sigma(s)$ ) (resp. $\eta(s) \times \operatorname{Spec}(\sigma(s)))$. But $\sigma(s)$ acts on $\mu_{l_{n}}^{\otimes(-w)}(\bar{F})$ as the multiplication by $N_{F / Q}(\nu(s))^{-w}=\eta(s)^{-1}$, and our assertion follows from this.

Q.E.D.

4.2. We now consider $\langle,\rangle_{n}$ as a pairing with values in $\boldsymbol{\mu}_{l^{n}}^{\otimes(-w)}$. Denote by $i_{m}^{\prime n}$ (resp. $\dot{j}_{n}^{\prime m}$ ) the natural inclusion of $\boldsymbol{\mu}_{l^{n}}^{\otimes(-w)}$ into $\boldsymbol{\mu}_{l^{m}}^{\otimes(-w)}$ (resp. the morphism of $\boldsymbol{\mu}_{l^{m}}^{\otimes(-w)}$ to $\boldsymbol{\mu}_{l^{n}}^{\otimes(-w)}$ induced by the multiplication by $\left.l^{m-n}\right)$ for $n \leqslant m$. We obviously have

$$
\begin{aligned}
& \left\langle i_{m}^{n}, i_{m}^{n}\right\rangle_{m}=l^{m-n} i_{m}^{\prime n}\langle,\rangle_{n}, \\
& \left\langle j_{n}^{m}, j_{n}^{m}\right\rangle_{n}=\dot{j}_{n}^{\prime m}\langle,\rangle_{m},
\end{aligned}
$$

for all $n \leqslant m$. Using $\langle,\rangle_{n}$, we obtain the cup product pairing for the cohomology groups:

$$
H^{1}\left(\bar{V}_{S}, \bar{F}_{n}\right) \times H^{1}\left(\bar{V}_{S}, \bar{F}_{n}\right) \stackrel{\cup}{\longrightarrow} H^{2}\left(\bar{V}_{s}, \boldsymbol{\mu}_{l^{n}}^{\otimes(-w)}\right) .
$$

But we have the canonical isomorphisms $H^{2}\left(\bar{V}_{S}, \boldsymbol{\mu}_{l^{n}}^{\otimes(-w)}\right) \cong H^{2}\left(\bar{V}_{S}, \boldsymbol{\mu}_{l^{n}}\right) \otimes$

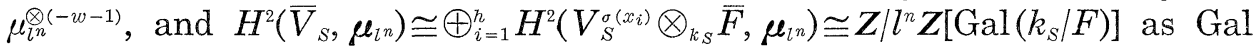
$(\bar{F} / F)$-modules. Composing (4.2.3) with

$$
H^{2}\left(\bar{V}_{S}, \boldsymbol{\mu}_{l^{n}}^{\otimes(-w)}\right) \cong \stackrel{h}{\oplus} \mu_{l^{n}}^{\otimes(-w-1)} \stackrel{\text { sum }}{\longrightarrow} \mu_{l^{n}}^{\otimes(-w-1)},
$$

where sum means the multiplication of the group $\mu_{l^{n}}^{\otimes(-w-1)}$, we obtain a pairing:

$$
H^{1}\left(\bar{V}_{S}, \bar{F}_{n}\right) \times H^{1}\left(\bar{V}_{S}, \bar{F}_{n}\right) \longrightarrow \mu_{l^{(n-w-1)}}^{\otimes} .
$$

Taking projective limits relative to $\left\{j_{n}^{m}\right\}$ and $\left\{j_{n}^{\prime m}\right\}$, and tensoring $\boldsymbol{Q}_{l}$, we obtain finally a pairing of $\boldsymbol{Q}_{l}$-vector spaces:

$$
\left(\lim _{\leftarrow} H^{1}\left(\bar{V}_{S}, \bar{F}_{n}\right)\right) \underset{Z_{l}}{\otimes} \boldsymbol{Q}_{l} \times\left(\lim _{\leftarrow} H^{1}\left(\bar{V}_{S}, \bar{F}_{n}\right) \underset{Z_{l}}{\bigotimes_{l}} \boldsymbol{Q}_{l}\right) \longrightarrow \boldsymbol{Q}_{l}(-w-1)
$$

where $\boldsymbol{Q}_{l}(-w-1)$ is as before, the Tate twist of $\boldsymbol{Q}_{l}$. This pairing is of course compatible with the action of $\operatorname{Gal}(\bar{F} / F)$.

Proposition (4.2.7). The pairing (4.2.6) is non-degenerate.

Proof. This is a consequence of the Poincaré duality ([1] XVIII 3.2.5 and Deligne [4] 2.8).

Q.E.D. 
4.3. We can now state and prove our main result, in which we now drop the assumption (2.4.1) for $S$.

THEOREM (4.3.1). Let $\rho$ be a representation of $B^{\times}$satisfying the conditions (1.1.1), (1.1.2), (2.1.6) and (4.1.1). Let $S$ be a member of $\mathscr{Z}$ which is stable under the canonical involution of $B_{A}^{\times}$. Then, for each rational prime $l$, there exists a continuous representation $\psi_{S, \rho, l}=\psi_{S, \rho}$ of the Galois group;

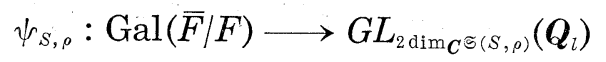

which has the following properties:

(4.3.1.1) $\psi_{S, \rho, l}$ is unramified at all the finite primes of $F$ which are prime to $l D(B / F) L(S)$.

(4.3.1.2) For a finite prime pof $F$ which is prime to $l D(B / F) L(S)$, let $\sigma_{\mathfrak{p}}$ be a Frobenius element at $\mathfrak{p}$ in $\operatorname{Gal}(\bar{F} / F)$. Then we have

$$
\operatorname{det}\left(1-\psi_{S, \rho}\left(\sigma_{p}\right) T\right)=H_{p}(T ; \Im(S, \rho))
$$

(cf. (1.5.7)).

4.4. We first prove the theorem above under an additional assumption (2.4.1) for $S$, in which case we can use our results in previous sections. We consider here the space $\left(\lim _{\leftarrow} H^{1}\left(\bar{V}_{S}, \bar{F}_{n}\right)\right) \otimes_{Z_{l}} \boldsymbol{Q}_{l}$, which we write $W_{S, \rho, l}=W_{S, \rho}$ for simplicity. We consider $\varphi_{S, \rho}$ as a representation on this space (cf. (2.5.8)). Denote by $\mathfrak{I}_{l}(S x S)$ the operator induced by $\lim _{\leftarrow} \mathfrak{T}^{*}(S x S)_{n}$ (cf. (2.6.6)) on $W_{S, \rho}$ for an element $x \in A_{l} \cap V(S)$ satisfying the condition in (2.6.1). By (2.5.4), (2.5.5) and the argument after it, we have an isomorphism:

$$
W_{S, \rho} \underset{Q_{l}}{\otimes} C \cong \subseteq(S, \rho) \underset{R}{\otimes} C
$$

where we fix an embedding of $\boldsymbol{Q}_{l}$ into $C$. By (2.1.5), (2.2.5), and (2.6.7), the operators $\mathfrak{I}_{l}(S x S) \otimes i d_{C}$ and $\mathfrak{I}(S x S) \otimes i d_{C}$ commute with the isomorphism (4.4.1). Fix a finite prime $\mathfrak{p}$ of $F$ which is prime to $l D(B / F) L(S)$, and an element $x \in B_{A+}^{\times}$as in (3.4.2) for this $\mathfrak{p}$. Then we have

$$
\begin{aligned}
& \operatorname{det}\left(1-\mathfrak{I}_{l}(S x S) T+N_{F / Q}(\mathfrak{p}) \mathfrak{I}_{l}(S \nu(x) S) T^{2} \mid W_{S, \rho}\right) \\
& \quad=\operatorname{det}\left(1-\mathfrak{I}(\mathfrak{p}) T+N_{F / Q}(\mathfrak{p}) \mathfrak{I}(\mathfrak{p}, \mathfrak{p}) T^{2} \mid \Im(S, \rho) \underset{\boldsymbol{R}}{\otimes} C\right) .
\end{aligned}
$$

By (1.7.5), the right hand side of (4.4.2) is equal to $H_{p}(T ; \Im(S, \rho))^{2}$, since $S=$ $S^{\prime}$.

We define the representation $\psi_{S, \rho, l}=\psi_{S, \rho}$ by

$$
\psi_{S, \rho}(\sigma)=\varphi_{S, \rho}\left(\sigma^{-1}\right)
$$


for all $\sigma \in \operatorname{Gal}(\bar{F} / F)$. Then the assertion (4.3.1.1) is nothing but (3.5.5). Let $\mathfrak{Y}_{l}^{\prime}$ be the automorphism of $W_{S, \rho}$ induced by $\lim \mathfrak{Y}_{l, n}$ (cf. 3.8). Then our congruence relation (3.8.5) implies

$$
\mathfrak{I}_{l}(S x S)=\psi_{S, \rho}\left(\sigma_{\mathfrak{p}}\right)+\chi_{\infty}\left(\sigma_{\mathfrak{p}}\right)^{w+1} \mathfrak{Y}_{l}^{\prime} \psi_{S, \rho}\left(\sigma_{\mathfrak{p}}^{-1}\right) \mathfrak{Y}_{l}^{\prime-1}
$$

on $W_{S, \rho}$. This together with (3.5.7) implies

$$
H_{p}(T ; \Im(S, \rho))^{2}=\operatorname{det}\left(1-\psi_{S, \rho}\left(\sigma_{p}\right) T\right) \operatorname{det}\left(1-\chi_{\infty}\left(\sigma_{p}\right)^{w+1} \psi_{S, \rho}\left(\sigma_{p}^{-1}\right) T\right) .
$$

But the adjoint of $\psi_{S, \rho}\left(\sigma_{p}\right)$ with respect to the pairing (4.2.6) is $\chi_{\infty}\left(\sigma_{p}\right)^{w+1} \psi_{S, \rho}\left(\sigma_{p}^{-1}\right)$. By (4.2.7), we conclude that

$$
\operatorname{det}\left(1-\psi_{S, \rho}\left(\sigma_{\mathfrak{p}}\right) T\right)^{2}=H_{p}(T ; \Im(S, \rho))^{2} .
$$

Taking the square roots, we obtain (4.3.1.2).

4.5. We next consider the general case. For $S \subseteq B_{\infty+}^{\times} \times \prod \mathfrak{o}_{p}^{\times}$as in (4.3.1), fix a prime number $q$ which is prime to $l L(S)$. Take a positive integer $n$ so large that $T=S \cap\left(B_{\infty+}^{\times} \times \prod_{q \mid q} \mathfrak{D}_{q}^{\times} \times \prod_{q \mid q}\left(1+q^{n} \mathfrak{o}_{q}\right)\right)$ satisfies (2.4.1). The group $S /\left(S \cap F^{\times}\right) T$ acts on $W_{T, \rho}$ by $s \mapsto \mathfrak{I}_{l}(T s T)$. Put

$$
W_{S, \rho}=W_{T, \rho}^{\left(S /\left(S \cap F^{\times}\right) T^{T}\right)} \text {. }
$$

By (4.4.1) and the argument after it, we see that $W_{S, \rho} \otimes_{Q_{l}} C \cong \Im(S, \rho) \otimes_{R} C$ by (1.6.3). Since $\mathfrak{I}_{l}(T s T)$ and $\psi_{T, \rho}(\sigma)$ commute for all $s \in S$ and $\sigma \in \operatorname{Gal}(\bar{F} / F)$, $W_{S, \rho}$ is a $\operatorname{Gal}(\bar{F} / F)$-submodule of $W_{T, \rho}$. We denote by $\psi_{S, \rho}$ the representation of $\operatorname{Gal}(\bar{F} / F)$ on this space obtained by restricting $\psi_{T, \rho}$;

$$
\psi_{S, \rho}: \operatorname{Gal}(\bar{F} / F) \longrightarrow \operatorname{Aut}_{Q_{l}}\left(W_{S, \rho}\right) \cong G L_{2 \operatorname{dim}_{C^{\Phi}(S, \rho)}}\left(\boldsymbol{Q}_{l}\right) .
$$

LEMMA (4.5.3). $\quad \psi_{s, \rho}$ is unramified at all the finite primes of $F$ which are prime to $l D(B / F) L(S)$.

Proof. In general, let $T_{1}$ be a member of $\mathscr{Z}$ which satisfies (2.4.1), and let $T_{2}$ be a normal subgroup of $T_{1}$ which is also a member of $\mathscr{Z}$. Then $V_{T_{2}}$ is an étale Galois covering of $V_{T_{1}}$ with group $T_{1} /\left(T_{1} \cap F^{\times}\right) T_{2}$. By the definition of $F_{T_{1}, n}$, there is a canonical isomorphism of $J_{T_{1} T_{2}}(1)^{*} F_{T_{1}, n}=F_{T_{1}, n} \times_{V_{T_{1}}}$ $V_{T_{2}}$ onto $F_{T_{2}, n}$, which gives an isomorphism of inductive (resp. projective) system of étale sheaves on $V_{T_{2}}$. This induces the canonical map $i: W_{T_{1}, \rho} \rightarrow$ $W_{T_{2}, \rho}$ which commutes with the action of $\operatorname{Gal}(\bar{F} / F)$. It is easy to see that $i \otimes_{Q} i d_{C}$ corresponds, via the isomorphism (4.4.1), to the natural injection of $\Im\left(T_{1}, \rho\right) \otimes_{R} C$ into $\subseteq\left(T_{2}, \rho\right) \otimes_{R} C$. From (1.6.3), we conclude that $i$ induces an isomorphism of $W_{T_{1}, \rho}$ onto $W_{T_{2}, \rho}^{\left(T_{1} /\left(T_{1} \cap F^{\times}\right) T_{2}\right)}$.

Now for $S$, take $q$ and $T$ as above. Let $q^{\prime}$ be a prime number which is 
prime to $q l L(S)$, and take a positive integer $n^{\prime}$ so large that $T^{\prime}=S \cap\left(B_{\infty+}^{\times} \times\right.$ $\left.\prod_{\mathfrak{q} \times q^{\prime} \mathfrak{D}_{\mathfrak{q}}^{\times}} \times \prod_{\mathfrak{q} \mid q^{\prime}}\left(1+q^{\prime n^{\prime}} \mathfrak{D}_{q}\right)\right)$ satisfies (2.4.1). Applying the above argument for $T_{1}=T$ or $T^{\prime}$ and $T_{2}=T \cap T^{\prime}$, we see that $W_{S, \rho}=W_{T, \rho}^{\left(S /\left(S \cap F^{\times}\right) T\right)} \cong W_{T^{\prime}, \rho}^{\left(S /\left(S \cap F^{\times}\right) T^{\prime}\right)}$ as $\operatorname{Gal}(\bar{F} / F)$-modules. Our assertion then follows from (3.5.5).

Q.E.D.

In view of (1.6.2) and the proof in 4.4, the proof of (4.3.1.2) for $\psi_{s, \rho}$ is reduced to the following two lemmas.

LEMMA (4.5.4). The subspace $W_{S, \rho}$ of $W_{T, \rho}$ is stable under the operator $\mathfrak{Y}_{l}^{\prime}$ on $W_{T, \rho}$.

Proof. By the definition of $\mathfrak{Y}_{l, n}$ (cf. 3.8), and the isomorphism (4.4.1), it is sufficient to show that $\mathfrak{S}(S, \rho)$ is stable under the operator $\mathfrak{V}$ on $\mathfrak{S}(T, \rho)$. But we have a disjoint decomposition $B_{A+}^{\times}=\bigcup_{k, i} T s_{k} x_{i} B_{+}^{\times}$, where $s_{k} \in S$ and $\left\{x_{i}\right\}$ is as in (1.4.2). Hence $\left(s_{k} x_{i}\right)^{-\imath}=t\left(s_{k^{\prime}}, x_{\bar{i}}\right) \alpha$ with $t \in T$ and $\alpha \in B_{+}^{\times}$implies $x_{i}^{-\iota}$ $=\left(s_{k}^{\prime} t s_{k^{\prime}}\right) x_{i} \alpha$. Since $S=S^{\prime}$, we obtain our assertion immediately.

Q.E.D.

LEMMA (4.5.5). The restriction of the pairing (4.2.6) to $W_{S, \rho}$ is non-degenerate.

Proof. Let us denote by $(,)_{l}$ the pairing (4.2.6). We first claim that $\left(\mathfrak{I}_{l}(T s T) x, \mathfrak{I}_{l}(T s T) y\right)_{l}=(x, y)_{l}$ holds for all $x, y \in W_{T, \rho}$ and $s \in S$. In fact, for $s \in S$, consider the following cartesian diagram:

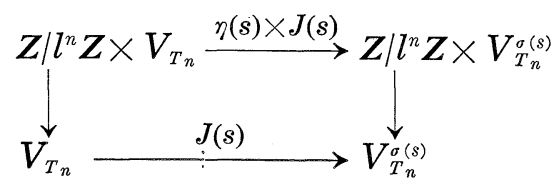

As in (2.6.2), we have $(\eta(s) \times J(s))(\eta(t) \times[\tau(t)])=\left(\eta\left(s t s^{-1}\right) \times\left[\tau\left(s t s^{-1}\right)\right]^{\sigma(s)}\right)(\eta(s) \times$ $J(s)$ ) for all $t \in T$. Hence taking the quotient by the action of $T /\left(T \cap F^{\times}\right) T_{n}$, we have the following cartesian diagram:

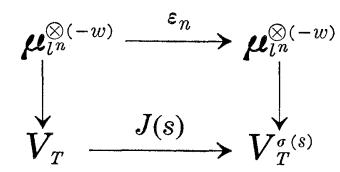

Identifying the upper left $\boldsymbol{\mu}_{l^{n}}^{\otimes(-w)}$ with $J(s)^{*} \boldsymbol{\mu}_{l^{n}}^{\otimes(-w)}$ by $\varepsilon_{n}$, we have the trace map:

$$
t(s)_{n}: H^{2}\left(\bar{V}_{T}, \boldsymbol{\mu}_{l^{n}}^{\otimes(-w)}\right) \longrightarrow H^{2}\left(\overline{V_{T}^{\sigma(s)}}, \boldsymbol{\mu}_{l^{n}}^{\otimes(-w)}\right)=H^{2}\left(\bar{V}_{T}, \boldsymbol{\mu}_{l^{n}}^{\otimes(-w)}\right),
$$

which is inverse to the canonical map of $H^{2}\left(\overline{V_{T}^{\sigma(s)}}, \boldsymbol{\mu}_{l^{n}}^{\otimes(-w)}\right)$ to $H^{2}\left(\bar{V}_{T}, \boldsymbol{\mu}_{l^{n}}^{\otimes(-w)}\right)$. Recalling the definition of the operator $\mathfrak{I}^{*}(T s T)_{n}((2.6 .6))$, we have, by the functoriality of the cup product pairing, 


$$
\left(\mathfrak{I} *(T s T)_{n} x\right) \cup\left(\mathfrak{T} *(T s T)_{n} y\right)=t(s)_{n}(x \cup y)
$$

for all $x, y \in H^{1}\left(\bar{V}_{S}, \bar{F}_{n}\right)$. But since we assumed that $q$ is prime to $l L(S)$, we may take the representatives $\left\{s_{1}, \cdots, s_{d}\right\}$ of $S /\left(S \cap F^{\times}\right) T$ so that all the ${ }^{-}$-components of $s_{i}$ are 1 for all the primes $\mathfrak{l}$ above $l$. This shows that the isomorphism $\varepsilon_{n}$ in (4.5.7) is the base change of the identity (from Spec(Q)). Since the isomorphism $H^{2}\left(\bar{V}_{T}, \boldsymbol{\mu}_{l^{n}}\right) \cong \operatorname{Pic}\left(\bar{V}_{T}\right) / l^{n} \operatorname{Pic}\left(\bar{V}_{T}\right) \cong\left(Z / l^{n} Z\right)^{\left[k_{T}: F^{]}\right]}$is canonical, we conclude that $\operatorname{sum}\left(t(s)_{n}(x \cup y)\right)=\operatorname{sum}(x \cup y)$ with the notation of (4.2.4). Taking $\lim$ and tensoring $\boldsymbol{Q}_{l}$, we obtain our assertion.

Now for any non-zero $x \in W_{S, \rho}$, there exists an element $y \in W_{T, \rho}$ such that $(x, y)_{l} \neq 0$. Hence we have $\left(x, \sum_{i=1}^{d} \mathfrak{I}_{l}\left(T s_{i} T\right) y\right)_{l}=\left(\sum_{i=1}^{d} \mathfrak{I}_{l}\left(T s_{i}^{-1} T\right) x, y\right)_{l}=$ $d(x, y)_{l} \neq 0$, which completes the proof.

Q.E.D.

\section{$\S 5$. Construction of $\rho$}

In previous sections, we discussed the spaces $\mathfrak{S}(S, \rho)$ and the related $l$-adic representations starting from a representation $\rho$ satisfying certain conditions. The aim of this final section is to construct such representations ((5.4.3)). Our result here is a generalization of a result in Shimura [19] (cf. also [17] § 8).

5.1. Let us fix a finite Galois extension $K$ of $\boldsymbol{Q}$ contains $F$ and splits $B$ over $Q$; i.e. $B \otimes_{Q} K \cong M_{2}(K)^{g}$. We fix an isomorphism $\lambda$ of $B \otimes_{Q} K$ onto $M_{2}(K)^{g}$. $\lambda$ may be regarded as (the map on $K$-valued points induced by) an isomorphism of algebraic varieties over $K$. For $\sigma \in G=\operatorname{Gal}(K / Q)$, let $\lambda^{\sigma}$ be the conjugate of $\lambda$ by $\sigma$ in the sense of algebraic geometry: $\lambda^{\sigma}\left(a \otimes \alpha^{\sigma}\right)=$ $(\lambda(a \otimes \alpha))^{\sigma}$ for all $a \in B$ and $\alpha \in K$. Since $\lambda^{\sigma}$ is also a $K$-linear isomorphism of $B \otimes_{Q} K$ onto $M_{2}(K)^{g}$, we have $\lambda^{\sigma}=a_{\sigma} \circ \lambda$ with $a_{\sigma} \in$ Aut $_{K}\left(M_{2}(K)^{g}\right)$, the group of $K$-algebra automorphisms of $M_{2}(K)^{g}$. For an element $\Sigma \in \operatorname{Aut}_{K}\left(M_{2}(K)^{g}\right)$, we can find $s \in \mathbb{S}_{g}$, the symmetric group of degree $g$, and $X_{i} \in P G L_{2}(K)(1 \leqslant i \leqslant g)$ such that $\left(A_{1}, \cdots, A_{g}\right)^{\Sigma}=\left(X_{1}^{-1} A_{s-1(1)} X_{1}, \cdots, X_{g}^{-1} A_{s^{-1}(g)} X_{g}\right)$ for all $\left(A_{1}, \cdots, A_{g}\right)$ $\in M_{2}(K)^{g}$. We then write $\Sigma=\left(s ; X_{1}, \cdots, X_{g}\right)$. For $\Sigma=\left(s ; X_{1}, \cdots, X_{g}\right) \in$ Aut $_{K}$ $\left(M_{2}(K)^{g}\right)$ and $\sigma \in G$, we put $\Sigma^{\sigma}=\left(s ; X_{1}^{\sigma}, \cdots, X_{g}^{\sigma}\right)$. Then we easily see that

$$
a_{\tau} \circ a_{\sigma}^{\tau}=a_{\sigma \tau}
$$

for all $\sigma, \tau \in G$. Here and henceforth, we let $\operatorname{Aut}_{K}\left(M_{2}(K)^{g}\right)$ (resp. $\widetilde{S}_{g}$ ) act on $M_{2}(K)^{g}$ (resp. $\{1, \cdots, g\}$ ) from the right.

Let $\left\{\tau_{1}, \cdots, \tau_{g}\right\}$ be the set of all the different embeddings of $F$ into $C$. We extend each $\tau_{i}$ to an automorphism of $K$, and denote it by $\tau_{i}$ also. Let $\mathfrak{g}$ be the subgroup of $G$ corresponding to $F$. Then the right regular representation defines a homomorphism: 


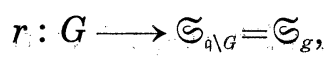

where we identify $g \mid G=\left\{g \tau_{1}, \cdots, g \tau_{g}\right\}$ with the index set $\{1, \cdots, g\}$ (in that order). For simplicity, the image of $\sigma \in G$ by $r$ is also denoted by $\sigma$ in the following. It is easy to see that $a_{\sigma}=\left(\sigma^{-1} ; X_{1}(\sigma), \cdots, X_{g}(\sigma)\right)$ with $X_{i}(\sigma) \in P G L_{2}$ $(K)$ under this convention.

5.2. Take non-negative integers $n_{i}(1 \leqslant i \leqslant g)$ and an integer $w$ satisfying

$$
n_{1} \equiv n_{2} \equiv \cdots \equiv n_{g} \equiv w \quad(\bmod 2) .
$$

Fixing such $\left\{n_{i}\right\}$ and $w$, we define a representation $\varphi^{\prime}$ of $G L_{2}(K)^{g}$ into $G L_{k}(K)$, with $\kappa=\prod_{i=1}^{g}\left(n_{i}+1\right)$, by

$$
\varphi^{\prime}\left(\left(A_{1} \cdot \cdots, A_{g}\right)\right)=\rho_{n_{1}}\left(A_{1}\right) \otimes \cdots \otimes \rho_{n_{g}}\left(A_{g}\right) \prod_{i=1}^{g} \operatorname{det}\left(A_{i}\right)^{\left(w-n_{i}\right) / 2}
$$

for all $\left(A_{1}, \cdots, A_{g}\right) \in G L_{2}(K)^{g}$, where $\rho_{n}$ denotes, as before, the symmetric tensor representation of degree $n$. We also define a representation $\varphi$ of $\left(B \otimes_{Q} K\right)^{\times}$into $G L_{k}(K)$ as the composite of $\varphi^{\prime}$ and $\lambda ; \varphi=\varphi^{\prime} \circ \lambda . \quad \varphi^{\prime}$ is obviously defined over $\boldsymbol{Q}$, and hence for any $\sigma \in G$, we have $\varphi^{\sigma}=\varphi^{\prime} \circ a_{\sigma} \circ \lambda$. We note that, for $\left(A_{1}, \cdots, A_{g}\right) \in G L_{2}(K)^{g}$,

$$
\begin{aligned}
\left(\varphi^{\prime} \circ a_{\sigma}\right)\left(\left(A_{1}, \cdots, A_{g}\right)\right)= & \rho_{n_{1}}\left(X_{1}(\sigma)^{-1} A_{\sigma(1)} X_{1}(\sigma)\right) \otimes \cdots \\
& \otimes \rho_{n_{g}}\left(X_{g}(\sigma)^{-1} A_{\sigma(g)} X_{g}(\sigma)\right) \prod_{i=1}^{g} \operatorname{det}\left(A_{\sigma(i)}\right)^{\left(w-n_{i}\right) / 2} .
\end{aligned}
$$

Through the map $r$, we may let $G$ act on $Z^{g}$ by the formula: $\left(\alpha_{1}, \cdots, \alpha_{g}\right)^{\sigma}$ $=\left(\alpha_{\sigma-1(1)}, \cdots, \alpha_{\sigma-1(g)}\right)$. Put

$$
H=\left\{\sigma \in G \mid\left(n_{1}, \cdots, n_{g}\right)^{\sigma}=\left(n_{1}, \cdots, n_{g}\right)\right\},
$$

and let $L$ be the subfield of $K$ corresponding to $H$.

For example, if $n_{1}=\cdots=n_{g}$, we have $L=\boldsymbol{Q}$. If all the $n_{i}$ are different, $L$ is the Galois closure of $F$ over $\boldsymbol{Q}$. In general, $L$ is intermediate to these fields, and independent of the choice of $K$.

Lemma (5.2.5). The notation being as above, for $\sigma, \tau \in G$, the following assertions are equivalent:

(i ) $\varphi^{\circ}$ and $\varphi^{\tau}$ are equivalent over $K$,

(ii) $\varphi^{\sigma}$ and $\varphi^{\tau}$ are equivalent over $C$,

(iii) $H \sigma=H \tau$.

Proof. Suppose that (ii) holds. Then the traces of $\varphi^{\sigma}$ and $\varphi^{\tau}$ must coincide. On the other hand, the matrix coefficients of $\rho_{n}(A)$ are homogene- 
ous polynomials of degree $n$ with respect to the coefficients of $A \in G L_{2}(K)$. By (5.2.3), we conclude that $\sigma^{-1}(\tau(i))=\left(\tau \sigma^{-1}\right)(i)=j$ implies $n_{i}=n_{j}$; i.e. (iii) holds. The implication (iii) $\Rightarrow$ (i) easily follows from (5.2.3). Q.E.D.

Explicitly, the equivalence between $\varphi$ and $\varphi^{\sigma}(\sigma \in H)$ can be written down as follows. Let $V_{i} \cong K^{n_{i+1}}$ be the representation space of $\rho_{n_{i}}$, and put $V=V_{1}$ $\otimes \cdots \otimes V_{g} \cong K^{k}$. For $\sigma \in H$, we define an automorphism $P(\sigma)$ of $V$ by

$$
P(\sigma)\left(v_{1} \otimes \cdots \otimes v_{g}\right)=\rho_{n_{1}}\left(X_{\sigma-1(1)}(\sigma)\right) v_{\sigma-1(1)} \otimes \cdots \otimes \rho_{n_{g}}\left(X_{\sigma-1(g)}(\sigma)\right) v_{\sigma-1(g)}
$$

for all $v_{1} \otimes \ldots \otimes v_{g} \in V$, where we choose a representative in $G L_{2}(K)$ of each $X_{i}(\sigma)$ and denote it also by the same letter. This is in fact well-defined since $\sigma \in H$. An easy computation shows that

$$
\varphi^{\sigma}=P(\sigma)^{-1} \varphi P(\sigma)=p(\sigma) \circ \varphi
$$

for $\sigma \in H$, where $p(\sigma)=\operatorname{Int}(P(\sigma))$, the inner automorphism defined by $P(\sigma)$. For $\sigma, \tau \in H$, the above relation implies that $p(\sigma \tau) \circ \varphi=p(\sigma)^{\tau} \circ p(\tau) \circ \varphi$ on $\left(B \otimes_{Q} K\right)^{\times}$, where $p(\sigma)^{\tau}=\operatorname{Int}\left(P(\sigma)^{\tau}\right)$. Since $\varphi$ is absolutely irreducible, we conclude that

$$
p(\sigma \tau)=p(\sigma)^{\tau} \circ p(\tau)
$$

i.e. $\{p(\sigma)\}$ satisfies the 1-cocycle condition. Put

$$
Y=\left\{x \in M_{\kappa}(K) \mid p(\sigma) x=x^{\sigma} \quad \text { for all } \sigma \in H\right\} .
$$

$Y$ is a central simple algebra over $L$ which is obtained by descending $M_{x}(K)$ to $L$ by the above 1-cocycle. (5.2.7) implies that $\varphi$ also descends to an $L$ rational homomorphism

$$
\psi:(B \underset{Q}{\otimes} L)^{\times} \longrightarrow Y^{\times}
$$

whose base change to $K$ is $\varphi$.

5.3. We recall that (cf. [18] 8.2), for a non-negative integer $n$, there is a matrix $\Theta_{n} \in G L_{n+1}(\boldsymbol{Q})$ such that

$$
\begin{aligned}
& { }^{t} \rho_{n}(\alpha) \Theta_{n} \rho_{n}(\alpha)=\operatorname{det}(\alpha)^{n} \Theta_{n} \quad \text { for all } \alpha \in G L_{2}(K), \\
& { }^{t} \Theta_{n}=(-1)^{n} \Theta_{n} .
\end{aligned}
$$

We define an inner product $(,)^{\prime}$ on $V=V_{1} \otimes \cdots \otimes V_{g}$ by

$$
\left(x_{1} \otimes \cdots \otimes x_{g}, y_{1} \otimes \cdots \otimes y_{g}\right)^{\prime}=\prod_{i=1}^{g}{ }^{t} x_{i} \Theta_{n_{i}} y_{i}
$$

for $x_{i}, y_{i} \in V_{i}$. For $\alpha \in\left(B \otimes_{Q} K\right)^{\times}$, we easily obtain 


$$
(\varphi(\alpha) x, y)^{\prime}=\left(x, \varphi\left(\alpha^{\prime}\right) y\right)^{\prime}
$$

for all $x, y \in V$ by (5.3.1), where $\iota=($ the canonical involution of $B) \otimes_{Q} 1_{K}$. Let $I^{\prime}$ be the $K$-rational anti-automorphism of $\operatorname{End}(V) \cong M_{x}(K)$ obtained as the adjoint with respect to $(,)^{\prime}$. By (5.3.2), $I^{\prime}$ is in fact an involution. (5.3.4) says that $I^{\prime} \circ \varphi(\alpha)=\varphi\left(\alpha^{c}\right)$. For $\sigma \in H$, take the conjugate of this equality; $I^{\prime \sigma}$ 。 $\varphi^{\sigma}\left(\alpha^{\sigma}\right)=\varphi^{\sigma}\left(\alpha^{\prime \sigma}\right)=\varphi^{\sigma}\left(\alpha^{\sigma}\right)$. This shows that $I^{\prime \sigma} \circ p(\sigma) \circ \varphi=p(\sigma) \circ I^{\prime} \circ \varphi$ on $\left(B \otimes_{Q} K\right)^{\times}$. Since $\varphi$ is absolutely irreducible, we conclude that $I^{\prime \sigma} \circ p(\sigma)=p(\sigma) \circ I^{\prime}$ on $M_{\kappa}(K)$. Therefore we obtain an $L$-rational involution $I$ of $Y$ whose base change to $K$ is $I^{\prime}$.

Since $Y$ has an involution, either one of the following cases occur:

(I) $Y \cong M_{\kappa}(L)$,

(II) $Y \cong M_{\kappa / 2}(D)$ with a division quaternion algebra $D$ over $L$.

We note that (II) occurs only when $n_{i} \not \equiv 0(\bmod 2)$. In the case (II), we fix an isomorphism $D \cong L^{4}$ as $L$-vector spaces, and hence an embedding $e: M_{\kappa / 2}(D)$ $\longrightarrow M_{2 n}(L)$. Combining $\psi$ with this map, we obtain a homomorphism

$$
\psi_{0}:\left(B \otimes_{Q} L\right)^{\times} \longrightarrow G L_{2 \kappa}(L)
$$

in the case (II). In the case (I), we put $\psi_{0}=\psi$. Put $\varepsilon=1$ (resp. $\left.\varepsilon=2\right)$ in the case (I) (resp. (II)).

Proposition (5.3.6). The notation being as above, suppose that $w \geqslant n_{i}$ $(1 \leqslant i \leqslant g)$. Then the representation $\psi_{0}$ has the following properties:

(5.3.6.1) $\psi_{0}$ is an L-rational homomorphism of algebraic groups over $L$.

(5.3.6.2) For $c \in\left(F \otimes_{Q} L\right)^{\times}$, we have $\psi_{0}(c)=N_{F \otimes_{Q} L / L}(c)^{w}$.

(5.3.6.3) For a maximal order $\mathfrak{o}$ of $B$, there is an $\mathfrak{r}_{L^{-}}$-lattice $\mathfrak{X}$ in $L^{\varepsilon \kappa}$ which is $\psi_{0}\left(\mathfrak{D} \cap B^{\times}\right)$-stable, and which satisfies $\left(\psi_{0}(\alpha)-\psi_{0}(1)\right) \mathfrak{X} \subseteq a \mathfrak{X}$ for any positive integer $a$ and any $\alpha \in \mathfrak{o} \cap B^{\times}$such that $\alpha-1 \in a \mathfrak{o}$.

(5.3.6.4) There is a non-degenerate L-bilinear form (, ) on $L^{\varepsilon k}$ which satisfies $\left(\psi_{0}(\alpha) x, \psi_{0}(\alpha) y\right)=N_{F \otimes_{Q} L / L}(\nu(\alpha))^{w}(x, y)$ for all $x, y \in L^{s i}$ and $\alpha \in\left(B \otimes_{Q} L\right)^{\times}$, where $\nu(\alpha)=\alpha \alpha^{\prime}$.

Here, $N_{F \otimes_{Q} L / L}$ denotes the reduced norm of $F \otimes_{Q} L$ over $L$.

Proof. The first assertion is obvious. The second assertion follows easily from the definition (5.2.2) of $\varphi^{\prime}$. To prove the third assertion, take a maximal order $R=\mathfrak{o}_{1} \oplus \cdots \oplus \mathfrak{o}_{g}$ of $M_{2}(K)^{g}$ which contains $\lambda(\mathfrak{o})$, where $\mathfrak{o}_{i}$ are maximal orders of $M_{2}(K)$. For each $i$, take an $\mathfrak{o}_{i}$-stable $\mathfrak{x}_{K}$-lattice $L_{i}$ in $K^{2}$, and let $\mathfrak{\Omega}_{i} \subseteq V_{i}$ be the symmetric tensors of degree $n_{i}$ with components in $L_{i}$. We also put $\mathfrak{Z}=\mathfrak{R}_{1} \otimes \cdots \otimes \mathfrak{R}_{g} \subseteq V$. In the case (I), it is easy to see that $\mathfrak{X}=\mathbb{R}$ $\cap L^{\kappa}$ satisfies (5.3.6.3). In the case (II), $Y \cong M_{\kappa / 2}(D)$ acts on $V \cong K^{k}$ via the natural inclusion $Y \subset Y \otimes_{L} K \cong M_{x}(K)$. Put $\mathscr{D}=\{\alpha \in Y \mid \alpha \Omega \subseteq \Omega\}$, and take $\mathfrak{X}$ to be an $\mathfrak{D}$-stable $\mathfrak{r}_{L}$-lattice in $L^{2 \kappa} \cong D^{\kappa / 2}$. Then this $\mathfrak{X}$ satisfies (5.3.6.3). 
To prove the last assertion, we first assume that we are in the case (I). Then there is a matrix $\Lambda \in G L_{\kappa}(L)$ such that $I(A)=\Lambda^{-1 t} A \Lambda$ for all $A \in M_{x}(L)$. The $L$-bilinear form defined by $(x, y)={ }^{t} x \Lambda y$ satisfies (5.3.6.4) by virtue of (5.3.4). In the case (II), let ' be the canonical involution of $D$ over $L$, and define the involution * of $M_{k / 2}(D)$ by $A^{*}={ }^{t} A^{\prime}={ }^{t}\left(a_{i j}^{\prime}\right)$ for $A=\left(a_{i j}\right) \in M_{k / 2}(D)$. We can find a matrix $\Lambda \in G L_{\kappa / 2}(D)$ such that $I(A)=\Lambda^{-1} A^{*} \Lambda$ for all $A \in M_{\kappa / 2}(D)$. Recall that we fixed an $L$-linear isomorphism of $D^{x / 2}$ onto $L^{2 k}$ which we now call $j$. For $d={ }^{t}\left(d_{1}, \cdots, d_{\kappa / 2}\right) \in D^{k / 2}$, put $d^{\prime}={ }^{t}\left(d_{1}^{\prime}, \cdots, d_{\kappa / 2}^{\prime}\right)$. We then put $(j(x), j(y))=\operatorname{tr}_{D / L}\left({ }^{t} x^{\prime} \Lambda y\right)$ for $x, y \in D^{\kappa / 2}$, where $\operatorname{tr}_{D / L}$ denotes the reduced trace of $D$ over $L$. It is easy to see that this $L$-bilinear form on $L^{2 \kappa}$ satisfies (5.3.6.4).

Q.E.D.

5.4. Before constructing $\rho$, we recall some facts about the restriction of scalar functor (Weil [20] 1.3). In general, let $K / k$ be a finite separable extension of fields, and suppose that a $K$-variety $V$ is given. By definition, the $k$-variety $R_{K / k}(V)$ (or $\prod_{K / k} V$ in the terminology of Grothendieck), if exists, represents the functor $T \mapsto \operatorname{Hom}_{K}\left(T \otimes_{k} K, V\right)$ for $k$-schemes $T . \quad R_{K / k}$ $(V)$ does exist when $V$ is quasi-projective over $K$, and we hereafter assume that this is the case. Consider the special case when $V=V_{0} \otimes_{k} K$ with a $k$ variety $V_{0}$. In this case, we have the natural map:

$$
\operatorname{Hom}_{k}\left(T, V_{0}\right) \longrightarrow \operatorname{Hom}_{k}(T \underset{k}{\otimes} K, V)=\operatorname{Hom}_{k}\left(T, R_{K / k}(V)\right)
$$

for $k$-schemes $T$, where the left map is the base change. When $T=V_{0}$, there corresponds to the identity morphism in the left a $k$-morphism $f$ of $V_{0}$ to $R_{K / k}(V)$. On the other hand, by the construction of $R_{K / k}(V)$ ([20] loc. cit.), $R_{K / k}(V) \otimes_{k} K$ is canonically isomorphic to the product of $d=[K: k]$ copies of $V$ over $K$. It is easy to see that the base change of $f$ to $K$ can be identified with the diagonal morphism: $V \rightarrow V \times \cdots \times V$ (product of $d$ copies). Also, it is tautological that, for any $k$-algebra $A$, the map $f(A): V_{0}(A) \rightarrow R_{K / k}(V)(A)$ $=V_{0}\left(A \otimes_{k} K\right)$ is the map induced by the natural map of $A$ into $A \otimes_{k} K$.

We had an $L$-rational representation $\psi_{0}$. Apply $R_{L / Q}$ to $\psi_{0}$ to obtain a $\boldsymbol{Q}$-rational homomorphism $R_{L / Q}\left(\psi_{0}\right): R_{L / Q}\left(\left(B \otimes_{Q} L\right)^{\times}\right) \rightarrow R_{L / Q}\left(G L_{\varepsilon x}(L)\right)$, where, as before, we consider $\left(B \otimes_{Q} L\right)^{\times}$and $G L_{\varepsilon x}(L)$ as algebraic groups over $L$. By the above remark, we have a homomorphism $B^{\times} \rightarrow R_{L / Q}\left(\left(B \otimes_{Q} L\right)^{\times}\right)$. On the other hand, for any $Q$-algebra $A$, we have $R_{L / Q}\left(G L_{\varepsilon x}(L)\right)(A)=G L_{\varepsilon k}\left(A \otimes_{Q} L\right)$. Fixing a $\boldsymbol{Q}$-linear isomorphism of $L$ onto $\boldsymbol{Q}^{d}$ with $d=[L: Q]$, we have a natural homomorphism $G L_{\varepsilon x}\left(A \otimes_{Q} L\right) \rightarrow G L_{\varepsilon x d}(A)$ which is functorial in $A$. We have thus obtained a $\boldsymbol{Q}$-rational homomorphism $R_{L / Q}\left(G L_{\varepsilon x}(L)\right) \rightarrow G L_{\varepsilon n d}(\boldsymbol{Q})$. Combining these three homomorphisms, we obtain

$$
\rho: B^{\times} \longrightarrow G L_{\varepsilon \alpha d}(\boldsymbol{Q}) .
$$


TheOREM (5.4.3). Suppose that $w \geqslant n_{i}(1 \leqslant i \leqslant g)$. Then the above $\rho$ satisfies the conditions (1.1.1), (1.1.2), (2.1.6) and (4.1.1). In the case (I), the map on $K$-rational points $\rho_{K}$ is equivalent over $K$ to the direct sum $\oplus_{\sigma \in H \backslash G} \varphi^{\sigma}$. In the case (II), $\rho_{K}$ is equivalent over $K$ to the direct sum of two copies of $\oplus_{\sigma \in H \backslash G} \varphi^{\sigma}$.

Proof. The condition (1.1.1) is obviously satisfied. Take $\boldsymbol{Q}$-rational points of $\rho$ :

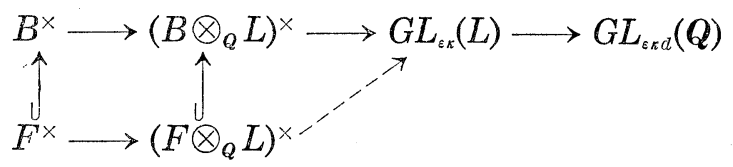

By the remark at the beginning of this subsection and (5.3.6.2), we see that (1.1.2) is satisfied. In (5.3.6.3), we have shown the existence of an $\mathfrak{r}_{L}$-lattice $\mathfrak{X}$ in $L^{\varepsilon r}$ satisfying the condition similar to (2.1.6). Viewing this $\mathfrak{X}$ as a $Z$ lattice in $L^{\varepsilon x} \cong Q^{e x d}$, which we call $X$, we see easily that this $X$ satisfies (2.1.6). Next we define the $\boldsymbol{Q}$-bilinear form on $L^{\varepsilon \kappa} \cong Q^{\varepsilon \kappa d}$ by $\operatorname{Tr}_{L / Q}((x, y))$ for $x, y \in L^{\varepsilon \kappa}$, where (, $)$ is as in (5.3.6.4). This obviously satisfies (4.1.1).

To prove the last assertion, we assume that we are in the case (II). Take $K$-rational points of $\rho$ :

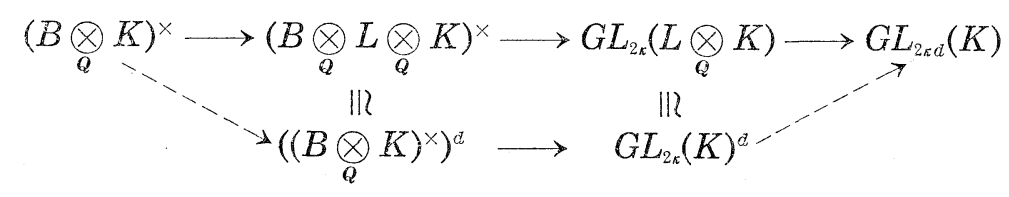

where the vertical isomorphisms are induced by the isomorphism $L \otimes_{Q} K \cong$ $K^{d}$, and the lower maps are defined by the commutativity of the diagram. The left dotted map is the diagonal map by the preceding remark. The lower horizontal map is (the base change to $K$ of) $\oplus_{\sigma \in H \backslash G} \psi_{0}^{\sigma}$ by the construction of $R_{L / Q}$ ([20] loc. cit.). Since $\psi_{0}$ factors as

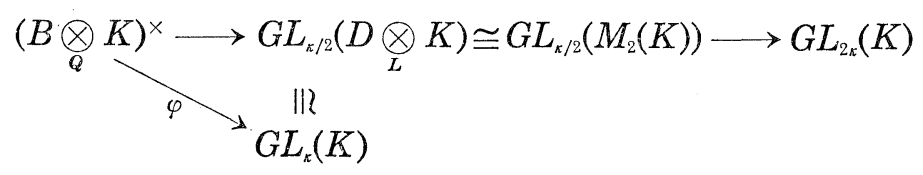

over $K$, where the right map is induced by the isomorphism $M_{2}(K) \cong K^{4}$, we obtain our assertion immediately. The proof in the case (I) is similar and simpler.

Q.E.D.

REMARK (5.4.4). By the construction, without assuming that $w \geqslant n_{i}$ $(1 \leqslant i \leqslant g)$, we still obtain a representation $\rho$ satisfying (1.1.1), (1.1.2) and (4.1.1). 
We close this paper by explicitly writing down the consequence of (4.3.1) and (5.4.3). For non-negative integers $k_{i}(1 \leqslant i \leqslant g)$ and an integer $w$, let $\Psi$ be the representation over $C$ of $B_{\infty_{+}+} \cong G L_{2}^{+}(R) \times\left(H^{\times}\right)^{g-1}$ defined by $G L_{2}^{+}(\boldsymbol{R}) \times\left(\boldsymbol{H}^{\times}\right)^{g-1} \ni\left(x_{1}, \cdots, x_{g}\right) \mapsto \rho_{k_{2}}\left(x_{2}\right) \otimes \cdots \otimes \rho_{k_{g}}\left(x_{g}\right) \operatorname{det}\left(x_{1}\right)^{w / 2} \prod_{i=2}^{g} \operatorname{det}\left(x_{i}\right)^{\left(w-k_{i}\right) / 2}$, where we consider $\boldsymbol{H}^{\times}$as a subgroup of $\left(\boldsymbol{H} \otimes_{R} C\right)^{\times} \cong G L_{2}(C)$. We can then define the space $\widetilde{S}_{k_{1+2}}(\Gamma, \Psi)$ as in 1.3 , which we write $\subseteq\left(\Gamma ;\left(k_{1}, \cdots, k_{g}\right) ; w\right)$. We also define $\subseteq\left(S ;\left(k_{1}, \cdots, k_{g}\right) ; w\right)$ as the direct sum $\oplus_{i=1}^{h} \subseteq\left(\Gamma_{S_{i}} ;\left(k_{1}, \cdots, k_{g}\right)\right.$; $w)$ for $S \in \mathscr{Z}$, with the notation as in 1.4.

Fix non-negative integers $n_{i}(1 \leqslant i \leqslant g)$ and an integer $w$ satisfying (5.2.1), and let $\rho$ be the representation constructed by the above procedure from $\left\{n_{i}\right\}$ and $w$. We then have

$$
\begin{aligned}
& \Im(S, \rho)=\bigoplus_{\sigma \in H \backslash G} \subseteq\left(S ;\left(n_{1}, \cdots, n_{g}\right)^{\sigma} ; w\right) \text { in the case (I), } \\
& \Im(S, \rho)=\bigoplus_{\sigma \in H \backslash G}^{2} \bigoplus_{\sigma \in G} \widetilde{S}\left(S ;\left(n_{1}, \cdots, n_{g}\right)^{\sigma} ; w\right) \text { in the case (II). }
\end{aligned}
$$

Theorem (5.4.6). The notation being as above, let $S$ be a member of $\mathscr{Z}$ which is stable under the canonical involution. Then, for each rational prime $l$, there exists an l-adic representation

$$
\psi_{S, \rho}: \operatorname{Gal}(\bar{F} / F) \longrightarrow G L_{2 \operatorname{dim}_{C} \widetilde{S}(S, \rho)}\left(\boldsymbol{Q}_{l}\right)
$$

which has the properties (4.3.1.1) and (4.3.1.2).

Proof. If $w \geqslant n_{i}(1 \leqslant i \leqslant g)$, this follows from (4.3.1) and (5.4.3). In the general case, $\rho^{\prime}=\rho \otimes N_{F / Q}(\nu())^{a}$ with a suitable positive integer $a$ satisfies that condition, and hence $\psi_{S, \rho^{\prime}}$ exists. Let $W_{S, \rho^{\prime}}$ be the representation space of $\psi_{S, \rho^{\prime}}$, and define $\psi_{S, \rho}$ as the natural representation of $\operatorname{Gal}(\bar{F} / F)$ on the Tate twist $W_{S, \rho^{\prime}}(a)$. Then we have

$$
\operatorname{det}\left(1-\psi_{S, \rho^{\prime}}\left(\sigma_{\mathfrak{p}}\right) T\right)=\operatorname{det}\left(1-\psi_{S, \rho}\left(\sigma_{\mathfrak{p}}\right) N_{F / Q}(\mathfrak{p})^{a} T\right)
$$

for all $\mathfrak{p} \times l D(B / F) L(S)$, where $\sigma_{\mathfrak{p}}$ is a Frobenius element at $\mathfrak{p}$. Our assertion then follows from (1.5.8).

Q.E.D.

\section{References}

[1] M. Artin, A. Grothendieck and J. L. Verdier, Théorie des topos et cohomologie étale des schémas (SGA 4), Lecture Notes in Math., vol. 269, 270, 305, Springer, 1972-1973.

[2] C. Chevalley, Deux théorèmes d'arithmétique, J. Math. Soc. Japan, 3 (1951), 36-44.

[ 3 ] P. Deligne, Formes modulaires et représentations $l$-adiques, Sém. Bourbaki, exp. 355,1969 . 
[ 4 ] P. Deligne, La conjecture de Weil I, Publ. Math. IHES, 43 (1974), 273-307.

[5] M. Eichler, Quaternäre quadratische Formen und die Riemannsche Vermutung für die Kongruenzzetafunktion, Arch. Math., 5 (1954), 355-366.

[6] Y. Ihara, Hecke polynomials as congruence $\zeta$ functions in elliptic modular case, Ann. of Math., 85 (1967), 267-295.

[ 7 ] Y. Ihara, Congruence relations and Shimura curves, Proc. of Symposia in Pure Math., vol. 33 (1979), Part 2, 291-311.

[ 8 ] M. Kuga and G. Shimura, On the zeta function of a fibre variety whose fibres are abelian varieties, Ann. of Math., 82 (1965), 478-539.

[ 9 ] E. Lamprecht, Restabbildung von Divisoren, Arch. Math., 8 (1957), 255-265.

[10] R. P. Langlands, Modular forms and $l$-adic representations, in Lecture Notes in Math., vol. 349, Springer, 1973, 361-500.

[11] R. P. Langlands, On the zeta-functions of some simple Shimura varieties, Canad. J. of Math., XXXI-6 (1979), 1121-1216.

[12] Y. Matsushima and G. Shimura, On the cohomology groups attached to certain vector valued differential forms on the product of the upper half planes, Ann. of Math., 78 (1963), 417-449.

[13] Y. Morita, Reduction modulo $\mathfrak{乛}$ of Shimura curves, Hokkaido Math. J., X-2 (1981), 209-238.

[14] J.-P. Serre, Cohomologie des groupes discrets, Ann. of Math. Studies 70, Princeton Univ. Press, 1971, 77-169.

[15] G. Shimura, On Dirichlet series and abelian varieties attached to automorphic forms, Ann. of Math., 76 (1962), 237-294.

[16] G. Shimura, Construction of class fields and zeta functions of algebraic curves, Ann. of Math., 85 (1967), 58-159.

[17] G. Shimura, On canonical models of arithmetic quotients of bounded symmetric domains, Ann. of Math., 91 (1970), 144-222.

[18] G. Shimura, Introduction to the arithmetic theory of automorphic functions, Publ. Math. Soc. Japan, Iwanami Shoten and Princeton Univ. Press, 1971.

[19] G. Shimura, An $l$-adic method in the theory of automorphic forms, (1968), unpublished.

[20] A. Weil, Adeles and algebraic groups, lecture notes, Institute for Advanced Study, Princeton, 1961.

Department of Mathematics

KYOTO UNIVERSITY

Кчото 606, JAPAN 